TRANSACTIONS OF THE

AMERICAN MATHEMATICAL SOCIETY

Volume 359, Number 7, July 2007, Pages 3025-3069

S 0002-9947(07)03988-8

Article electronically published on January 29, 2007

\title{
ALTERNATING FORMS AND THE BRAUER GROUP OF A GEOMETRIC FIELD
}

\author{
ERIC S. BRUSSEL
}

\begin{abstract}
We compute the theory of $H^{2}(G, \mathbb{Q} / \mathbb{Z})$ for any proabelian group $G$, using a natural isomorphism with the group $\operatorname{Alt}(G, \mathbb{Q} / \mathbb{Z})$ of continuous alternating forms. We use this to establish a sort of generic behavioral ideal, or role model, for the Brauer group $\operatorname{Br}(F)$ of a geometric field $F$ of characteristic zero. We show this ideal is attained in several interesting cases.
\end{abstract}

\section{INTRODUCTION}

Let $F$ be a geometric field of characteristic zero, with absolute Galois group $G_{F}$. Since $F$ contains an algebraically closed field, it contains the group $\mu$ of all roots of unity. Let $\mu_{n}$ denote the $n$-torsion subgroup of $\mu$, for $n \in \mathbb{N}$. Since $F$ contains $\mu_{n}$, there is a natural $\mathrm{G}_{F}$-isomorphism $\mu_{n}^{\otimes 2} \simeq \mu_{n}$, and the cup product, or Galois symbol, maps into the Brauer group,

$$
\mathrm{H}^{1}\left(\mathrm{G}_{F}, \mu_{n}\right) \otimes_{\mathbb{Z}} \mathrm{H}^{1}\left(\mathrm{G}_{F}, \mu_{n}\right) \longrightarrow \mathrm{H}^{2}\left(\mathrm{G}_{F}, \mu\right) \simeq \operatorname{Br}(F) .
$$

The natural map $F^{\times} \rightarrow \mathrm{H}^{1}\left(\mathrm{G}_{F}, \mu_{n}\right)$ is surjective by Hilbert 90 , and if $x$ and $y$ are elements of $F^{\times}$, the image of $x \otimes y$ under the induced pairing is a symbol $\left[(x, y)_{\omega_{n}}\right]$. The famous Merkurjev-Suslin theorem shows that these symbols generate $\operatorname{Br}(F)$, as $n$ ranges over the natural numbers. Let $G=\mathrm{G}_{F}^{\mathrm{ab}}$, the maximal abelian quotient of $\mathrm{G}_{F}$. We are interested in the bottom row of the commutative diagram

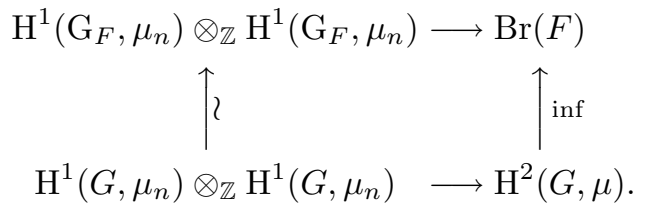

We show that $\mathrm{H}^{2}(G, \mu)$ is generated by cup products, a well known result (see TW. Remark 4.11]). We then develop a complete, computationally effective theory for the bottom row, for any proabelian group, using the theory of alternating forms. Since the inflation map is surjective and computationally transparent, we obtain implications for $\operatorname{Br}(F)$.

In the end we establish what we feel is a behavioral role model for the division algebras and Brauer group $\operatorname{Br}(F)$ of $F$. This consists of bounds for a given Brauer class's most important invariants, as well as explicit computations of the fundamental functorial maps with respect to abelian extensions. Our main tool is a natural isomorphism $\mathrm{H}^{2}(G, \mathbb{Q} / \mathbb{Z}) \simeq \operatorname{Alt}(G, \mathbb{Q} / \mathbb{Z})$, where $G$ is any proabelian group

Received by the editors September 16, 2003 and, in revised form, March 7, 2005.

2000 Mathematics Subject Classification. Primary 16K50; Secondary 20J06.

(C) 2007 American Mathematical Society Reverts to public domain 28 years from publication 
and $\operatorname{Alt}(G, \mathbb{Q} / \mathbb{Z}) \simeq \operatorname{Hom}\left(\bigwedge^{2} G, \mathbb{Q} / \mathbb{Z}\right)$ is the group of continuous alternating forms on $G$ with values in $\mathbb{Q} / \mathbb{Z}$. We compute the theory of $\operatorname{Alt}(G, \mathbb{Q} / \mathbb{Z})$ using simple linear algebra techniques, developed and applied over $\mathbb{Q} / \mathbb{Z}$. We define and compute the exponent, index, and canonical form of an alternating form, and we compute inflation, restriction, and corestriction maps. Letting $G$ be a Galois group over $F$, we then use the Galois symbol to compute the composite $\operatorname{Alt}(G, \mathbb{Q} / \mathbb{Z}) \rightarrow \mathrm{H}^{2}(G, \mathbb{Q} / \mathbb{Z}) \rightarrow \operatorname{Br}(F)$ in terms of symbol classes, converting our theory of alternating forms on $G$ into a theory of symbol classes in $\operatorname{Br}(F)$. When $G$ is $F$ 's absolute abelian Galois group, the map is an isomorphism. In this case $G$ is torsion-free, so it has a topological basis, and we can make explicit calculations, as we show in the example below.

In certain interesting cases our bounds are sharp, and we obtain explicit index formulas and a simple algorithm that determines a Brauer class's representative division algebra. This is the case when $F=\mathbb{C}\left(\left(x_{1}\right)\right) \cdots\left(\left(x_{n}\right)\right)$, the field of iterated power series, or when $F$ is a rational function field $F=k\left(x_{1}, \ldots, x_{n}\right)$ containing $\mathbb{C}$.

This paper arose from the author's work in [B] on the Brauer group of the field $F=\mathbb{C}\left(\left(x_{1}\right)\right) \cdots\left(\left(x_{n}\right)\right)$ (see also $[\mathrm{TW}]$ ). That paper was based on an ad hoc isomorphism between $\operatorname{Br}(F)$ and the group of alternating matrices $\operatorname{Alt}_{n}(\mathbb{Q} / \mathbb{Z})$. This paper explains the nature of that isomorphism, and extends it to its proper level of applicability.

Example. We show how to calculate index, representative division algebra, and restriction and corestriction in the Brauer group of the rational function field $F=\mathbb{C}\left(x_{1}, x_{2}, x_{3}, x_{4}\right)$. Our theory computes the part of the Brauer group that is expressible in terms of the $x_{i}$. This is a subgroup, which we call $\operatorname{Br}^{\circ}(\Omega / F)$, of the relative Brauer group $\operatorname{Br}(\Omega / F)$, where $\Omega$ is the (infinite) Kummer extension obtained by taking all possible roots of the $x_{i}$. Let $\left[\left(x_{i}, x_{j}\right)_{\omega_{d}}^{r}\right]$ denote the Brauer class of the $r$-th tensor power of the symbol algebra of degree $d$ defined by $x_{i}, x_{j}$, and a primitive root of unity $\omega_{d}$. Let $\boldsymbol{\alpha} \in \operatorname{Br}(F)$ be the element

$\boldsymbol{\alpha}=\left[\left(x_{1}, x_{2}\right)_{\omega_{4}}^{-1}\right]+\left[\left(x_{1}, x_{3}\right)_{\omega_{20}}^{9}\right]+\left[\left(x_{1}, x_{4}\right)_{\omega_{20}}^{-1}\right]+\left[\left(x_{2}, x_{3}\right)_{\omega_{20}}\right]+\left[\left(x_{2}, x_{4}\right)_{\omega_{5}}^{-1}\right]+\left[\left(x_{3}, x_{4}\right)_{\omega_{4}}\right]$.

$\boldsymbol{\alpha}$ 's coefficients define the alternating matrix

$$
\mathcal{A}=\left(\begin{array}{cccc}
0 & -\frac{1}{4} & \frac{9}{20} & -\frac{1}{20} \\
\frac{1}{4} & 0 & \frac{1}{20} & -\frac{1}{5} \\
-\frac{9}{20} & -\frac{1}{20} & 0 & \frac{1}{4} \\
\frac{1}{20} & \frac{1}{5} & -\frac{1}{4} & 0
\end{array}\right) \quad \in \operatorname{Alt}_{4}(\mathbb{Q} / \mathbb{Z})
$$

Although this matrix is associated directly to the given Brauer class, in our theory it is the matrix of an alternating form on the Galois group $\mathrm{G}_{\Omega / F} \simeq \widehat{\mathbb{Z}}^{4}$, given by specifying a basis of generators for $G$ corresponding to the elements $x_{i}$ via Kummer theory. The index of $\boldsymbol{\alpha}$ is given by the formula

$$
\begin{aligned}
\operatorname{ind}(\boldsymbol{\alpha}) & =\operatorname{lcm}\left\{\left|a_{i j}\right|,\left|a_{12} a_{34}-a_{13} a_{24}+a_{14} a_{23}\right|\right\} \\
& =\operatorname{lcm}\{|1 / 4|,|1 / 20|,|1 / 5|,|1 / 40|\}=40,
\end{aligned}
$$

computed by taking the least common multiples of the orders in $\mathbb{Q} / \mathbb{Z}$ of the subpfaffians of the alternating matrix $\mathcal{A}$. To find the division algebra underlying $\boldsymbol{\alpha}$ is simple: we "2-block-diagonalize" $\mathcal{A}$ using the standard algorithm. We understand 
this as a basis change on $G$, given by the diagonalizing matrix $\mathcal{P}$. In this case, if

$$
\mathcal{P}=\left(\begin{array}{cccc}
3 & 0 & -1 & 0 \\
-2 & 5 & 1 & 1 \\
5 & 8 & -2 & 2 \\
15 & 7 & -6 & 2
\end{array}\right) \quad \in \mathrm{GL}_{4}(\mathbb{Z})
$$

then we easily check

$$
\mathcal{A}^{\prime}=\mathcal{P}^{*} \mathcal{A P}=\left(\begin{array}{cccc}
0 & \frac{1}{2} & 0 & 0 \\
-\frac{1}{2} & 0 & 0 & 0 \\
0 & 0 & 0 & \frac{1}{20} \\
0 & 0 & -\frac{1}{20} & 0
\end{array}\right) \quad \in \operatorname{Alt}_{4}(\mathbb{Q} / \mathbb{Z})
$$

where $\mathcal{P}^{*}$ is $\mathcal{P}$ 's transpose. This shows $\boldsymbol{\alpha}$ has canonical form

$$
\boldsymbol{\alpha}=\left[\left(x_{1}^{\prime}, x_{2}^{\prime}\right)_{\omega_{2}}\right]+\left[\left(x_{3}^{\prime}, x_{4}^{\prime}\right)_{\omega_{20}}\right]
$$

with representative division algebra $D \simeq\left(x_{1}^{\prime}, x_{2}^{\prime}\right)_{\omega_{2}} \otimes\left(x_{3}^{\prime}, x_{4}^{\prime}\right)_{\omega_{20}}$. The $x_{i}^{\prime}$ are algebraically independent over $\mathbb{C}$, and $x_{i}^{\prime}=x_{1}^{q_{i 1}} x_{2}^{q_{i 2}} x_{3}^{q_{i 3}} x_{4}^{q_{i 4}}$, with exponents taken from the $i$-th row of the matrix

$$
\mathcal{Q}=\mathcal{P}^{-1}=\left(\begin{array}{cccc}
4 & -2 & 3 & -2 \\
-4 & 4 & -5 & 3 \\
11 & -6 & 9 & -6 \\
17 & -17 & 22 & -13
\end{array}\right)
$$

If the $x_{i}$ were not algebraically independent over $\mathbb{C}$, the canonical form would merely be a candidate for the underlying division algebra, and the number 40 an upper bound for ind $(\boldsymbol{\alpha})$.

Now let $K / F$ be a finite subextension of $\Omega / F$, defined by $K=F\left(y_{1}, y_{2}, y_{3}, y_{4}\right)$, where $y_{i}=x_{1}^{q_{i 1}^{\prime}} x_{2}^{q_{i 2}^{\prime}} x_{3}^{q_{i 3}^{\prime}} x_{4}^{q_{i 4}^{\prime}}$, and the $q_{i j}^{\prime}$ are given by the matrix

$$
Q^{\prime}=\left(q_{i j}^{\prime}\right)=\left(\begin{array}{cccc}
\frac{7}{15} & -\frac{2}{15} & -\frac{1}{5} & -\frac{7}{15} \\
-\frac{8}{15} & \frac{11}{30} & \frac{4}{5} & \frac{61}{30} \\
-\frac{2}{15} & -\frac{1}{30} & \frac{1}{5} & \frac{19}{30} \\
-\frac{2}{15} & -\frac{1}{30} & \frac{1}{5} & -\frac{11}{30}
\end{array}\right) \quad \in \mathrm{GL}_{4}(\mathbb{Q}) .
$$

It is important that the $y_{i}$ form a basis for the group $\left(K^{\times} / F^{\times}\right)_{\text {tor }}$, so that Q's inverse is defined over $\mathbb{Z}$. Here,

$$
\mathcal{P}^{\prime}=\left(\mathcal{Q}^{\prime}\right)^{-1}=\left(\begin{array}{cccc}
3 & 1 & -1 & 0 \\
0 & 2 & -7 & -1 \\
2 & 1 & 0 & 3 \\
0 & 0 & 1 & -1
\end{array}\right) \quad \in \mathrm{M}_{4}(\mathbb{Z})
$$

This is the matrix of generators for the closed subgroup of $G$ that fixes $K$. It follows that $[K: F]=\left|\operatorname{det}\left(\mathcal{P}^{\prime}\right)\right|=30$. Let $\mathcal{A} \in \operatorname{Alt}_{4}(\mathbb{Q} / \mathbb{Z})$ and $\boldsymbol{\alpha} \in \operatorname{Br}(F)$ be as above. The matrices $\mathcal{P}^{\prime}$ and $\mathcal{Q}^{\prime}$ are used to perform functorial operations on the alternating matrix $\mathcal{A}$ representing $\boldsymbol{\alpha}$. For example, $\boldsymbol{\beta}:=\operatorname{res}(\boldsymbol{\alpha}) \in \operatorname{Br}(K)$ is determined by the matrix

$$
\mathcal{B}=\mathcal{P}^{\prime *} \mathcal{A P}^{\prime}=\left(\begin{array}{cccc}
0 & -\frac{1}{4} & \frac{1}{5} & \frac{11}{20} \\
\frac{1}{4} & 0 & \frac{17}{20} & \frac{3}{20} \\
-\frac{1}{5} & -\frac{17}{20} & 0 & -\frac{1}{20} \\
-\frac{11}{20} & -\frac{3}{20} & \frac{1}{20} & 0
\end{array}\right) \quad \in \operatorname{Alt}_{4}(\mathbb{Q} / \mathbb{Z})
$$


Explicitly, reading off of the upper right triangle, we have

$$
\begin{aligned}
\boldsymbol{\beta}= & \operatorname{res}(\boldsymbol{\alpha})=\left[\left(y_{1}, y_{2}\right)_{\omega_{4}}^{-1}\right]+\left[\left(y_{1}, y_{3}\right)_{\omega_{5}}\right]+\left[\left(y_{1}, y_{4}\right)_{\omega_{20}}^{11}\right] \\
& +\left[\left(y_{2}, y_{3}\right)_{\omega_{20}}^{17}\right]+\left[\left(y_{2}, y_{4}\right)_{\omega_{20}}^{3}\right]+\left[\left(y_{3}, y_{4}\right)_{\omega_{20}}^{-1}\right],
\end{aligned}
$$

where each $y_{i}=\prod_{j} x_{j}^{q_{i j}^{\prime}}$ is given by $\mathcal{Q}^{\prime}$. The index of $\boldsymbol{\beta}$ is

$$
\begin{aligned}
\operatorname{ind}(\boldsymbol{\beta}) & =\operatorname{lcm}\{|1 / 4|,|1 / 5|,|1 / 20|,|1 / 80-3 / 100+187 / 400|\} \\
& =\operatorname{lcm}\{4,5,20,|9 / 20|\}=20 .
\end{aligned}
$$

Since the index of $\boldsymbol{\alpha}$ was 40 , we see that extending scalars to this field extension $K / F$ of degree 30 reduces the index of $\boldsymbol{\alpha}$ by a factor of two.

Conversely, suppose $\boldsymbol{\beta} \in \operatorname{Br}(K)$ is given by $(*)$. Then $\boldsymbol{\gamma}:=\operatorname{cor}(\boldsymbol{\beta}) \in \operatorname{Br}(F)$ is determined by the matrix

$$
\mathcal{C}=30\left(\mathcal{Q}^{\prime *} \mathcal{B Q}^{\prime}\right)=\left(\begin{array}{cccc}
0 & \frac{1}{2} & \frac{1}{2} & \frac{1}{2} \\
-\frac{1}{2} & 0 & \frac{1}{2} & 0 \\
-\frac{1}{2} & -\frac{1}{2} & 0 & \frac{1}{2} \\
-\frac{1}{2} & 0 & -\frac{1}{2} & 0
\end{array}\right) \quad \in \operatorname{Alt}_{4}(\mathbb{Q} / \mathbb{Z})
$$

Translating back to $\operatorname{Br}(F)$, we have

$$
\boldsymbol{\gamma}=\operatorname{cor}(\boldsymbol{\beta})=\left[\left(x_{1}, x_{2}\right)_{\omega_{2}}\right]+\left[\left(x_{1}, x_{3}\right)_{\omega_{2}}\right]+\left[\left(x_{1}, x_{4}\right)_{\omega_{2}}\right]+\left[\left(x_{2}, x_{3}\right)_{\omega_{2}}\right]+\left[\left(x_{3}, x_{4}\right)_{\omega_{2}}\right],
$$

where $x_{i}=\prod_{j} y_{j}^{p_{i j}^{\prime}}$ is given by $\mathcal{P}^{\prime}$. Note that $\gamma=\operatorname{cor} \cdot \operatorname{res}(\boldsymbol{\alpha})=30 \boldsymbol{\alpha}$, as required. The index is

$$
\operatorname{ind}(\gamma)=\operatorname{lcm}\{|1 / 2|,|1 / 4-0+1 / 4|\}=2 .
$$

Thus the corestriction reduces $\boldsymbol{\beta}$ 's index by a factor of 10 .

\section{Conventions}

Write $\mathcal{P}$ for the set of rational primes. If $d \in \mathbb{N}$, let $\operatorname{supp}(d)$ denote $d$ 's prime support. Use $\mathfrak{m}$ to denote a subset of $\mathcal{P}$. Let $\mathbb{N}(\mathfrak{m})$ denote the set of positive integers supported on $\mathfrak{m}$.

For each prime $p$, let $\mathbb{Z}_{p}$ be the additive group underlying the ring of $p$-adic integers. If $\mathfrak{m} \subset \mathcal{P}$ is a set of primes, let $\mathbb{Z}_{\mathfrak{m}}$ denote the product $\prod_{\mathfrak{m}} \mathbb{Z}_{p}$. Let $\widehat{\mathbb{Z}}$ be the profinite completion of $\mathbb{Z}$; then $\widehat{\mathbb{Z}}=\mathbb{Z}_{\mathfrak{m}}$, where $\mathfrak{m}$ is the set of all primes. Let $\mathbb{Z}[1 / p]$ denote the additive group of the localization of $\mathbb{Z}$ with respect to the prime number $p$, and let $\mathbb{Z}[1 / p] / \mathbb{Z}$ denote the quotient, a divisible torsion $p$-group. Let $\mathbb{Z}[1 / \mathfrak{m}] / \mathbb{Z}$ denote the (divisible) direct sum $\coprod_{\mathfrak{m}} \mathbb{Z}[1 / p] / \mathbb{Z}$. Note that $\mathbb{Z}[1 / \mathfrak{m}] / \mathbb{Z}=\mathbb{Q} / \mathbb{Z}$ when $\mathfrak{m}$ is the set of all primes.

Each $\mathbb{Z}_{\mathfrak{m}}$ acts diagonally on $\mathbb{Z}[1 / \mathfrak{m}] / \mathbb{Z}$ with respect to the primary decomposition. Explicitly, if $x=\prod_{\mathfrak{m}} x_{p} \in \mathbb{Z}_{\mathfrak{m}}$, where $x_{p} \in \mathbb{Z}_{p}$, and $a=\coprod_{\mathfrak{m}} a_{p}$, where $a_{p} \in$ $\mathbb{Z}[1 / p] / \mathbb{Z}$, then $x \cdot a=\coprod_{\mathfrak{m}} x_{p} a_{p}$. In particular, if $x \in \widehat{\mathbb{Z}}$ and $1 / d \in \mathbb{Q} / \mathbb{Z}$, then $x / d \in \mathbb{Q} / \mathbb{Z}$ is the product of $x(\bmod d \widehat{\mathbb{Z}})$ and $1 / d$. Extend the action of $\mathbb{Z}_{\mathfrak{m}}$ on $\mathbb{Z}[1 / \mathfrak{m}] / \mathbb{Z}$ to all of $\mathbb{Q} / \mathbb{Z}$ via the standard embedding $\mathbb{Z}_{\mathfrak{m}} \rightarrow \widehat{\mathbb{Z}}$, so that the action on the other primary components is trivial.

If $G$ is an abelian group and $d \in \mathbb{N}$, let $G^{d}$ (or $d G$ ) and ${ }_{d} G$ denote the $d$-power (or $d$-multiple for additive notation) and $d$-torsion subgroup of $G$, respectively. Let $|G|$ denote the cardinality of $G$, and let $\exp (G)$, the exponent of $G$, denote the smallest number $m \in \mathbb{N}$ such that $G^{m}=\{1\}$ (or $m G=\{0\}$ ). 
If $G$ is a proabelian group, say a natural number $d$ divides $|G|$ if $d$ divides the order of a finite homomorphic image of $G$. If $\sigma \in G$, say $d$ divides the supernatural number $|\sigma|$ if $d$ divides the order of some finite homomorphic image of $\sigma$.

A subset $\underline{x}=\left\{x_{i}\right\}_{I}$ of a (multiplicative) abelian group $F^{\times}$is $p$-independent for a prime $p$ if for every finite subset $\left\{x_{i_{1}}, \ldots, x_{i_{k}}\right\}, \prod_{j=1}^{k} x_{i_{j}}^{a_{j}} \in F^{\times} p^{r}$ implies $p^{r} \mid a_{j}$, for all $j: 1 \leq j \leq k$. Note $\underline{x}$ is $p$-independent if and only if it is independent $\left(\bmod F^{\times} p\right)$. Say $\underline{x}$ is $\mathfrak{m}$-independent for some $\mathfrak{m} \subset \mathcal{P}$ if it is $p$-independent for all $p \in \mathfrak{m}$. Say $\underline{x}$ is $\mathbb{Z}$-independent if it is $\mathcal{P}$-independent. A subgroup $H$ of an abelian group $F^{\times}$is pure if whenever an element of $H$ has an $n$-th root in $F^{\times}$, it has an $n$-th root in $H$. A pure subset of $F^{\times}$is a subset that generates a pure subgroup.

\section{Bilinear forms, ALternating Forms, AND COHOMOlogy}

By Pontrjagin Duality [RZ, 2.9.6], the category of proabelian groups is dual to the category of discrete torsion abelian groups. In particular, there is an exact contravariant functor "* " from proabelian groups to discrete torsion abelian groups, sending a group $G$ to its group of characters $G^{*}=\operatorname{Hom}(G, \mathbb{Q} / \mathbb{Z})$ (the group of continuous homomorphisms into the discrete group $\mathbb{Q} / \mathbb{Z}$ ), and sending a homomorphism $f$ to its transpose $f^{*}$, defined by the composition $f^{*}\left(\phi^{*}\right)=\phi^{*} \cdot f$, for $\phi^{*} \in G^{*}$. In $[\mathrm{RZ}]$, it is shown that there is a natural isomorphism $G \simeq G^{* *}=\operatorname{Hom}\left(G^{*}, \mathbb{Q} / \mathbb{Z}\right)$.

Suppose $G$ is a proabelian group, and $\mathbb{Q} / \mathbb{Z}$ is the continuous $G$-module with trivial action. Then $G^{*}=\mathrm{H}^{1}(G, \mathbb{Q} / \mathbb{Z})$, and clearly ${ }_{d} G^{*}=\mathrm{H}^{1}\left(G, \frac{1}{d} \mathbb{Z} / \mathbb{Z}\right)$. In this section it will be shown that cup products of characters, which are naturally 2cocycles with coefficients in $\mathbb{Q} / \mathbb{Z}$, are also bilinear forms, which can be used to construct alternating forms on $G$. This construction will be used to prove that the group of alternating forms is naturally isomorphic to the Galois cohomology group $\mathrm{H}^{2}(G, \mathbb{Q} / \mathbb{Z})$, and in particular that $\mathrm{H}^{2}(G, \mathbb{Q} / \mathbb{Z})$ is generated by cup products. This isomorphism reduces the theory of these groups to the theory of $G^{*}$. In particular, the functoriality of the cup product allows the computation of restriction, inflation, and corestriction in terms of corresponding maps on character groups.

Definition 2.1. Suppose $G$ is a proabelian group, and $\mathbb{Q} / \mathbb{Z}$ is the continuous $G$-module with trivial action.

a. Denote by $\operatorname{Bil}(G, \mathbb{Q} / \mathbb{Z})$ the group of continuous bilinear forms on $G$ with values in $\mathbb{Q} / \mathbb{Z}$. If $\boldsymbol{b} \in \operatorname{Bil}(G, \mathbb{Q} / \mathbb{Z})$, set $\operatorname{rad}_{\boldsymbol{b}}(G)=\{\sigma \in G: \boldsymbol{b}(\sigma, G)=\boldsymbol{b}(G, \sigma)=0\}$, the radical of $G$ with respect to $\boldsymbol{b}$. Since $\boldsymbol{b}$ is continuous, $\operatorname{rad}_{\boldsymbol{b}}(G) \leq G$ is an open subgroup.

b. Denote by $\operatorname{Bil}(G, \mathbb{Q} / \mathbb{Z})_{\text {sym }}$ the group of continuous symmetric bilinear forms, the subgroup of $\operatorname{Bil}(G, \mathbb{Q} / \mathbb{Z})$ consisting of $\boldsymbol{b}$ such that $\boldsymbol{b}(\sigma, \tau)=\boldsymbol{b}(\tau, \sigma)$ for all $\sigma, \tau \in G$.

c. Denote by $\operatorname{Alt}(G, \mathbb{Q} / \mathbb{Z})$ the group of continuous alternating bilinear forms, the subgroup of $\operatorname{Bil}(G, \mathbb{Q} / \mathbb{Z})$ consisting of $\boldsymbol{a}$ such that $\boldsymbol{a}(\sigma, \sigma)=0$ for all $\sigma \in G$. Note that $\boldsymbol{a}(\sigma, \tau)=-\boldsymbol{a}(\tau, \sigma)$ and $\operatorname{rad}_{\boldsymbol{a}}(G)=\{\sigma \in G: \boldsymbol{a}(\sigma, G)=0\}$.

If $\boldsymbol{b} \in \operatorname{Bil}(G, \mathbb{Q} / \mathbb{Z})$, let $\tilde{\boldsymbol{b}}$ denote the bilinear form defined by $\tilde{\boldsymbol{b}}(\sigma, \tau)=\boldsymbol{b}(\tau, \sigma)$. Define a map

$$
\text { alt }: \operatorname{Bil}(G, \mathbb{Q} / \mathbb{Z}) \longrightarrow \operatorname{Bil}(G, \mathbb{Q} / \mathbb{Z})
$$

by the rule alt $(\boldsymbol{b})=\boldsymbol{b}-\tilde{\boldsymbol{b}}$. 
Lemma 2.2. Let $\operatorname{Bil}(G, \mathbb{Q} / \mathbb{Z})_{\mathrm{sym}}:=\{\boldsymbol{b} \in \operatorname{Bil}(G, \mathbb{Q} / \mathbb{Z}): \boldsymbol{b}(\sigma, \tau)=\boldsymbol{b}(\tau, \sigma) \forall \sigma, \tau \in$ $G\}$ denote the group of symmetric bilinear forms. The sequence

$$
0 \longrightarrow \operatorname{Bil}(G, \mathbb{Q} / \mathbb{Z})_{\text {sym }} \longrightarrow \operatorname{Bil}(G, \mathbb{Q} / \mathbb{Z}) \stackrel{\text { alt }}{\longrightarrow} \operatorname{Alt}(G, \mathbb{Q} / \mathbb{Z}) \longrightarrow 0
$$

is exact.

Proof. The kernel of alt is obviously $\operatorname{Bil}(G, \mathbb{Q} / \mathbb{Z})_{\text {sym }}$, and the image is clearly contained in $\operatorname{Alt}(G, \mathbb{Q} / \mathbb{Z})$. It remains to show the image is all of $\operatorname{Alt}(G, \mathbb{Q} / \mathbb{Z})$. Suppose $\boldsymbol{a} \in \operatorname{Alt}(G, \mathbb{Q} / \mathbb{Z})$. Since $\boldsymbol{a}$ is continuous, it is defined on a finite quotient of $G$. Order a basis $\left\{\phi_{1}, \ldots, \phi_{n}\right\}$ for this quotient, that is, a set of generators for a cyclic decomposition of the quotient. Define $\boldsymbol{b}$ on the quotient by $\boldsymbol{b}\left(\phi_{i}, \phi_{j}\right)=0$ for each $j \leq i$, and $\boldsymbol{b}\left(\phi_{i}, \phi_{j}\right)=\boldsymbol{a}\left(\phi_{i}, \phi_{j}\right)$ for each $i<j$. Inflate $\boldsymbol{b}$ to all of $G$; then alt $(\boldsymbol{b})=\boldsymbol{a}$.

\section{The Cup Product.}

\section{Setup 2.3.}

Choose $m, n \in \mathbb{N}$, and let $d=\operatorname{gcd}(m, n)$. Let $\nu_{m, n}$ denote the canonical injection

$$
\nu_{m, n}:\left(\frac{1}{m} \mathbb{Z} / \mathbb{Z}\right) \otimes_{\mathbb{Z}}\left(\frac{1}{n} \mathbb{Z} / \mathbb{Z}\right) \longrightarrow \mathbb{Q} / \mathbb{Z}
$$

defined by $\nu_{m, n}\left(\frac{a}{m} \otimes \frac{b}{n}\right)=\frac{a b}{d}$. The image is $\frac{1}{d} \mathbb{Z} / \mathbb{Z}$. Use the notation $\nu_{d}$ if $m=n=d$. It is easily verified that if $m^{\prime}\left|m, n^{\prime}\right| n$, and $d^{\prime}=\operatorname{gcd}\left(m^{\prime}, n^{\prime}\right)$, then there is a commutative diagram

$$
\left(d / d^{\prime}\right) \cdot \nu_{m, n}=\nu_{m^{\prime}, n^{\prime}} \cdot\left(\frac{m}{m^{\prime}} \otimes \frac{n}{n^{\prime}}\right),
$$

where $\frac{m}{m^{\prime}} \otimes \frac{n}{n^{\prime}} \in \mathbb{Z} \otimes \mathbb{Z}$ is the multiplication map. In particular, $\nu_{m, n}=\nu_{d} \cdot\left(\frac{m}{d} \otimes \frac{n}{d}\right)$. On the other hand, the natural map $\iota^{\otimes 2}: \frac{1}{m^{\prime}} \mathbb{Z} / \mathbb{Z} \otimes \frac{1}{n^{\prime}} \mathbb{Z} / \mathbb{Z} \rightarrow \frac{1}{m} \mathbb{Z} / \mathbb{Z} \otimes \frac{1}{n} \mathbb{Z} / \mathbb{Z}$ produces the diagram

$$
\left(\operatorname{lcm}[m, n] / \operatorname{lcm}\left[m^{\prime}, n^{\prime}\right]\right) \cdot \nu_{m^{\prime}, n^{\prime}}=\nu_{m, n} \cdot \iota^{\otimes 2} .
$$

In particular, $(\operatorname{lcm}[m, n] / d) \cdot \nu_{d}=\nu_{m, n} \cdot \iota^{\otimes 2}$.

It will be shown below that all bilinear forms arise from the cup product, as follows. Since $G$ acts trivially on $\mathbb{Q} / \mathbb{Z}$, the cup product pairing is defined by

$$
\begin{aligned}
{ }_{m} G^{*} \times{ }_{n} G^{*} & \longrightarrow \mathrm{H}^{2}(G, \mathbb{Q} / \mathbb{Z}) \\
\left(\phi^{*}, \psi^{*}\right) & \longmapsto\left[\phi^{*} \otimes_{(m, n)} \psi^{*}\right]
\end{aligned}
$$

where $\phi^{*} \otimes_{(m, n)} \psi^{*}(\sigma, \tau)=\nu_{m, n}\left(\phi^{*}(\sigma) \otimes \psi^{*}(\tau)\right)$. Use the shorthand notation $\phi^{*} \otimes_{d}$ $\psi^{*}$ if $m=n=d$. Since the tensor product is bilinear, this pairing factors through $\operatorname{Bil}(G, \mathbb{Q} / \mathbb{Z})$,

$$
\begin{aligned}
{ }_{m} G^{*} \times{ }_{n} G^{*} & \longrightarrow \operatorname{Bil}(G, \mathbb{Q} / \mathbb{Z}) \\
\left(\phi^{*}, \psi^{*}\right) & \longmapsto \phi^{*} \otimes_{(m, n)} \psi^{*}
\end{aligned}
$$

The cup product is functorial in $G$, so that if $f: H \rightarrow G$ is a homomorphism with transpose $f^{*}: G^{*} \rightarrow H^{*}$, then $f^{*}\left({ }_{m} G^{*}\right) \leq{ }_{m} H^{*}, f^{*}\left({ }_{n} G^{*}\right) \leq{ }_{n} H^{*}$, and

$$
f^{* \otimes 2}\left(\phi^{*} \otimes_{(m, n)} \psi^{*}\right)=f^{*}\left(\phi^{*}\right) \otimes_{(m, n)} f^{*}\left(\psi^{*}\right) .
$$

The order of an element $\phi^{*} \otimes_{(m, n)} \psi^{*} \in \operatorname{Bil}(G, \mathbb{Q} / \mathbb{Z})$ obviously depends on $m$ and $n$. For example, $\left(\frac{m}{d} \phi^{*}\right) \otimes_{d}\left(\frac{n}{d} \psi^{*}\right)$ has order $d$, while by $(2.3 .2),\left(\frac{m}{d} \phi^{*}\right) \otimes_{(m, n)}\left(\frac{n}{d} \psi^{*}\right)$ has order $d^{2} / \operatorname{gcd}\left(d^{2}, \operatorname{lcm}[m, n]\right)$.

Let ${ }_{m} G^{*} \otimes{ }_{n} G^{*}$ denote the image in $\operatorname{Bil}(G, \mathbb{Q} / \mathbb{Z})$ of the cup product pairing on ${ }_{m} G^{*} \times{ }_{n} G^{*}$. By (2.3.1), every element of ${ }_{m} G^{*} \otimes_{n} G^{*}$ is also in the image of ${ }_{d} G^{*} \otimes_{d} G^{*}$. 


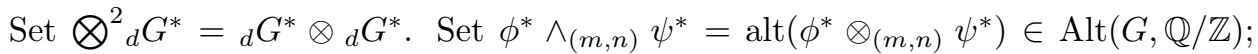
then $\phi^{*} \wedge_{(m, n)} \psi^{*}=\phi^{*} \otimes_{(m, n)} \psi^{*}-\psi^{*} \otimes_{(m, n)} \phi^{*}$. Let ${ }_{m} G^{*} \wedge_{n} G^{*}$ denote the image in $\operatorname{Alt}(G, \mathbb{Q} / \mathbb{Z})$ of $\operatorname{alt}\left({ }_{m} G^{*} \otimes{ }_{n} G^{*}\right)$, and set $\bigwedge^{2}{ }_{d} G^{*}=\operatorname{alt}\left(\otimes^{2}{ }_{d} G^{*}\right)$. Finally put

$$
\begin{aligned}
\bigotimes^{2} G^{*} & =\bigcup_{d \in \mathbb{N}}\left(\bigotimes^{2}{ }_{d} G^{*}\right) \leq \operatorname{Bil}(G, \mathbb{Q} / \mathbb{Z}), \\
\bigwedge^{2} G^{*} & =\bigcup_{d \in \mathbb{N}}\left(\bigwedge^{2}{ }_{d} G^{*}\right) \leq \operatorname{Alt}(G, \mathbb{Q} / \mathbb{Z}) .
\end{aligned}
$$

Theorem 2.4. Suppose $G$ is a proabelian group. Then $\bigotimes^{2} G^{*}=\operatorname{Bil}(G, \mathbb{Q} / \mathbb{Z})$ and $\bigwedge^{2} G^{*}=\operatorname{Alt}(G, \mathbb{Q} / \mathbb{Z})$. If $G$ is torsion-free, then ${ }_{d} \operatorname{Bil}(G, \mathbb{Q} / \mathbb{Z})=\bigotimes^{2}{ }_{d} G^{*}$ and ${ }_{d} \operatorname{Alt}(G, \mathbb{Q} / \mathbb{Z})=\bigwedge^{2}{ }_{d} G^{*}$. The natural map

$$
\operatorname{Bil}(G, \mathbb{Q} / \mathbb{Z}) \longrightarrow \mathrm{H}^{2}(G, \mathbb{Q} / \mathbb{Z})
$$

defined by the cup product $\phi^{*} \otimes_{d} \psi^{*} \mapsto\left[\phi^{*} \otimes_{d} \psi^{*}\right]$ is surjective, with kernel $\operatorname{Bil}(G, \mathbb{Q} / \mathbb{Z})_{\text {sym. }}$. In particular, $\mathrm{H}^{2}(G, \mathbb{Q} / \mathbb{Z})$ is generated by cup products. The induced homomorphism

$$
\text { alt : } \mathrm{H}^{2}(G, \mathbb{Q} / \mathbb{Z}) \stackrel{\sim}{\longrightarrow} \operatorname{Alt}(G, \mathbb{Q} / \mathbb{Z}),
$$

defined by $\left[\phi^{*} \otimes_{d} \psi^{*}\right] \mapsto \phi^{*} \wedge_{d} \psi^{*}$, is an isomorphism, functorial in $G$.

Proof. The functoriality of all of these maps is an immediate consequence of the functoriality of the cup product. First show $\bigotimes^{2} G^{*}=\operatorname{Bil}(G, \mathbb{Q} / \mathbb{Z})$. Suppose $\boldsymbol{b} \in$ $\operatorname{Bil}(G, \mathbb{Q} / \mathbb{Z})$ has order $d$. By inflation it is enough to prove the result for $G$ finite. Then $G$ has a basis $\left\{\phi_{i}: 1 \leq i \leq n\right\}$, and $\boldsymbol{b}$ is completely determined by its values on the basis $\left\{\left(\phi_{i}, \phi_{j}\right): 1 \leq i, j \leq n\right\}$ for $G \times G$. Let $\left\{\phi_{i}^{*}\right\}$ be the basis for ${ }_{d} G^{*}$ dual to $\left\{\phi_{i}\right\}$, defined by $\phi_{i}^{*}\left(\phi_{j}\right)=\delta_{i j} / \operatorname{gcd}\left(d,\left|\phi_{i}\right|\right)$, where $\delta_{i j}$ is the Kronecker delta function. Let $d_{i}=\operatorname{gcd}\left(d,\left|\phi_{i}\right|\right)=\left|\phi_{i}^{*}\right|$, and set $d_{i j}=\operatorname{gcd}\left(d_{i}, d_{j}\right)$. Then $\phi_{i}^{*} \otimes_{\left(d_{i}, d_{j}\right)} \phi_{j}^{*}$ has order $d_{i j}$, and since $\boldsymbol{b}$ is bilinear, $\boldsymbol{b}\left(\phi_{i}, \phi_{j}\right)=a_{i j} / d_{i j}$ for some $a_{i j}$. By definition, $\left(\phi_{i}^{*} \otimes_{\left(d_{i}, d_{j}\right)} \phi_{j}^{*}\right)\left(\phi_{k}, \phi_{l}\right)=\delta_{i k} \delta_{j l} / d_{i j}$. Thus $\boldsymbol{b}=\sum_{i, j} a_{i j}\left(\phi_{i}^{*} \otimes_{\left(d_{i}, d_{j}\right)} \phi_{j}^{*}\right)$. Since by (2.3.1) each $\phi_{i}^{*} \otimes_{\left(d_{i}, d_{j}\right)} \phi_{j}^{*}$ is represented by the element $\left(\frac{d_{i}}{d_{i j}} \phi_{i}^{*}\right) \otimes_{d_{i j}}\left(\frac{d_{j}}{d_{i j}} \phi_{j}^{*}\right)$ of $\bigotimes^{2}{ }_{d_{i j}} G^{*}$, this proves $\bigotimes^{2} G^{*}=\operatorname{Bil}(G, \mathbb{Q} / \mathbb{Z})$. By $(2.3 .2)$,

$$
\left(d / \operatorname{lcm}\left[d_{i}, d_{j}\right]\right)\left(\phi_{i}^{*} \otimes_{\left(d_{i}, d_{j}\right)} \phi_{j}^{*}\right)=\phi_{i}^{*} \otimes_{d} \phi_{j}^{*}
$$

If $G$ is torsion-free, then by Pontrjagin duality $G^{*}$ is divisible, hence $d / \operatorname{lcm}\left[d_{i}, d_{j}\right]$ is relatively prime to $d_{i j}$. Therefore $\phi_{i}^{*} \otimes_{\left(d_{i}, d_{j}\right)} \phi_{j}^{*} \in \otimes^{2}{ }_{d} G^{*}$ for all $i$ and $j$. Therefore

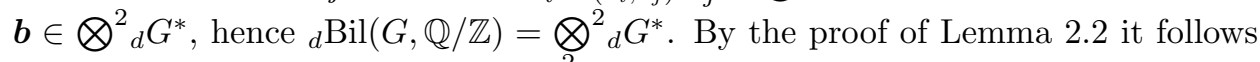
immediately that ${ }_{d} \operatorname{Alt}(G, \mathbb{Q} / \mathbb{Z})=\bigwedge^{2}{ }_{d} G^{*}$.

Extend alt to $\mathrm{Z}^{2}(G, \mathbb{Q} / \mathbb{Z})$ by setting alt $(f)=f-\tilde{f}$ for all $f \in \mathrm{Z}^{2}(G, \mathbb{Q} / \mathbb{Z})$, where $\tilde{f}$ is defined by $\tilde{f}(\sigma, \tau)=f(\tau, \sigma)$, for all $\sigma, \tau \in G$. It is straightforward to verify that, because $G$ is abelian and the action of $G$ on $\mathbb{Q} / \mathbb{Z}$ is trivial, $\partial \tilde{f}(\rho, \sigma, \tau)=$ $-\partial f(\tau, \sigma, \rho)=0$, hence $\tilde{f}$ is a cocycle. Moreover, $f-\tilde{f}$ is indeed an alternating form, for it is clear from the definition that $(f-\tilde{f})(\sigma, \tau)=-(f-\tilde{f})(\tau, \sigma)$, and the alternating sum of the cocycle condition on $f$ applied successively to the triples $(\rho, \sigma, \tau),(\rho, \tau, \sigma)$, and $(\tau, \rho, \sigma)$ shows that $f-\tilde{f}$ is linear on the left, hence bilinear [AT, Section 1].

The kernel of alt $: \mathrm{Z}^{2}(G, \mathbb{Q} / \mathbb{Z}) \rightarrow \operatorname{Alt}(G, \mathbb{Q} / \mathbb{Z})$ is $\mathrm{Z}^{2}(G, \mathbb{Q} / \mathbb{Z})_{\text {sym }}=\{f: f=\tilde{f}\}$. Since the action of $G$ on $\mathbb{Q} / \mathbb{Z}$ is trivial, a coboundary $f \in \mathrm{B}^{2}(G, \mathbb{Q} / \mathbb{Z})$ is defined by 
$\partial h(\sigma, \tau)=h(\tau)-h(\sigma \tau)+h(\sigma)$ for some $h: G \rightarrow \mathbb{Q} / \mathbb{Z}$. Therefore since $G$ is abelian, $\mathrm{Z}^{2}(G, \mathbb{Q} / \mathbb{Z})_{\text {sym }}$ contains $\mathrm{B}^{2}(G, \mathbb{Q} / \mathbb{Z})$, and alt : $\mathrm{H}^{2}(G, \mathbb{Q} / \mathbb{Z}) \rightarrow \operatorname{Alt}(G, \mathbb{Q} / \mathbb{Z})$ is well defined. Let $\mathrm{H}^{2}(G, \mathbb{Q} / \mathbb{Z})_{\text {sym }}$ denote the kernel, equal to $\mathrm{Z}^{2}(G, \mathbb{Q} / \mathbb{Z})_{\text {sym }} / \mathrm{B}^{2}(G, \mathbb{Q} / \mathbb{Z})$.

Claim: $\mathrm{H}^{2}(G, \mathbb{Q} / \mathbb{Z})_{\text {sym }}=0$. For $[f] \in \mathrm{H}^{2}(G, \mathbb{Q} / \mathbb{Z})_{\text {sym }}$ if and only if $f=\tilde{f}$, if and only if $[f]$ defines an abelian group extension of $G$ by $\mathbb{Q} / \mathbb{Z}$. Thus $\mathrm{H}^{2}(G, \mathbb{Q} / \mathbb{Z})_{\text {sym }}=$ $\operatorname{Ext}_{\mathbb{Z}}^{1}(G, \mathbb{Q} / \mathbb{Z})$. Since $\mathbb{Q} / \mathbb{Z}$ is divisible, the claim follows. Now there is a commutative diagram

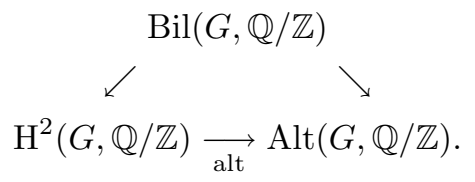

Since alt $: \operatorname{Bil}(G, \mathbb{Q} / \mathbb{Z}) \rightarrow \operatorname{Alt}(G, \mathbb{Q} / \mathbb{Z})$ is surjective by Lemma 2.2 , alt $: \mathrm{H}^{2}(G, \mathbb{Q} / \mathbb{Z})$ $\rightarrow \operatorname{Alt}(G, \mathbb{Q} / \mathbb{Z})$, is surjective, hence bijective. It follows that the natural map $\operatorname{Bil}(G, \mathbb{Q} / \mathbb{Z}) \rightarrow \mathrm{H}^{2}(G, \mathbb{Q} / \mathbb{Z})$ is surjective, with kernel $\operatorname{Bil}(G, \mathbb{Q} / \mathbb{Z})_{\text {sym. }}$. This completes the proof.

Remark 2.5. A similar result appears in [AT. If $G$ is any group and $A$ is a trivial $G$-module, then by the Universal Coefficient Theorem, the sequence

$$
0 \longrightarrow \operatorname{Ext}_{\mathbb{Z}}^{1}\left(\mathrm{H}_{1}(G, \mathbb{Z}), A\right) \longrightarrow \mathrm{H}^{2}(G, A) \longrightarrow \operatorname{Hom}\left(\mathrm{H}_{2}(G, \mathbb{Z}), A\right) \longrightarrow 0
$$

is (split) exact. If $G$ is abelian, $\mathrm{H}_{1}(G, \mathbb{Z})=G, \mathrm{H}_{2}(G, \mathbb{Z}) \simeq \bigwedge^{2} G$, and the projection is given by $[f] \mapsto f-\tilde{f}$. This is [AT, Proposition 1.3]. If $A$ is divisible, this is Theorem 2.4. More generally, if $A$ is divisible and $G$ is any group, then $\mathrm{H}^{2}(G, A) \stackrel{\sim}{\longrightarrow}$ $\operatorname{Hom}\left(\mathrm{H}_{2}(G, \mathbb{Z}), A\right)$.

\section{Functoriality.}

Setup 2.6. For the next few results, suppose

$$
1 \longrightarrow H \stackrel{\iota}{\longrightarrow} G \stackrel{\pi}{\longrightarrow} \bar{G} \longrightarrow 1
$$

is an exact sequence of proabelian groups, such that $\bar{G}$ has finite exponent $r$. Since "*" is an exact functor, there is a dual exact sequence

$$
0 \longrightarrow \bar{G}^{*} \stackrel{\pi^{*}}{\longrightarrow} G^{*} \stackrel{\iota^{*}}{\longrightarrow} H^{*} \longrightarrow 0 .
$$

The dual $\iota^{*}: G^{*} \rightarrow H^{*}$ is the restriction map, and $\pi^{*}: \bar{G}^{*} \rightarrow G^{*}$ is the inflation. If $G$ is torsion-free, then $G^{*}$ and $H^{*}$ are divisible.

For each integer $d$ divisible by $r$, let $\jmath_{d}$ denote the composite of exponentiation by $d$ and $\iota^{-1}$,

$$
\jmath_{d}: G \rightarrow G^{d} \hookrightarrow H .
$$

Its dual is $\jmath_{d}^{*}: H^{*} \rightarrow\left(G^{d}\right)^{*} \hookrightarrow G^{*}$. If $\iota$ is open and $s=|\bar{G}|<\infty$, then by S. VII.8.Proposition 7], $\jmath:=\jmath_{s}$ is the classical transfer homomorphism, and its dual $\jmath^{*}: H^{*} \rightarrow G^{*}$ is the cohomological corestriction. Since $\iota^{*}$ is restriction, the composition $\jmath_{d}^{*} \cdot \iota^{*}: G^{*} \rightarrow G^{*}$ is multiplication by $d$. On the other hand, since $\iota^{*}$ is surjective, $\iota^{*} \cdot \jmath_{d}^{*}\left(H^{*}\right)=\iota^{*} \cdot \jmath_{d}^{*} \cdot \iota^{*}\left(G^{*}\right)=\iota^{*}\left(d G^{*}\right)=d \iota^{*}\left(G^{*}\right)=d H^{*}$. Thus $\iota^{*} \cdot \jmath_{d}^{*}: H^{*} \rightarrow H^{*}$ is also multiplication by $d$. 
Lemma 2.7. Assume Setup 2.6. For all $d \in \mathbb{N}$ divisible by $r$, there is a natural exact sequence,

$$
0 \longrightarrow{ }_{d} \bar{G}^{*} \stackrel{\pi^{*}}{\longrightarrow}{ }_{d} G^{*} \stackrel{\iota^{*}}{\longrightarrow}{ }_{d} H^{*} \stackrel{\jmath_{d}^{*}}{\longrightarrow} \pi^{*}\left(\bar{G}^{*}\right) .
$$

If $G$ is torsion-free, the right arrow $\jmath_{d}^{*}$ is surjective. In particular, if $G$ is torsion-free and $\iota$ is open, $\jmath^{*}\left({ }_{s} H^{*}\right) \simeq \bar{G}^{*}$, where $s=|\bar{G}|$ and $\jmath$ is the transfer.

Proof. Consider the commutative diagram

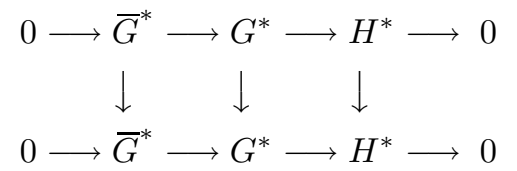

where the vertical arrows are multiplication by $d$. By the Snake Lemma, the sequence

$$
0 \longrightarrow{ }_{d} \bar{G}^{*} \longrightarrow{ }_{d} G^{*} \longrightarrow{ }_{d} H^{*} \stackrel{\jmath_{d}^{*}}{\longrightarrow} \pi^{*}\left(\bar{G}^{*}\right) / \pi^{*}\left(d \bar{G}^{*}\right) \longrightarrow G^{*} / d G^{*} \longrightarrow 0
$$

is exact. Since $r$ divides $d, d \bar{G}^{*}=\{0\}$, so $\pi^{*}\left(\bar{G}^{*}\right) / \pi^{*}\left(d \bar{G}^{*}\right)=\pi^{*}\left(\bar{G}^{*}\right)$, as desired. If $G$ is torsion-free, then $G^{*}$ is divisible, and then $G^{*} / d G^{*}=\{0\}$, i.e., $\jmath_{d}^{*}$ is surjective. This completes the proof.

Assume Setup 2.6. By [N, Proposition 1.5.3], $\iota$ and $\pi$ induce the cohomological restriction and inflation maps on $\mathrm{H}^{2}(G, \mathbb{Q} / \mathbb{Z})$ and $\mathrm{H}^{2}(\bar{G}, \mathbb{Q} / \mathbb{Z})$, i.e., res $=\left[\iota^{* \otimes 2}\right]$ and inf $=\left[\pi^{* \otimes 2}\right]$. By the definition of alt, $\iota^{* \wedge 2}$ and $\pi^{* \wedge 2}$ are the restriction and inflation maps on $\operatorname{Alt}(G, \mathbb{Q} / \mathbb{Z})$ and $\operatorname{Alt}(\bar{G}, \mathbb{Q} / \mathbb{Z})$, so that the maps

$$
\begin{aligned}
& \text { res : } \operatorname{Alt}(G, \mathbb{Q} / \mathbb{Z}) \longrightarrow \operatorname{Alt}(H, \mathbb{Q} / \mathbb{Z}), \quad \inf : \operatorname{Alt}(\bar{G}, \mathbb{Q} / \mathbb{Z}) \longrightarrow \operatorname{Alt}(G, \mathbb{Q} / \mathbb{Z}) \text {, } \\
& \boldsymbol{a} \longmapsto \iota^{* \wedge 2}(\boldsymbol{a}) \quad \boldsymbol{b} \longmapsto \pi^{* \wedge 2}(\boldsymbol{b})
\end{aligned}
$$

are given explicitly by $\operatorname{res}(\boldsymbol{a})(\sigma, \tau)=\boldsymbol{a}(\iota(\sigma), \iota(\tau))$ for each $\boldsymbol{a} \in \operatorname{Alt}(G, \mathbb{Q} / \mathbb{Z})$, where $\sigma, \tau \in H$, and $\inf (\overline{\boldsymbol{a}})(\sigma, \tau)=\overline{\boldsymbol{a}}(\pi(\sigma), \pi(\tau))$ for each $\overline{\boldsymbol{a}} \in \operatorname{Alt}(\bar{G}, \mathbb{Q} / \mathbb{Z})$, where $\sigma, \tau \in$ $G$. On elementary elements $\phi^{*} \wedge_{d} \psi^{*} \in \operatorname{Alt}(G, \mathbb{Q} / \mathbb{Z})$ and $\bar{\phi}^{*} \wedge_{d} \bar{\psi}^{*} \in \operatorname{Alt}(\bar{G}, \mathbb{Q} / \mathbb{Z})$, compute res $\left(\phi^{*} \wedge_{d} \psi^{*}\right)=\iota^{*}\left(\phi^{*}\right) \wedge_{d} \iota^{*}\left(\psi^{*}\right)$ and $\inf \left(\bar{\phi}^{*} \wedge_{d} \bar{\psi}^{*}\right)=\pi^{*}\left(\bar{\phi}^{*}\right) \wedge_{d} \pi^{*}\left(\bar{\psi}^{*}\right)$.

When $\iota: H \hookrightarrow G$ is open, define the corestriction

$$
\text { cor : } \operatorname{Alt}(H, \mathbb{Q} / \mathbb{Z}) \rightarrow \operatorname{Alt}(G, \mathbb{Q} / \mathbb{Z})
$$

to be alt $($ cor $)$, where cor : $\mathrm{H}^{2}(H, \mathbb{Q} / \mathbb{Z}) \rightarrow \mathrm{H}^{2}(G, \mathbb{Q} / \mathbb{Z})$ is the usual cohomological corestriction. This map, which is induced from the transfer, will be computed in Theorem 2.10.

Theorem 2.8 "Inflation and Restriction". Assume Setup 2.6, with G a general proabelian group. The inflation is injective, and res $\cdot \inf =0$ on $\operatorname{Bil}(\bar{G}, \mathbb{Q} / \mathbb{Z})$, $\mathrm{H}^{2}(\bar{G}, \mathbb{Q} / \mathbb{Z})$, and $\operatorname{Alt}(\bar{G}, \mathbb{Q} / \mathbb{Z})$. If $G$ is torsion-free the restriction surjects onto $\operatorname{Bil}(H, \mathbb{Q} / \mathbb{Z}), \mathrm{H}^{2}(H, \mathbb{Q} / \mathbb{Z})$, and $\operatorname{Alt}(H, \mathbb{Q} / \mathbb{Z})$. The kernel of the restriction in $\mathrm{H}^{2}(G, \mathbb{Q} / \mathbb{Z})$ and $\operatorname{Alt}(G, \mathbb{Q} / \mathbb{Z})$ has exponent dividing the maximum order of all subgroups of $\bar{G}$ of rank at most two, so divides $|\bar{G}|$.

Proof. Inflation is obviously injective on bilinear forms, hence also on alternating forms, since they form a subgroup, and on $\mathrm{H}^{2}(G, \mathbb{Q} / \mathbb{Z})$ by Theorem 2.4. To see that res $\cdot \inf =0$ on $\operatorname{Bil}(\bar{G}, \mathbb{Q} / \mathbb{Z})$, suppose $\boldsymbol{b}=\inf (\boldsymbol{a})$, where $\boldsymbol{a} \in \operatorname{Bil}(\bar{G}, \mathbb{Q} / \mathbb{Z})$, and compute

$$
\operatorname{res}(\boldsymbol{b})(\sigma, \tau)=\boldsymbol{b}(\iota(\sigma), \iota(\tau))=\boldsymbol{a}(\pi \cdot \iota(\sigma), \pi \cdot \iota(\tau))=\boldsymbol{a}(\overline{1}, \overline{1})=0
$$


for all $\sigma, \tau \in H$, as desired. Since they form a subgroup, res $\cdot \inf =0$ on alternating forms as well, and by Theorem 2.4 the result holds on $\mathrm{H}^{2}(G, \mathbb{Q} / \mathbb{Z})$.

Suppose $G$ is torsion-free; then so is $H$, as $H \hookrightarrow G$. Then $G^{*}$ and $H^{*}$ are divisible, and it is easy to see using the cup product that $\operatorname{Bil}(G, \mathbb{Q} / \mathbb{Z})$ and $\operatorname{Bil}(H, \mathbb{Q} / \mathbb{Z})$ are divisible, hence that $\operatorname{res}(\operatorname{Bil}(G, \mathbb{Q} / \mathbb{Z}))$ is divisible or trivial. Suppose $\boldsymbol{b} \in \operatorname{Bil}(H, \mathbb{Q} / \mathbb{Z})$. For any $d$ divisible by $r=\exp (\bar{G}),\left(\iota^{* \otimes 2} \cdot \jmath_{d}^{* \otimes 2}\right)(\boldsymbol{b})=\left(\jmath_{d} \cdot \iota\right)^{* \otimes 2}(\boldsymbol{b})=d^{2} \boldsymbol{b} \in$ $\operatorname{Bil}(H, \mathbb{Q} / \mathbb{Z})$ is in the image of the restriction $\iota^{* \otimes 2}=$ res. Thus the quotient $\operatorname{Bil}(H, \mathbb{Q} / \mathbb{Z}) / \operatorname{res}(\operatorname{Bil}(G, \mathbb{Q} / \mathbb{Z}))$ has bounded exponent, hence is trivial by the divisibility of each group. Thus restriction is surjective. The same argument works on $\operatorname{Alt}(G, \mathbb{Q} / \mathbb{Z})$, and by Theorem 2.4 this proves it also for $\mathrm{H}^{2}(G, \mathbb{Q} / \mathbb{Z})$.

Suppose $\boldsymbol{a} \in \operatorname{ker}(\mathrm{res}) \leq \operatorname{Alt}(G, \mathbb{Q} / \mathbb{Z})$, and $\sigma, \tau \in G$. Let $k$ be the order of $\pi(\sigma)$, and let $l$ the order of $\pi(\tau)$ modulo $\langle\pi(\sigma)\rangle \leq \bar{G}$. Then $k l$ is the order of the subgroup $\langle\pi(\sigma), \pi(\tau)\rangle \leq \bar{G}, \sigma^{k} \in H$, and $\tau^{l}=\sigma^{m} \rho$ for some number $m$ and element $\rho \in \iota(H)$. Now compute using the bilinearity of $\boldsymbol{a}, k \boldsymbol{l} \boldsymbol{a}(\sigma, \tau)=\boldsymbol{a}\left(\sigma^{k}, \sigma^{m}\right)+\boldsymbol{a}\left(\sigma^{k}, \rho\right)$. Since $\boldsymbol{a}$ is alternating, $\boldsymbol{a}\left(\sigma^{k}, \sigma^{m}\right)=0$, and since $\operatorname{res}(\boldsymbol{a})=0, \boldsymbol{a}\left(\sigma^{k}, \rho\right)=0$. Therefore $k l \boldsymbol{a}(\sigma, \tau)=0$. If $n$ is the maximum order of all subgroups of $\bar{G}$ of rank at most two, then $k l \mid n$, and therefore $n \boldsymbol{a}(\sigma, \tau)=0$ for all $\sigma, \tau \in G$, hence $n \boldsymbol{a}=0$. By Theorem 2.4 , the same result holds on $\mathrm{H}^{2}(G, \mathbb{Q} / \mathbb{Z})$. This completes the proof.

Remark 2.9. The last statement of Theorem 2.8 does not hold for $\operatorname{Bil}(G, \mathbb{Q} / \mathbb{Z})$. For suppose $G=\widehat{\mathbb{Z}} \times \widehat{\mathbb{Z}}$ and $H=2 \widehat{\mathbb{Z}} \times \widehat{\mathbb{Z}}$. By Theorem 2.8, the kernel of the restriction on $\mathrm{H}^{2}(G, \mathbb{Q} / \mathbb{Z})$ and $\operatorname{Alt}(G, \mathbb{Q} / \mathbb{Z})$ has exponent dividing 2 , since $|\bar{G}|=2$. But if $\boldsymbol{b} \in \operatorname{Bil}(G, \mathbb{Q} / \mathbb{Z})$ is defined on the standard basis $\left\{\phi_{1}, \phi_{2}\right\}$ of $G$ by $\boldsymbol{b}\left(\phi_{i}, \phi_{j}\right)=$ $\delta_{i 1} \delta_{j 1} / 4$, then $\operatorname{res}(\boldsymbol{b})=0$ and $|\boldsymbol{b}|=r^{2}=4$. In general, the kernel of the restriction on $\operatorname{Bil}(G, \mathbb{Q} / \mathbb{Z})$ has exponent dividing $r^{2}=\exp (\bar{G})^{2}$, since if $\operatorname{res}(\boldsymbol{b})=0$, then $r^{2} \boldsymbol{b}(\sigma, \tau)=\boldsymbol{b}\left(\sigma^{r}, \tau^{r}\right)=0$ for all $\sigma, \tau \in G$.

Theorem 2.10 "Corestriction". Assume Setup 2.6, with $\iota: H \rightarrow G$ an open embedding of torsion-free proabelian groups, $s=|\bar{G}|$, and $\jmath: G \rightarrow H$ the transfer. For each $\boldsymbol{a}$ in $\operatorname{Alt}(H, \mathbb{Q} / \mathbb{Z})$ and $\boldsymbol{b} \in \operatorname{Bil}(H, \mathbb{Q} / \mathbb{Z})$, let $\frac{1}{s} \boldsymbol{a}$ and $\frac{1}{s} \boldsymbol{b}$ be any elements such that $s \cdot \frac{1}{s} \boldsymbol{a}=\boldsymbol{a}$ and $s \cdot \frac{1}{s} \boldsymbol{b}=\boldsymbol{b}$. Then

$$
\begin{array}{rlrl}
\operatorname{cor}: \operatorname{Alt}(H, \mathbb{Q} / \mathbb{Z}) & \longrightarrow \operatorname{Alt}(G, \mathbb{Q} / \mathbb{Z}), \quad \operatorname{cor}: \mathrm{H}^{2}(H, \mathbb{Q} / \mathbb{Z}) & \longrightarrow \mathrm{H}^{2}(G, \mathbb{Q} / \mathbb{Z}), \\
\boldsymbol{a} & \longmapsto J^{* \wedge 2}\left(\frac{1}{s} \boldsymbol{a}\right) & {[\boldsymbol{b}]} & \longmapsto\left[\jmath^{* \otimes 2}\right]\left(\left[\frac{1}{s} \boldsymbol{b}\right]\right) .
\end{array}
$$

The corestriction on elementary elements $\phi^{*} \wedge_{d} \psi^{*} \in \operatorname{Alt}(G, \mathbb{Q} / \mathbb{Z})$ and $\left[\phi^{*} \otimes_{d} \psi^{*}\right] \in$ $\mathrm{H}^{2}(G, \mathbb{Q} / \mathbb{Z})$, where $\phi^{*}, \psi^{*} \in{ }_{d} H^{*}$, is computed as follows. Let $\frac{1}{s} \phi^{*}, \frac{1}{s} \psi^{*} \in{ }_{s d} H^{*}$ be $s$-th roots. Then

$\left.\operatorname{cor}\left(\phi^{*} \wedge_{d} \psi^{*}\right)=\jmath^{*}\left(\frac{1}{s} \phi^{*}\right) \wedge_{s d} \jmath^{*}\left(\frac{1}{s} \psi^{*}\right)\right), \quad \operatorname{cor}\left(\left[\phi^{*} \otimes_{d} \psi^{*}\right]\right)=\left[\jmath^{*}\left(\frac{1}{s} \phi^{*}\right) \otimes_{s d} \jmath^{*}\left(\frac{1}{s} \psi^{*}\right)\right]$.

Proof. By Theorem 2.4, it suffices to prove the theorem for alternating forms. Since $H$ is torsion-free, $H^{*}$ is divisible, hence $\operatorname{Alt}(H, \mathbb{Q} / \mathbb{Z})$ is divisible, so $\frac{1}{s} \boldsymbol{a}$ exists. To show the given map is well defined, suppose $\boldsymbol{c} \in \operatorname{Alt}(H, \mathbb{Q} / \mathbb{Z})$ has order dividing $s$. Since $G$ is torsion-free, by Theorem 2.8 there exists an element $\boldsymbol{b} \in \operatorname{Alt}(G, \mathbb{Q} / \mathbb{Z})$ such that $\operatorname{res}(\boldsymbol{b})=\boldsymbol{c}$, and $|\boldsymbol{b}|$ divides $|\boldsymbol{c}| s$. In particular, $s^{2} \boldsymbol{b}=0$. By the functoriality of the cup product, $\bigwedge^{2}{ }_{d} H^{*}={ }_{d} \operatorname{Alt}(H, \mathbb{Q} / \mathbb{Z})$ is covariant on ${ }_{d} H^{*}$, and it follows that $\jmath^{* \wedge 2} \iota^{* \wedge 2}=\left(\jmath^{*} \iota^{*}\right)^{\wedge 2}$. Since $\jmath^{*} \iota^{*}$ is multiplication by $s, \jmath^{* \wedge 2} \iota^{* \wedge 2}$ is multiplication by $s^{2}$. Therefore $\jmath^{* \wedge 2}(\boldsymbol{c})=\jmath^{* \wedge 2} \iota^{* \wedge 2}(\boldsymbol{b})=0$, and the given map is well defined. Since $\jmath^{* \wedge 2}$ is a homomorphism and $s\left(\frac{1}{s}\left(\boldsymbol{a}+\boldsymbol{a}^{\prime}\right)\right)=\boldsymbol{a}+\boldsymbol{a}^{\prime}=s \frac{1}{s} \boldsymbol{a}+s \frac{1}{s} \boldsymbol{a}^{\prime}$, the map $\boldsymbol{a} \mapsto \jmath^{* \wedge 2}\left(\frac{1}{s} \boldsymbol{a}\right)$ is a homomorphism. 
Now to show the given map is the corestriction. Since res $=\iota^{* \wedge 2}$ is a surjective homomorphism, and cor $\cdot$ res is multiplication by $s$, it suffices to prove that if $\boldsymbol{b} \in$ $\operatorname{Alt}(G, \mathbb{Q} / \mathbb{Z})$, then $\jmath^{* \wedge 2}\left(\frac{1}{s} \iota^{* \wedge 2}(\boldsymbol{b})\right)=s \boldsymbol{b}$. Since $\iota^{* \wedge 2}$ is a homomorphism, $\frac{1}{s} \iota^{* \wedge 2}(\boldsymbol{b})$ may be replaced by $\iota^{* \wedge 2}\left(\frac{1}{s} \boldsymbol{b}\right)$, where $\frac{1}{s} \boldsymbol{b}$ is any $s$-th root of $\boldsymbol{b}$. It has already been seen that $\jmath^{* \wedge 2} \iota^{* \wedge 2}=\left(\jmath^{*} \iota^{*}\right)^{\wedge 2}$ is multiplication by $s^{2}$, so $\jmath^{* \wedge 2} \iota^{* \wedge 2}\left(\frac{1}{s} \boldsymbol{b}\right)=s^{2}\left(\frac{1}{s} \boldsymbol{b}\right)=s \boldsymbol{b}$, as desired. Therefore $\operatorname{cor}(\boldsymbol{a})=\jmath^{* \wedge 2}\left(\frac{1}{s} \boldsymbol{a}\right)$.

Now to apply this to $\boldsymbol{a}=\phi^{*} \wedge_{d} \psi^{*} \in \operatorname{Alt}(H, \mathbb{Q} / \mathbb{Z})$, where $\phi^{*}, \psi^{*} \in{ }_{d} H^{*}$. Since $H^{*}$ is divisible, $\phi^{*}$ and $\psi^{*}$ both have $s$-th roots $\frac{1}{s} \phi^{*}$ and $\frac{1}{s} \psi^{*}$. Since $s \nu_{s d}=\nu_{d} \cdot(s \otimes s)$ by $(2.3 .1)$,

$$
\phi^{*} \wedge_{d} \psi^{*}=s\left(\left(\frac{1}{s} \phi^{*}\right) \wedge_{s d}\left(\frac{1}{s} \psi^{*}\right)\right) \in \operatorname{Alt}(H, \mathbb{Q} / \mathbb{Z}) .
$$

Therefore $\frac{1}{s} \phi^{*} \wedge_{s d} \frac{1}{s} \psi^{*}$ is an $s$-th root of $\boldsymbol{a}$ in $\operatorname{Alt}(H, \mathbb{Q} / \mathbb{Z})$. Thus

$$
\operatorname{cor}(\boldsymbol{a})=\jmath^{* \wedge 2}\left(\frac{1}{s} \phi^{*} \wedge_{s d} \frac{1}{s} \psi^{*}\right)=\jmath^{*}\left(\frac{1}{s} \phi^{*}\right) \wedge_{s d} \jmath^{*}\left(\frac{1}{s} \psi^{*}\right) .
$$

This completes the proof.

Remarks 2.11. a. Theorem 2.10 can be used to compute the corestriction for $\iota: H \rightarrow G$ an open embedding of arbitrary proabelian groups, not just those that are torsion-free. For let $\widehat{G}^{*}$ be the divisible hull of $G^{*}$, and let $\widehat{G}$ be the torsion-free proabelian dual of $\widehat{G}^{*}$. Since $G^{*}$ is a subgroup of $\widehat{G}^{*}, G$ is a quotient of $\widehat{G}$, and thus there is a surjective homomorphism $\widehat{G} \rightarrow \bar{G} \simeq G / H$. Let $\widehat{H}$ be the kernel; then $\widehat{H}$ is torsion-free, and $\widehat{H}$ maps onto $H \leq G$. By inflation, the cohomology classes and alternating forms for $H$ and $G$ embed into the corresponding groups for the torsion-free $\widehat{H}$ and $\widehat{G}$, and Theorem 2.10 computes the corestriction.

b. The corestriction map in Theorem 2.10 is not well defined on $\operatorname{Bil}(H, \mathbb{Q} / \mathbb{Z})$. For example, suppose $G=\widehat{\mathbb{Z}} \times \widehat{\mathbb{Z}}$ and $H=2 \widehat{\mathbb{Z}} \times \widehat{\mathbb{Z}}$, so $s=2$. If $\boldsymbol{a} \in \operatorname{Bil}(H, \mathbb{Q} / \mathbb{Z})$, then the choices for $\frac{1}{2} \boldsymbol{a}$ differ by elements of order 2 . But if $\boldsymbol{b} \in \operatorname{Bil}(G, \mathbb{Q} / \mathbb{Z})$ is defined by $\boldsymbol{b}\left(\phi_{i}, \phi_{j}\right)=\delta_{i 1} \delta_{j 1} / 8$, then $\boldsymbol{c}=\operatorname{res}(\boldsymbol{b})$ has order 2 , but $\jmath^{* \otimes 2}(\boldsymbol{c})=\jmath^{* \otimes 2} \iota^{* \otimes 2}(\boldsymbol{b})=$ $s^{2} \boldsymbol{b}=4 \boldsymbol{b} \neq 0$. Therefore $\jmath^{* \otimes 2}\left(\frac{1}{2} \boldsymbol{a}\right) \neq \jmath^{* \otimes 2}\left(\frac{1}{2} \boldsymbol{a}+\boldsymbol{c}\right)$. This shows that in general the value of $\jmath^{* \otimes 2}\left(\frac{1}{s} \boldsymbol{a}\right)$ depends on the choice of $\frac{1}{s} \boldsymbol{a}$.

\section{The IndeX OF AN ALTERNATing FORM}

The index of an alternating form over $\mathbb{Q} / \mathbb{Z}$ is an invariant closely associated with the usual pfaffian of an alternating matrix over a commutative ring. It is related to the (Schur) index of a Brauer class, which is the square root of the dimension of the class's representative division algebra. More precisely, if $F$ is a field of characteristic zero, $G$ is the Galois group of a Galois field extension $\Omega / F$, and $\mu$ is the torsion subgroup of $\Omega^{\times}$, then there is a natural map $\mathrm{H}^{2}(G, \mu) \rightarrow \mathrm{H}^{2}\left(G, \Omega^{\times}\right) \simeq \operatorname{Br}(\Omega / F)$. When the action of $G$ on $\mu$ is trivial, $\mathrm{H}^{2}(G, \mu)$ is isomorphic to $\mathrm{H}^{2}(G, \mathbb{Q} / \mathbb{Z})$, and by Theorem 2.4 there is an induced map $\operatorname{Alt}(G, \mathbb{Q} / \mathbb{Z}) \rightarrow \operatorname{Br}(\Omega / F)$. It will be shown in Theorem 4.10 below that the index of an alternating form is divisible by the index of its image in $\operatorname{Br}(\Omega / F)$.

Definition 3.1. Suppose $G$ is a proabelian group, and $\boldsymbol{a} \in \operatorname{Alt}(G, \mathbb{Q} / \mathbb{Z})$. Define the exponent, degree, and index of $\boldsymbol{a}$ to be the numbers

$$
\exp (\boldsymbol{a})=\exp \left(G / \operatorname{rad}_{\boldsymbol{a}}(G)\right), \quad \operatorname{deg}(\boldsymbol{a})=\left|G / \operatorname{rad}_{\boldsymbol{a}}(G)\right|, \quad \operatorname{ind}(\boldsymbol{a})=\left|G / \operatorname{rad}_{\boldsymbol{a}}(G)\right|^{1 / 2} .
$$

Since it is open in $G, \operatorname{rad}_{\boldsymbol{a}}(G)$ has finite index in $G$, so $\exp (\boldsymbol{a}), \operatorname{deg}(\boldsymbol{a})$, and $\operatorname{ind}(\boldsymbol{a})$ are well defined. Clearly $\exp (\boldsymbol{a})=|\boldsymbol{a}|$, its order in $\operatorname{Alt}(G, \mathbb{Q} / \mathbb{Z})$. It will be shown presently that $\operatorname{ind}(\boldsymbol{a}) \in \mathbb{N}$; it follows immediately that $\exp (\boldsymbol{a})$ and $\operatorname{ind}(\boldsymbol{a})$ 
have the same prime divisors. Later, it will be shown that the index and exponent of an alternating form on an abelian Galois group over a field containing all roots of unity are closely related to the index and exponent of a corresponding Brauer class over the same field.

Definition 3.2. Suppose $G$ is a proabelian group, and $\boldsymbol{b} \in \operatorname{Bil}(G, \mathbb{Q} / \mathbb{Z})$. Define a matrix representing $\boldsymbol{b}$ to be the matrix $\mathcal{B} \in \mathrm{M}_{n}(\mathbb{Q} / \mathbb{Z})$ determined by $\boldsymbol{b}$ with respect to a basis $\left\{\phi_{1}, \ldots, \phi_{n}\right\}$ of the finite abelian group $G / \operatorname{rad}_{\boldsymbol{b}}(G)$. Explicitly, if $b_{i j}=\boldsymbol{b}\left(\phi_{i}, \phi_{j}\right)$, then $\mathcal{B}=\left(b_{i j}\right) \in \mathrm{M}_{n}(\mathbb{Q} / \mathbb{Z})$. Define the row subgroup row $(\boldsymbol{b})$ to be the subgroup of $(\mathbb{Q} / \mathbb{Z})^{n}$ generated by $\mathcal{B}$ 's rows, up to isomorphism.

Note that if $\boldsymbol{a} \in \operatorname{Alt}(G, \mathbb{Q} / \mathbb{Z})$, then $\left(a_{i j}\right) \in \operatorname{Alt}_{n}(\mathbb{Q} / \mathbb{Z})$ is an alternating matrix, with $a_{i j}=\boldsymbol{a}\left(\phi_{i}, \phi_{j}\right)=-a_{j i}$ and $a_{i i}=0$. Theorem 3.6 below shows how to compute the index of an alternating form $\boldsymbol{a}$ directly from any alternating matrix representing $\boldsymbol{a}$. The key observation is given by the next result. The lemma that follows is the basis for later results on the standard form of alternating forms and Brauer classes (Theorem 4.9, Theorem 5.12, and Theorem 5.17).

Proposition 3.3. Suppose $G$ is a proabelian group, and $\boldsymbol{a} \in \operatorname{Alt}(G, \mathbb{Q} / \mathbb{Z})$. Then $\operatorname{row}(\boldsymbol{a})$ is well defined, and it is isomorphic to $G / \operatorname{rad}_{\boldsymbol{a}}(G)$.

Proof. It suffices to show that $\operatorname{row}(\mathcal{A}) \simeq G / \operatorname{rad}_{\boldsymbol{a}}(G)$ for an arbitrary matrix representative $\mathcal{A}$. Let $\bar{\phi}=\left\{\bar{\phi}_{1}, \ldots, \bar{\phi}_{n}\right\}$ be a basis for $G / \operatorname{rad}_{\boldsymbol{a}}(G)$, and let $\mathcal{A} \in \operatorname{Alt}_{n}(\mathbb{Q} / \mathbb{Z})$ be the matrix for $\boldsymbol{a}$ determined by $\bar{\phi}$. Let $r=\exp (\boldsymbol{a})$; then $G / \operatorname{rad}_{\boldsymbol{a}}(G)$ is a quotient of $(\mathbb{Z} / r \mathbb{Z})^{n}$, such that the standard basis $\underline{\phi}=\left\{\phi_{1}, \ldots, \phi_{n}\right\}$ maps to $\bar{\phi}$. By definition of inflation, $\boldsymbol{a}\left(\bar{\phi}_{i}, \bar{\phi}_{j}\right)=\inf (\boldsymbol{a})\left(\phi_{i}, \phi_{j}\right)$, so $\mathcal{A}$ is also the matrix for $\inf (\boldsymbol{a})$ determined by $\phi$. Clearly $G / \operatorname{rad}_{\boldsymbol{a}}(G) \simeq(\mathbb{Z} / r \mathbb{Z})^{n} / \operatorname{rad}_{\inf (\boldsymbol{a})}\left((\mathbb{Z} / r \mathbb{Z})^{n}\right)$, so it now suffices to show $\operatorname{row}(\mathcal{A}) \simeq G / \operatorname{rad}_{\boldsymbol{a}}(G)$ with $G=(\mathbb{Z} / r \mathbb{Z})^{n}$ and $\boldsymbol{a}=\inf (\boldsymbol{a})$. First, a lemma.

Lemma 3.4 "Symplectic Basis I". Suppose $G=(\mathbb{Z} / r \mathbb{Z})^{n}$ and $\boldsymbol{a} \in \operatorname{Alt}(G, \mathbb{Q} / \mathbb{Z})$. Let $\left\{\phi_{1}, \ldots, \phi_{n}\right\}$ be the standard basis for $G$. Then there exists a matrix $\mathcal{P} \in$ $\mathrm{GL}_{n}(\mathbb{Z} / r \mathbb{Z})$ such that $\phi \mathcal{P}=\underline{\psi}$, defined by $\psi_{j}=\prod_{i} \phi_{i}^{p_{i j}}$, is a symplectic basis for $G$ with respect to $\boldsymbol{a}$, so that for some subset $\left\{\psi_{1}, \ldots, \psi_{2 m}\right\} \subset \psi$, along with uniquely determined positive integers $d_{i}: d_{i} \mid d_{i-1}$ and units $w_{i}\left(\bmod \bar{d}_{i}\right)$,

$$
\boldsymbol{a}\left(\psi_{2 i-1}, \psi_{2 i}\right)=-\boldsymbol{a}\left(\psi_{2 i}, \psi_{2 i-1}\right)=w_{i} / d_{i}, \quad 1 \leq i \leq m,
$$

and $\boldsymbol{a}\left(\psi_{k}, \psi_{l}\right)=0$ otherwise. The $w_{i}$ may be arbitrarily designated. If $\mathcal{A} \in$ $\operatorname{Alt}_{n}(\mathbb{Q} / \mathbb{Z})$ is the matrix for a with respect to $\phi$, then $\mathcal{B}=\mathcal{P}^{*} \mathcal{A} \mathcal{P}$ is the alternating 2-block-diagonal matrix for a determined by $\underline{\underline{ }}$.

Proof. By [B, Lemma 1.10], there exists $\mathcal{P}^{\prime} \in \mathrm{GL}_{n}(\mathbb{Z})$ such that if $\underline{\psi}^{\prime}=\phi \mathcal{P}^{\prime}$, then for some subset $\left\{\psi_{1}^{\prime}, \ldots, \psi_{2 m}^{\prime}\right\}, \boldsymbol{a}\left(\psi_{2 i-1}^{\prime}, \psi_{2 i}^{\prime}\right)=-\boldsymbol{a}\left(\psi_{2 i}^{\prime}, \psi_{2 i-1}^{\prime}\right)=u_{i} / d_{i}$ for $i: 1 \leq i \leq m$, where $d_{i} \mid d_{i-1}$, and $u_{i}$ is a unit $\left(\bmod d_{i}\right)$, and $\boldsymbol{a}\left(\psi_{k}^{\prime}, \psi_{l}^{\prime}\right)=0$ otherwise. The proof of this result is similar to the standard construction of a symplectic basis over a vector space. Since $G$ is a free $\mathbb{Z} / r \mathbb{Z}$-module, $\underline{\psi}^{\prime}$ is a basis for $G$. Let $\mathcal{P}_{r}^{\prime} \in \mathrm{GL}_{n}(\mathbb{Z} / r \mathbb{Z})$ be the reduction of $\mathcal{P}^{\prime}(\bmod r)$.

Let $w_{i}$ be any unit $\left(\bmod d_{i}\right)$. Then $w_{i} / d_{i}$ and $u_{i} / d_{i}$ generate the same subgroup of $\mathbb{Q} / \mathbb{Z}$, so $v_{i}^{\prime}\left(u_{i} / d_{i}\right)=w_{i} / d_{i}$ for some $v_{i}^{\prime} \in\left(\mathbb{Z} / d_{i} \mathbb{Z}\right)^{\times}$. Choose $v_{i} \in(\mathbb{Z} / r \mathbb{Z})^{\times}$such that $v_{i} \equiv v_{i}^{\prime}\left(\bmod d_{i}\right)$, which is possible since $d_{i} \mid r$. Let $\mathcal{D} \in \mathrm{GL}_{n}(\mathbb{Z} / r \mathbb{Z})$ be the diagonal matrix with $(2 i-1,2 i-1)$-entry $v_{i}, i=1, \ldots, m$, and the other diagonal entries 1. Let $\underline{\psi}=\underline{\psi^{\prime}} \mathcal{D}$. Then $\boldsymbol{a}\left(\psi_{2 i-1}, \psi_{2 i}\right)=\boldsymbol{a}\left(v_{i} \psi_{2 i-1}^{\prime}, \psi_{2 i}^{\prime}\right)=v_{i} u_{i} / d_{i}=w_{i} / d_{i}$. Let $\mathcal{P}=\mathcal{P}_{r}^{\prime} \mathcal{D}$; then the desired symplectic basis is $\underline{\psi}=\underline{\phi}$, with $\mathcal{P} \in \mathrm{GL}_{n}(\mathbb{Z} / r \mathbb{Z})$. 
It is now obvious that $\operatorname{rad}_{\boldsymbol{a}}(G) \simeq \coprod_{i=1}^{m} d_{i} \mathbb{Z} \times d_{i} \mathbb{Z}$, hence

$$
G / \operatorname{rad}_{\boldsymbol{a}}(G) \simeq \coprod_{i=1}^{m}\left(\mathbb{Z} / d_{i} \mathbb{Z} \times \mathbb{Z} / d_{i} \mathbb{Z}\right) .
$$

It follows that the $d_{i}$ are uniquely determined, as the abelian group invariants of $G / \operatorname{rad}_{\boldsymbol{a}}(G)$. Finally, applying the bilinear relations to $\boldsymbol{a}\left(\prod_{k} \phi^{p_{k i}}, \prod_{l} \phi_{l}^{p_{l j}}\right)$ yields

$$
\boldsymbol{a}\left(\psi_{i}, \psi_{j}\right)=\sum_{1 \leq k<l \leq n}\left(p_{k i} p_{l j}-p_{k j} p_{l i}\right) a_{k l},
$$

the $i j$-th entry of $\mathcal{P}^{*} \mathcal{A P}$. This proves the lemma.

To finish the proof of the proposition, let $\mathcal{B}=\mathcal{P}^{*} \mathcal{A P}$ be the matrix for $\boldsymbol{a}$ determined by $\underline{\psi}$ in Lemma 3.4 , with $w_{i}=1$. Then $\mathcal{B}$ is a direct sum of $m$ alternating $2 \times 2$ blocks, whose nonzero entries are $\pm 1 / d_{i}$. Evidently $\operatorname{row}(\mathcal{B}) \simeq G / \operatorname{rad}_{\boldsymbol{a}}(G)$. Since $\mathcal{P}$ is invertible, $\operatorname{row}(\mathcal{A}) \simeq \operatorname{row}(\mathcal{B})$. This completes the proof.

The determinant of an alternating matrix over a commutative ring is zero if the matrix has odd degree, and it is a square if the matrix has even degree. The positive square root is called the pfaffian of the matrix. The naïve computation of determinants over $\mathbb{Q} / \mathbb{Z}$ is not well defined, but the definition can be adjusted to make sense, as follows.

A degree $m$ submatrix $\mathcal{M} \subset \mathcal{A}$ of an alternating matrix $\mathcal{A}=\left(a_{i j}\right) \in \operatorname{Alt}_{n}(\mathbb{Q} / \mathbb{Z})$ is a matrix obtained by intersecting $m$ rows $i_{1}, \ldots, i_{m}$ with $m$ columns $j_{1}, \ldots, j_{m}$. A degree $m$ minor is the determinant of a degree $m$ submatrix. A degree $m a l$ ternating submatrix $\mathcal{S} \subset \mathcal{A}$ is obtained by intersecting $m$ rows $i_{1}, \ldots, i_{m}$ with the corresponding set of $m$ columns $i_{1}, \ldots, i_{m}$.

Definition 3.5. Suppose $G$ is a proabelian group, and $\boldsymbol{a} \in \operatorname{Alt}(G, \mathbb{Q} / \mathbb{Z})$. The minor subgroup $\operatorname{mr}(\boldsymbol{a})$ is the subgroup of $\mathbb{Q} / \mathbb{Z}$ generated by all of the minors of any matrix $\mathcal{A}$ representing $\boldsymbol{a}$, computed naïvely. The pfaffian subgroup $\operatorname{pf}(\boldsymbol{a})$ is the subgroup of $\mathbb{Q} / \mathbb{Z}$ generated by the pfaffians of all even-degree alternating submatrices of $\mathcal{A}$, computed naïvely. Thus

$$
\begin{aligned}
\operatorname{mr}(\boldsymbol{a}) & =\langle\operatorname{mr}(\mathcal{M}): \mathcal{N} \subset \mathcal{A} \text { submatrix }\rangle \leq \mathbb{Q} / \mathbb{Z}, \\
\operatorname{pf}(\boldsymbol{a}) & =\langle\operatorname{pf}(\mathcal{S}): S \subset \mathcal{A} \text { alternating, even degree }\rangle \leq \mathbb{Q} / \mathbb{Z}
\end{aligned}
$$

"Computed naïvely" means computed from an arbitrary representative of $\boldsymbol{a}$ in $\mathrm{M}_{n}(\mathbb{Q})$, then interpreted $\bmod \mathbb{Z}$. Both $\operatorname{mr}(\boldsymbol{a})$ and $\operatorname{pf}(\boldsymbol{a})$ are well defined. For by B. Proposition 2.3, Proposition 2.8], $|\operatorname{row}(\mathcal{A})|=|\operatorname{mr}(\mathcal{A})|=|\operatorname{pf}(\mathcal{A})|^{2}$ for any matrix $\mathcal{A}$ representing $\boldsymbol{a}$. Therefore, $\operatorname{since} \operatorname{row}(\boldsymbol{a})$ is well defined by Proposition 3.3,

$$
|\operatorname{row}(\boldsymbol{a})|=|\operatorname{mr}(\boldsymbol{a})|=|\operatorname{pf}(\boldsymbol{a})|^{2} .
$$

Since a finite subgroup of $\mathbb{Q} / \mathbb{Z}$ is determined by its order, $\operatorname{mr}(\boldsymbol{a})$ and $\operatorname{pf}(\boldsymbol{a})$ are well defined. Note that if $\mathcal{A}$ has degree $n$, there are $\sum_{i=1}^{n}\left(\begin{array}{c}n \\ i\end{array}\right)^{2}$ submatrices, but a mere $\sum_{i=1}^{\lfloor n / 2\rfloor}\left(\begin{array}{c}n \\ 2 i\end{array}\right)$ alternating, even degree submatrices.

The following theorem is immediate.

Theorem 3.6 "Index Formula". Suppose $G$ is a proabelian group, and $\boldsymbol{a} \in$ $\operatorname{Alt}(G, \mathbb{Q} / \mathbb{Z})$. Then

$$
\operatorname{ind}(\boldsymbol{a})=|\operatorname{pf}(\boldsymbol{a})| \text {. }
$$


Proof. By Proposition 3.3, $|\operatorname{row}(\boldsymbol{a})|=\operatorname{deg}(\boldsymbol{a})$. Since $|\operatorname{row}(\boldsymbol{a})|=|\operatorname{pf}(\boldsymbol{a})|^{2},|\operatorname{pf}(\boldsymbol{a})|=$ ind $(\boldsymbol{a})$ by Definition 3.1.

Example 3.7. Suppose $G=\widehat{\mathbb{Z}}^{n}$. Any matrix $\mathcal{A}$ representing $\boldsymbol{a} \in \operatorname{Alt}(G, \mathbb{Q} / \mathbb{Z})$ may be viewed by inflation as an element of $\operatorname{Alt}_{n}(\mathbb{Q} / \mathbb{Z})$. If $n=2, \mathcal{A}=\left(\begin{array}{cc}0 & a_{12} \\ -a_{12} & 0\end{array}\right)$ for some $a_{12} \in \mathbb{Q} / \mathbb{Z}$. Thus $\operatorname{ind}(\boldsymbol{a})=\exp (\boldsymbol{a})=\left|a_{12}\right|$ in this case. If $n=3$, $\operatorname{ind}(\boldsymbol{a})=\exp (\boldsymbol{a})=\operatorname{lcm}\left[\left|a_{12}\right|,\left|a_{13}\right|,\left|a_{23}\right|\right]$, since $\operatorname{pf}(\mathcal{A})$ is defined with respect to $\mathcal{A}$ 's even degree (alternating) submatrices. If $n=4$,

$$
\mathcal{A}=\left(\begin{array}{cccc}
0 & a_{12} & a_{13} & a_{14} \\
-a_{12} & 0 & a_{23} & a_{24} \\
-a_{13} & -a_{23} & 0 & a_{34} \\
-a_{14} & -a_{24} & -a_{34} & 0
\end{array}\right)
$$

Compute $\exp (\boldsymbol{a})=\operatorname{lcm}\left[\left|a_{12}\right|,\left|a_{13}\right|,\left|a_{14}\right|,\left|a_{23}\right|,\left|a_{24}\right|,\left|a_{34}\right|\right]$ and

$$
\operatorname{ind}(\boldsymbol{a})=\operatorname{lcm}\left[\exp (\boldsymbol{a}),\left|a_{12} a_{34}-a_{13} a_{24}+a_{14} a_{23}\right|\right] .
$$

This is the first case where index is not necessarily equal to exponent. The formula for a degree 6 pfaffian of a $6 \times 6$ alternating matrix $\left(a_{i j}\right)$ is

$$
\begin{aligned}
& a_{12} a_{34} a_{56}-a_{12} a_{35} a_{46}+a_{12} a_{36} a_{45}-a_{13} a_{24} a_{56}+a_{13} a_{25} a_{46}-a_{13} a_{26} a_{45} \\
& +a_{14} a_{23} a_{56}-a_{14} a_{25} a_{36}+a_{14} a_{26} a_{35}-a_{15} a_{23} a_{46}+a_{15} a_{24} a_{36}-a_{15} a_{26} a_{34} \\
& +a_{16} a_{23} a_{45}-a_{16} a_{24} a_{35}+a_{16} a_{25} a_{34} .
\end{aligned}
$$

Lemma 3.4 will be needed for the next section in a slightly more general form.

Lemma 3.8 "Symplectic Basis II". Suppose $\boldsymbol{a} \in \operatorname{Alt}(G, \mathbb{Q} / \mathbb{Z})$, where $G$ is a finite abelian group that has homogeneous primary components. Then $G$ has a basis $\underline{\psi}=\left\{\psi_{i}\right\}_{I}$ that is symplectic with respect to $\boldsymbol{a}$, so that for some subset $\left\{\psi_{1}, \ldots, \bar{\psi}_{2 m}\right\} \subset \underline{\psi}$, along with uniquely determined numbers $d_{i}: d_{i} \mid d_{i-1}$,

$$
\boldsymbol{a}\left(\psi_{2 i-1}, \psi_{2 i}\right)=-\boldsymbol{a}\left(\psi_{2 i}, \psi_{2 i-1}\right)=1 / d_{i}, \quad 1 \leq i \leq m,
$$

and $\boldsymbol{a}\left(\psi_{k}, \psi_{l}\right)=0$ otherwise.

Proof. Let $p_{1}, \ldots, p_{t}$ denote the prime support of $r=\exp (\boldsymbol{a})$. Let $\mathrm{G}_{h}$ denote $G$ 's (homogeneous) $p_{h}$-component, and let $\boldsymbol{a}_{h}$ denote $\boldsymbol{a}$ 's $p_{h}$-component, so $\boldsymbol{a}=$ $\sum_{h=1}^{t} \boldsymbol{a}_{h}$. Since $\boldsymbol{a}_{h}$ is supported on $\mathrm{G}_{h}$ and $\mathrm{G}_{h}$ is homogeneous, by Lemma $3.4 \mathrm{G}_{h}$ has a symplectic basis $\underline{\phi}_{h}=\left\{\phi_{h i}\right\}_{I_{h}}$ for some index set $I_{h}$, such that for some subset $\left\{\phi_{h 1}, \ldots, \phi_{h\left(2 m_{h}\right)}\right\} \subset \underline{\phi}_{h}$, along with uniquely determined numbers $e_{h i}: e_{h i} \leq e_{h i-1}$ and selectable units $w_{h i}\left(\bmod p_{h}^{e_{h i}}\right)$,

$$
\boldsymbol{a}_{h}\left(\phi_{h 2 i-1}, \phi_{h 2 i}\right)=-\boldsymbol{a}_{h}\left(\phi_{h 2 i}, \phi_{h 2 i-1}\right)=w_{h i} / p_{h}^{e_{h i}}, \quad 1 \leq i \leq m_{h},
$$

and $\boldsymbol{a}_{h}\left(\phi_{h k}, \phi_{h l}\right)=0$ otherwise. The problem now is to combine these bases.

Let $m=\max \left\{m_{h}\right\}$, let $H_{i}=\left\{h: i \leq m_{h}\right\}$, and for fixed $i \leq m$, let $d_{i}=$ $\prod_{H_{i}} p_{h}^{e_{h i}}$. Then since $e_{h i} \leq e_{h i-1}$ for each $h, d_{i}$ divides $d_{i-1}$. Choose the $w_{h i}$ so that $\sum_{H_{i}} w_{h i} / p_{h}^{e_{h i}}=1 / d_{i}(\bmod \mathbb{Z})$, using the standard "gcd identity". For $i=1, \ldots, m$, let $\psi_{2 i-1}=\prod_{H_{i}} \phi_{h 2 i-1}$ and $\psi_{2 i}=\prod_{H_{i}} \phi_{h 2 i}$. Extend $\left\{\psi_{1}, \ldots, \psi_{2 m}\right\}$ to a basis $\underline{\psi}$ 
for $G$. Compute, for $i: 1 \leq i \leq m$,

$$
\begin{aligned}
\boldsymbol{a}\left(\psi_{2 i-1}, \psi_{2 i}\right) & =\sum_{h=1}^{t} \boldsymbol{a}_{h}\left(\psi_{2 i-1}, \psi_{2 i}\right)=\sum_{h \in H_{i}} \boldsymbol{a}_{h}\left(\phi_{h 2 i-1}, \phi_{h 2 i}\right)=\sum_{h \in H_{i}} w_{h i} / p_{h}^{e_{h i}} \\
& =1 / d_{i}=-\boldsymbol{a}\left(\psi_{2 i}, \psi_{2 i-1}\right) .
\end{aligned}
$$

Clearly $\boldsymbol{a}\left(\psi_{k}, \psi_{l}\right)=0$ in all other cases, since all of $\boldsymbol{a}$ 's support is spanned by the subset $\left\{\psi_{1}, \ldots, \psi_{2 m}\right\}$.

Any symplectic basis for $G$ with respect to $\boldsymbol{a}$ decomposes into primary components to yield symplectic bases for each $\boldsymbol{a}_{h}$, and its invariants decompose accordingly into invariants $p_{h}^{e_{h i}}$. Since by Lemma 3.4 these are uniquely determined for $\boldsymbol{a}_{h}$, the $d_{i}$ are uniquely determined for $\boldsymbol{a}$. This completes the proof.

Remark 3.9. Lemma 3.8 shows that whenever the finite abelian group $G$ has homogeneous primary components, $G$ has a basis with respect to which $\boldsymbol{a}$ is an alternating 2-block-diagonal matrix, with coefficients $1 / d_{i}$ in $\mathbb{Q} / \mathbb{Z}$. This matrix will be called a canonical form for $\boldsymbol{a}$. Suppose $G$ has exponent $d$. If $\underline{\psi}=\left\{\psi_{1}, \ldots, \psi_{2 m}\right\}$ is a symplectic basis, let $\psi^{*}=\left\{\psi_{1}^{*}, \ldots, \psi_{2 m}^{*}\right\}$ denote the $d u a l$ basis for $G^{*}={ }_{d} G^{*}$, as in [L, Chapter 1, Section 1], defined by $\psi_{i}^{*}\left(\psi_{j}\right)=\delta_{i j} /\left|\psi_{i}\right|$. Let $n_{i}=\left|\psi_{i}^{*}\right|$, and set $d_{i}=\operatorname{gcd}\left(n_{2 i-1}, n_{2 i}\right)$. Then

$$
\boldsymbol{a}=\sum_{i=1}^{m}\left(\frac{n_{2 i-1}}{d_{i}} \psi_{2 i-1}^{*}\right) \wedge_{d_{i}}\left(\frac{n_{2 i}}{d_{i}} \psi_{2 i}^{*}\right) .
$$

This expression will also be called a canonical form for $\boldsymbol{a}$.

\section{Transport to $\operatorname{Br}(F)$}

In this section all of the results on alternating forms, including index, standard form, and functoriality, are transported to the Brauer group.

Setup 4.1. Let $F$ be a field of characteristic 0 that contains all roots of unity $\mu$. Let $F_{\mathrm{ab}}$ denote $F$ 's maximal abelian extension. Assume $F_{\mathrm{ab}} / F$ is nontrivial; then since $\mu \subset F, \mathrm{G}_{F}^{\mathrm{ab}}=\mathrm{G}_{F_{\mathrm{ab}} / F}$ is torsion-free. Let

$$
\omega: \mathbb{Q} / \mathbb{Z} \stackrel{\sim}{\longrightarrow} \mu
$$

denote a fixed isomorphism. Since $\mu \subset F$, this is a $\mathrm{G}_{F}^{\mathrm{ab}}$-map. Set $\omega_{d}^{a}=\omega(a / d)$ and $\omega_{d}=\omega_{d}^{1}$. Note that any other choice of isomorphism $\omega^{\prime}: \mathbb{Q} / \mathbb{Z} \rightarrow \mu$ differs from $\omega$ by a constant $u \in \widehat{\mathbb{Z}}^{\times}$, so that $\omega_{d}^{\prime}=\omega_{d}^{u}$, where $u$ is interpreted $(\bmod d)$. Write $\omega^{\prime}=\omega^{u}$ in this case. Let $\omega_{*}$ denote any of the isomorphisms $\mathcal{F}(-, \mathbb{Q} / \mathbb{Z}) \rightarrow \mathcal{F}(-, \mu)$, where $\mathcal{F}=$ Hom, Bil, $\mathrm{H}^{2}$, or Alt, induced by $\omega$. Let

$$
1 \longrightarrow \mathrm{G}_{\Omega / K} \stackrel{\iota}{\longrightarrow} \mathrm{G}_{\Omega / F} \stackrel{\pi}{\longrightarrow} \mathrm{G}_{K / F} \longrightarrow 1
$$

be a short exact sequence of abelian Galois groups, where $\exp \left(\mathrm{G}_{K / F}\right)=r \in \mathbb{N}$. View $\mathrm{G}_{\Omega / K}$ as a subgroup of $\mathrm{G}_{\Omega / F}$. For $d: r \mid d$, let

$$
\jmath_{d}: \mathrm{G}_{\Omega / F} \rightarrow \mathrm{G}_{\Omega / K}
$$

denote exponentiation by $d$. If $\iota$ is open, set $s=\left|\mathrm{G}_{K / F}\right|$, and let $\jmath:=\jmath_{s}$ denote the transfer.

For each $d$ there is an isomorphism

$$
\omega_{*}:{ }_{d} \mathrm{G}_{\Omega / F}^{*} \stackrel{\sim}{\longrightarrow} \mathrm{H}^{1}\left(\mathrm{G}_{\Omega / F}, \mu_{d}\right) .
$$


Exponentiation by $d$ produces an exact sequence

$$
1 \rightarrow \mu_{d} \rightarrow \Omega^{\times} \rightarrow \Omega^{\times} \rightarrow 1
$$

which by Hilbert 90 determines a natural isomorphism $\partial d:\left(\Omega^{d} \cap F^{\times}\right) / F^{\times} d \stackrel{\sim}{\longrightarrow}$ $\mathrm{H}^{1}\left(\mathrm{G}_{\Omega / F}, \mu_{d}\right)$. The composition $\kappa_{\omega_{d}}=\partial n \cdot \omega_{*}$ yields the Kummer isomorphism

$$
\kappa_{\omega_{d}}:{ }_{d} \mathrm{G}_{\Omega / F}^{*} \stackrel{\sim}{\longrightarrow}\left(\Omega^{d} \cap F^{\times}\right) / F^{\times d} .
$$

To compute this map, let

$$
\langle-,-\rangle: \mathrm{G}_{\Omega / F} \times\left(\Omega^{d} \cap F^{\times}\right) \longrightarrow \mu_{d}
$$

denote the $d$-Kummer pairing, defined here for all $d \in \mathbb{N}$ by $\langle\sigma, x\rangle_{d}=\sigma\left(x^{1 / d}\right) / x^{1 / d}$, where $x^{1 / d}$ is any root of the polynomial $X^{d}-x$. If $\phi^{*} \in{ }_{d} \mathrm{G}_{\Omega / F}^{*}$ and $\kappa_{\omega_{d}}\left(\phi^{*}\right)=x$, then $\omega_{*} \cdot \phi^{*}=\langle-, x\rangle_{d}$. Say $x=\kappa_{\omega_{d}}\left(\phi^{*}\right)$ is the Kummer element associated to the Kummer character $\phi^{*}$.

By the bilinearity of the Kummer pairing, another choice $\omega^{\prime}=\omega^{u}, u \in \widehat{\mathbb{Z}}^{\times}$, induces a new Kummer isomorphism $\kappa_{\omega_{d}^{\prime}}=\kappa_{\omega_{d}}^{u}$, so that if $\kappa_{\omega_{d}}\left(\phi^{*}\right)=x$, then $\kappa_{\omega_{d}^{\prime}}\left(\phi^{*}\right)=x^{u}$, where $u$ is interpreted $(\bmod d)$.

If $d \mid e$, the inclusion ${ }_{d} \mathrm{G}_{\Omega / F}^{*} \rightarrow{ }_{e} \mathrm{G}_{\Omega / F}^{*}$ induces the map

$$
\begin{aligned}
\left(\Omega^{d} \cap F^{\times}\right) / F^{\times d} & \longrightarrow\left(\Omega^{e} \cap F^{\times}\right) / F^{\times e} \\
x & \longmapsto x^{e / d}
\end{aligned}
$$

since, if $x \in \Omega^{d} \cap F^{\times},\langle-, x\rangle_{d}=\langle-, x\rangle_{e}^{e / d}=\left\langle-, x^{e / d}\right\rangle_{e}$. Thus $\kappa_{\omega_{e}} \cdot \kappa_{\omega_{d}}^{-1}(x)=$ $x^{e / d}\left(\bmod F^{\times e}\right)$.

If $\rho: \mathrm{G}_{\Omega^{\prime} / F^{\prime}} \rightarrow \mathrm{G}_{\Omega / F}$ is a map of abelian Galois groups, define for each $d \in \mathbb{N}$ the Kummer transpose of $\rho$ by the right vertical arrow in the diagram

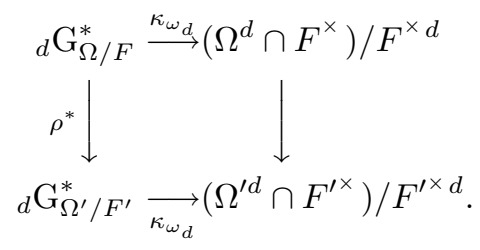

Let $\rho^{*}$ stand for both maps.

If $r \mid d$, then by Lemma 2.7, the sequence of Kummer transpose maps

$$
1 \longrightarrow \frac{K^{d} \cap F^{\times}}{F^{\times d}} \stackrel{\pi^{*}}{\longrightarrow} \frac{\Omega^{d} \cap F^{\times}}{F^{\times} d} \stackrel{\iota^{*}}{\longrightarrow} \frac{\Omega^{d} \cap K^{\times}}{K^{\times}} \stackrel{J_{d}^{*}}{\longrightarrow} \frac{K^{d} \cap F^{\times}}{F^{\times d}}
$$

is exact, and if $\mathrm{G}_{\Omega / F}$ is torsion-free, $\jmath_{d}^{*}$ is surjective. Thus if $\mathrm{G}_{\Omega / F}$ is torsion-free, $\kappa_{\omega_{d}} \cdot \jmath_{d}^{*}\left(\mathrm{G}_{\Omega / K}^{*}\right)$ describes $K / F$ as a Kummer extension. Explicitly, $K=F\left(Z^{1 / d}\right)$, where $Z \leq F^{\times}$is a preimage of $\kappa_{\omega_{d}} \cdot \jmath_{d}^{*}\left(\mathrm{G}_{\Omega / K}^{*}\right)$ in $F^{\times}$.

Lemma 4.2. The Kummer transpose maps $\pi^{*}$ and $\iota^{*}$ in (4.1.3) are induced by the inclusions $K \subset \Omega$ and $F \subset K$, respectively. If $\iota$ is open and $s=\left|\mathrm{G}_{K / F}\right|$, the corestriction

$$
\jmath^{*}=\jmath_{s}^{*}:\left(\Omega^{s} \cap K^{\times}\right) / K^{\times s} \rightarrow\left(K^{s} \cap F^{\times}\right) / F^{\times s}
$$

is induced by the norm $\mathrm{N}_{K / F}: K^{\times} \rightarrow F^{\times}$. 
Proof. For the first map, suppose $\phi^{*} \in{ }_{d} \mathrm{G}_{\Omega / F}^{*}$. Let $x=\kappa_{\omega_{d}}\left(\phi^{*}\right) \in F^{\times} / F^{\times d}$; then by definition $\omega_{*} \cdot \phi^{*}(\sigma)=\langle\sigma, x\rangle_{d}$ for all $\sigma \in \mathrm{G}_{\Omega / F}$. Since $\iota^{*}$ is restriction, $\omega_{*} \cdot \iota^{*}\left(\phi^{*}\right)(\tau)=\langle\tau, x\rangle_{d}$ for $\tau \in \mathrm{G}_{\Omega / K}$. Thus the Kummer element for $\iota^{*}\left(\phi^{*}\right)$ is $x$, viewed as an element of $K^{\times} / K^{\times d}$, hence $\iota^{*}: F^{\times} / F^{\times} d \rightarrow K^{\times} / K^{\times d}$ is the natural map. Similarly, $\pi^{*}$ is the natural injection.

To show $\jmath^{*}$ is induced by the norm, let $\left\{\sigma_{1}, \ldots, \sigma_{s}\right\}$ be a section $\mathrm{G}_{K / F} \rightarrow \mathrm{G}_{\Omega / F}$. Suppose $\phi^{*} \in{ }_{d} \mathrm{G}_{\Omega / K}^{*}$ and $\kappa_{\omega_{d}}\left(\phi^{*}\right)=y \in\left(\Omega^{d} \cap K^{\times}\right) / K^{\times}$. Identify $y$ with a preimage in $\Omega^{d} \cap K^{\times}$; then $\mathrm{N}_{K / F}(y) \in \Omega^{d} \cap F^{\times}$. To prove the result it suffices to show $\mathrm{N}_{K / F}(y) \equiv \kappa_{\omega_{d}} \cdot \jmath^{*}\left(\phi^{*}\right)\left(\bmod F^{\times d}\right)$.

By definition, $\jmath^{*}\left(\phi^{*}\right)(\tau)=\phi^{*}\left(\tau^{s}\right)=\omega_{*}^{-1}\left(\left\langle\tau^{s}, y\right\rangle_{d}\right)$, for all $\tau \in \mathrm{G}_{\Omega / F}$. Observe that $\left\langle\tau^{s}, y\right\rangle_{d}=\langle\tau, w\rangle_{d}$, where $w=\prod_{i=0}^{s-1} \tau^{i}(y) \in \Omega^{\langle\tau\rangle}$. Explicitly,

$$
\frac{\tau^{s}\left(y^{1 / d}\right)}{y^{1 / d}}=\prod_{i=0}^{s-1} \frac{\tau^{i+1}\left(y^{1 / d}\right)}{\tau^{i}\left(y^{1 / d}\right)}=\frac{\tau\left(w^{1 / d}\right)}{w^{1 / d}} .
$$

Let $q$ be the order of $\tau\left(\bmod \mathrm{G}_{\Omega / K}\right)$. Reorder the $\sigma_{i}$ if necessary so that $\sigma_{1}, \ldots, \sigma_{q}$ represent the cosets of $\mathrm{G}_{\Omega / K}$ in $\langle\tau\rangle \mathrm{G}_{\Omega / K}$, and $\sigma_{q}, \sigma_{2 q}, \ldots, \sigma_{s}$ represent the cosets of $\langle\tau\rangle \mathrm{G}_{\Omega / K}$ in $\mathrm{G}_{\Omega / F}$. Since $y$ is in $K$, it is fixed by $\tau^{q}$, hence $w=z^{s / q}$, where

$$
z=\prod_{i=0}^{q-1} \tau^{i}(y)=\prod_{i=1}^{q} \sigma_{i}(y)
$$

Note since $y \in \Omega^{\times d}, z \in \Omega^{\times d}$. Since $\tau$ fixes $z$ and $z \in K^{\times d},\langle\tau, z\rangle_{d}=\left\langle\tau, \sigma_{i}(z)\right\rangle_{d}$, for all $i$. For $\sigma_{i}$ is defined on any $z^{1 / d}$, since $z^{1 / d} \in \Omega$, and so $\sigma_{i}\left(z^{1 / d}\right)$ is a $d$-th root of $\sigma_{i}(z)$, hence by the definition of the Kummer pairing, $\left\langle\tau, \sigma_{i}(z)\right\rangle_{d}=\sigma_{i}\left(\langle\tau, z\rangle_{d}\right)$. Since $\langle\tau, z\rangle_{d} \in \mu \subset F, \sigma_{i}\left(\langle\tau, z\rangle_{d}\right)=\langle\tau, z\rangle_{d}$. It follows that

$$
\left\langle\tau^{s}, y\right\rangle_{d}=\langle\tau, w\rangle_{d}=\langle\tau, z\rangle_{d}^{s / q}=\prod_{j=1}^{s / q}\left\langle\tau, \sigma_{j q}(z)\right\rangle_{d}=\left\langle\tau, \prod_{j=1}^{s / q} \sigma_{j q}(z)\right\rangle_{d} .
$$

Since $\prod_{j=1}^{s / q} \sigma_{j q}(z)=\prod_{i=1}^{s} \sigma_{i}(y)=\mathrm{N}_{K / F}(y)$, this shows

$$
\jmath^{*}\left(\phi^{*}\right)(\tau)=\omega_{*}^{-1}\left\langle\tau, \mathrm{N}_{K / F}(y)\right\rangle_{d} .
$$

Therefore $\kappa_{\omega_{d}} \cdot \jmath^{*}\left(\phi^{*}\right) \equiv \mathrm{N}_{K / F}(y)\left(\bmod F^{\times}\right)$, as desired.

The Kummer transposes $\iota^{*}$ and $\pi^{*}$ are so natural, the notation for them will be suppressed.

Now to apply this to the Brauer group.

Definition 4.3. Define the interior subgroup $\operatorname{Br}^{\circ}(K / F)$ to be the image of the natural map $\varepsilon_{*}: \mathrm{H}^{2}\left(\mathrm{G}_{K / F}, \mu\right) \rightarrow \mathrm{H}^{2}\left(\mathrm{G}_{K / F}, K^{\times}\right)$, induced by the inclusion $\varepsilon: \mu \hookrightarrow$ $K^{\times}$.

Let $d \in \mathbb{N}$, and let $\Omega / F$ be a field extension with group $\mathrm{G}_{\Omega / F}$. It is well known that combining the cup product

$$
{ }_{d} \mathrm{G}_{\Omega / F}^{*} \times \mathrm{H}^{1}\left(\mathrm{G}_{\Omega / F}^{*}, \mu_{d}\right) \longrightarrow \mathrm{H}^{2}\left(\mathrm{G}_{\Omega / F}, \mu_{d}\right)
$$

with the boundary isomorphism $\partial d:\left(\Omega^{d} \cap F^{\times}\right) / F^{\times} \stackrel{\sim}{\longrightarrow} \mathrm{H}^{1}\left(\mathrm{G}_{\Omega / F}, \mu_{d}\right)$ and the natural map $\varepsilon_{*}: \mathrm{H}^{2}\left(\mathrm{G}_{\Omega / F}, \mu_{d}\right) \rightarrow \mathrm{Br}^{\circ}(\Omega / F)$ yields a natural pairing

$$
{ }_{d} \mathrm{G}_{\Omega / F}^{*} \times\left(\Omega^{d} \cap F^{\times}\right) / F^{\times d} \longrightarrow{ }_{d} \operatorname{Br}(\Omega / F),
$$


which takes a pair $\left\{\phi^{*}, y\right\}$ to the class $\left[\left(\phi^{*}, y\right)\right]$ represented by the cyclic algebra of degree $\left|\phi^{*}\right|$

$$
\left(\phi^{*}, y\right):=\sum K Y^{j}: Y^{d}=y ; \quad Y X=\phi(X) Y, \forall X \in K,
$$

where $K / F$ is the cyclic field extension of degree $\left|\phi^{*}\right|$ fixed by $\operatorname{ker}\left(\phi^{*}\right)$, and $\phi \in$ $\mathrm{G}_{\Omega / F}$ is any element such that $\phi^{*}(\phi)=1 /\left|\phi^{*}\right|$. The Kummer isomorphism $\kappa_{\omega_{d}}$ : ${ }_{d} \mathrm{G}_{\Omega / F}^{*} \stackrel{\sim}{\longrightarrow}\left(\Omega^{d} \cap F^{\times}\right) / F^{\times d}$, which is the composition of $\partial d$ and $\omega_{*}$, then yields the antisymmetric bilinear symbol pairing

$$
\left(\Omega^{d} \cap F^{\times}\right) / F^{\times d} \times\left(\Omega^{d} \cap F^{\times}\right) / F^{\times d} \longrightarrow{ }_{d} \operatorname{Br}(\Omega / F),
$$

which takes a pair $\{x, y\}$ to the class $\left[(x, y)_{\omega_{d}}\right]$ represented by the symbol algebra of degree $d$,

$$
(x, y)_{\omega_{d}}=\sum F X^{i} Y^{j}: X^{d}=x ; Y^{d}=y ;[Y, X]=\omega_{d},
$$

where $x$ and $y$ are identified with preimages in $F^{\times}$. By construction, if $\kappa_{\omega_{d}}\left(\phi^{*}\right)=x$, then $\left(\phi^{*}, y\right) \simeq(x, y)_{\omega_{d}}$. Let the symbol subgroup of $\operatorname{Br}(K / F)$ denote the subgroup generated by all symbol classes that are split by $K$.

If $c \in \mathbb{N}$, let $(x, y)_{\omega_{d}}^{c}$ denote either of the (isomorphic) algebras $\left(x, y^{c}\right)_{\omega_{d}}$ or $\left(x^{c}, y\right)_{\omega_{d}}$. By the bilinearity of the bracket, if $(u, d)=1$, then $(x, y)_{\omega_{d}} \simeq\left(x, y^{u}\right)_{\omega_{d}^{u}}$. In particular, if $\omega^{\prime}=\omega^{u}, u \in \widehat{\mathbb{Z}}^{\times}$, is another choice of $\omega$, then $(x, y)_{\omega_{d}} \simeq\left(x, y^{u}\right)_{\omega_{d}^{\prime}}$, where $u$ is interpreted $(\bmod d)$. Note $c\left[(x, y)_{\omega_{d}}\right]=\left[(x, y)_{\omega_{d}}^{c}\right]=\left[(x, y)_{\omega_{d}}^{\otimes c}\right]$. By $[\mathrm{D}$, Section 11, Lemma 6], $c\left[(x, y)_{\omega_{c d}}\right]=\left[(x, y)_{\omega_{d}}\right]$.

Theorem 4.4. Suppose $F$ is a field containing $\mu$, and $\Omega / F$ is a Galois extension with abelian Galois group $\mathrm{G}_{\Omega / F}$. Fix $d \in \mathbb{N}$. Suppose $\phi^{*}, \psi^{*} \in{ }_{d} \mathrm{G}_{\Omega / F}^{*}$. Let $x=$ $\kappa_{\omega_{d}}\left(\phi^{*}\right)$ and $y=\kappa_{\omega_{d}}\left(\psi^{*}\right)$, let $\kappa^{\wedge 2}$ denote the functorial map $\varepsilon_{*} \cdot \omega_{*} \cdot \operatorname{alt}^{-1}$. Then

$$
\begin{aligned}
& \kappa^{\wedge 2}: \operatorname{Alt}\left(\mathrm{G}_{\Omega / F}, \mathbb{Q} / \mathbb{Z}\right) \longrightarrow \operatorname{Br}(\Omega / F), \\
& \phi^{*} \wedge_{d} \psi^{*} \longmapsto\left[(x, y)_{\omega_{d}}\right] .
\end{aligned}
$$

In particular, $\operatorname{Br}^{\circ}(\Omega / F)$ is generated by symbols $\left[(x, y) \omega_{d}\right]$ with $x, y \in \Omega^{d} \cap F^{\times}$, and $\operatorname{Br}^{\circ}\left(F_{\mathrm{ab}} / F\right)$ is the entire symbol subgroup of $\operatorname{Br}(F)$. By the Merkurjev-Suslin theorem, $\operatorname{Br}^{\circ}\left(F_{\mathrm{ab}} / F\right)=\operatorname{Br}(F)$.

Suppose $F^{\prime}$ and $F$ are fields containing $\mu$, and $\rho: \mathrm{G}_{\Omega^{\prime} / F^{\prime}} \rightarrow \mathrm{G}_{\Omega / F}$ is a homomorphism of abelian Galois groups that induces a well-defined homomorphism on Brauer groups. Then the induced map is given by

$$
\begin{aligned}
\rho^{* \wedge 2}: \operatorname{Br}^{\circ}(\Omega / F) & \longrightarrow \operatorname{Br}^{\circ}\left(\Omega^{\prime} / F^{\prime}\right), \\
{\left[(x, y)_{\omega_{d}}\right] } & \longmapsto\left[\left(\rho^{*}(x), \rho^{*}(y)\right)_{\omega_{d}}\right],
\end{aligned}
$$

where $\rho^{*}:\left(\Omega^{d} \cap F^{\times}\right) / F^{\times d} \rightarrow\left(\Omega^{\prime d} \cap F^{\prime \times}\right) / F^{\prime \times d}$ is the Kummer transpose (4.1.2).

Proof. The element $\phi^{*} \otimes_{d} \psi^{*}$ arises from the cup product

$$
{ }_{d} \mathrm{G}_{\Omega / F}^{*} \times{ }_{d} \mathrm{G}_{\Omega / F}^{*} \longrightarrow \mathrm{H}^{2}\left(\mathrm{G}_{\Omega / F}, \mathbb{Q} / \mathbb{Z}\right) .
$$

By functoriality of the cup product, this pairing commutes with the pairing (4.3.1), hence with the symbol pairing (4.3.3). By hypothesis, the map $\kappa_{\omega_{d}}:{ }_{d} \mathrm{G}_{\Omega / F} \stackrel{\sim}{\longrightarrow}$ $\left(\Omega^{d} \cap F^{\times}\right) / F^{\times d}$ sends $\phi^{*}$ to $x$ and $\psi^{*}$ to $y$. Therefore the resulting map $\varepsilon_{*} \cdot \omega_{*}$ : $\mathrm{H}^{2}\left(\mathrm{G}_{\Omega / F}, \mathbb{Q} / \mathbb{Z}\right) \rightarrow \operatorname{Br}^{\circ}(\Omega / F)$ sends $\left[\phi^{*} \otimes_{d} \psi^{*}\right]$ to $\left[(x, y)_{\omega_{d}}\right]$. Since $\left[\phi^{*} \otimes_{d} \psi^{*}\right]=$ $\operatorname{alt}^{-1}\left(\phi^{*} \wedge_{d} \psi^{*}\right)$, this shows $\varepsilon_{*} \cdot \omega_{*} \cdot \operatorname{alt}^{-1}\left(\phi^{*} \wedge_{d} \psi^{*}\right)=\left[(x, y)_{\omega_{d}}\right]$, as desired. Since alt ${ }^{-1}$ is onto, the image of $\kappa^{\wedge 2}$ is the same as the image of $\varepsilon_{*} \cdot \omega_{*}$, namely $\operatorname{Br}^{\circ}(\Omega / F)$. 
Moreover, since by Theorem $2.4 \operatorname{Alt}\left(\mathrm{G}_{\Omega / F}, \mathbb{Q} / \mathbb{Z}\right)$ is generated by the $\left[\phi^{*} \wedge_{d} \psi^{*}\right]$ for various $d \in \mathbb{N}$, it follows that $\operatorname{Br}^{\circ}(\Omega / F)$ is generated by the $\left[(x, y)_{\omega_{d}}\right]$, where $x, y \in \Omega^{d} \cap F^{\times}$.

Any $x, y \in F^{\times}$are in $F_{\mathrm{ab}}^{d} \cap F^{\times}$for some $d$, hence $\operatorname{Br}^{\circ}\left(F_{\mathrm{ab}} / F\right)$ is the entire symbol subgroup of $\operatorname{Br}(F)$. Since $\mu \subset F, \operatorname{Br}^{\circ}\left(F_{\mathrm{ab}} / F\right)=\operatorname{Br}(F)$ by the Merkurjev-Suslin theorem.

It remains to verify the functorial map. By the functoriality of the cup product, $\rho: \mathrm{G}_{\Omega^{\prime} / F^{\prime}} \rightarrow \mathrm{G}_{\Omega / F}$ induces the map $\rho^{* \wedge 2}: \operatorname{Alt}\left(\mathrm{G}_{\Omega / F}, \mathbb{Q} / \mathbb{Z}\right) \rightarrow \operatorname{Alt}\left(\mathrm{G}_{\Omega^{\prime} / F^{\prime}}, \mathbb{Q} / \mathbb{Z}\right)$, which takes an element $\phi^{*} \wedge_{d} \psi^{*}$ to $\rho^{*}\left(\phi^{*}\right) \wedge_{d} \rho^{*}\left(\psi^{*}\right)$. This translates to the functorial map $\left[\rho^{* \otimes 2}\right]: \mathrm{H}^{2}\left(\mathrm{G}_{\Omega / F}, \mu\right) \rightarrow \mathrm{H}^{2}\left(\mathrm{G}_{\Omega^{\prime} / F^{\prime}}, \mu\right)$, which takes $\left[\phi^{*} \otimes_{d} \psi^{*}\right]$ to $\left[\rho^{*}\left(\phi^{*}\right) \otimes_{d}\right.$ $\left.\rho^{*}\left(\psi^{*}\right)\right]$. By the hypothesis that $\rho$ induces a well defined map on Brauer groups, there is a commutative diagram

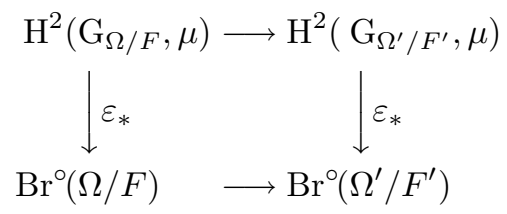

The left vertical arrow sends $\left[\phi^{*} \otimes_{d} \psi^{*}\right]$ to $\left[(x, y)_{\omega_{d}}\right]$, and by $(4.1 .2)$, the right vertical arrow sends $\left[\rho^{*}\left(\phi^{*}\right) \otimes_{d} \rho^{*}\left(\psi^{*}\right)\right]$ to $\left[\left(\rho^{*}(x), \rho^{*}(y)\right)_{\omega_{d}}\right]$. This completes the proof.

Remarks 4.5. a. Since clearly $\omega_{*} \cdot$ alt $^{-1}=\operatorname{alt}^{-1} \cdot \omega_{*}$, the map

$$
\begin{aligned}
\varepsilon_{*} \cdot \operatorname{alt}^{-1}: \operatorname{Alt}\left(\mathrm{G}_{\Omega / F}, \mu\right) & \longrightarrow \operatorname{Br}(\Omega / F) \\
\omega_{*}\left(\phi^{*} \wedge_{d} \psi^{*}\right) & \longmapsto\left[(x, y)_{\omega_{d}}\right]
\end{aligned}
$$

is natural, that is, independent of the choice of $\omega$. Explicitly, suppose $\omega^{\prime}=\omega^{u}$ for some $u \in \widehat{\mathbb{Z}}^{\times}$, and $x^{\prime}=\kappa_{\omega_{d}^{\prime}}\left(\phi^{*}\right), y^{\prime}=\kappa_{\omega_{d}^{\prime}}\left(\psi^{*}\right)$. Then $x^{\prime}=x^{u}$ and $y^{\prime}=y^{u}$, where $u$ is interpreted $(\bmod d)$. Compute $\omega_{*}^{\prime}\left(\phi^{*} \wedge_{d} \psi^{*}\right)=u \omega_{*}\left(\phi^{*} \wedge_{d} \psi^{*}\right)$, and $\left[\left(x^{\prime}, y^{\prime}\right)_{\omega_{d}^{\prime}}\right]=\left[\left(x^{u}, y^{u}\right)_{\omega_{d}^{u}}\right]=u\left[(x, y)_{\omega_{d}}\right]$, as desired.

b. By Theorem 4.4, $\kappa^{\wedge 2}: \operatorname{Alt}\left(\mathrm{G}_{F}^{\mathrm{ab}}, \mathbb{Q} / \mathbb{Z}\right) \rightarrow \operatorname{Br}(F)$ is surjective. However, it might not be injective. For example, if $F=\mathbb{C}(t)$, then $\mathrm{G}_{F}^{\mathrm{ab}}$ is a large nonprocyclic group, so $\operatorname{Alt}\left(\mathrm{G}_{F}^{\mathrm{ab}}, \mu\right)$ is nontrivial. On the other hand, $\operatorname{Br}(F)=\{0\}$ by Tsen's theorem.

The symplectic basis and index results for alternating forms may now be transported to the Brauer group.

Definition 4.6. a. Suppose $\boldsymbol{a} \in \operatorname{Alt}\left(\mathrm{G}_{F}^{\mathrm{ab}}, \mathbb{Q} / \mathbb{Z}\right)$, and $\kappa^{\wedge 2}(\boldsymbol{a})=\boldsymbol{\xi} \in \operatorname{Br}(F)$. Define a matrix representing $\boldsymbol{\xi}$ to be any (alternating) matrix $\left(a_{i j}\right)$ representing $\boldsymbol{a}$, as in Definition 3.2.

b. Suppose $x_{1}, x_{2} \in F^{\times} / F^{\times} d$ and $x_{3}, x_{4} \in F^{\times} / F^{\times e}$ are nontrivial. Call a symbol $\left(x_{1}, x_{2}\right)_{\omega_{d}}$ reduced if the subgroup $\left\langle x_{1}^{1 / d}, x_{2}^{1 / d}\right\rangle \leq\left(F_{\text {ab }}^{\times} / F^{\times}\right)_{\text {tor }}$ has order $d^{2}$. Call a collection of reduced symbols $\left(x_{i}, x_{j}\right)_{\omega_{d_{i j}}}$ disjoint if the subgroup of $\left(F_{\mathrm{ab}}^{\times} / F^{\times}\right)_{\text {tor }}$ generated by all of the $x_{i}^{1 / d_{i j}}, x_{j}^{1 / d_{i j}}$, has order $\prod_{i, j} d_{i j}^{2}$.

c. Call a sum of classes of disjoint reduced symbols in $\operatorname{Br}(F)$ a disjoint reduced symbol presentation.

Example 4.7. Suppose $\left\{x_{1}, \ldots, x_{n}\right\}$ is a subset of $F^{\times}$that is pure independent in $F^{\times} / F^{\times d}$ for some $d \in \mathbb{N}$. For example, the $x_{i}$ could be part of a transcendence 
basis for $F$ over some subfield of $F$. Suppose $\boldsymbol{\xi} \in \operatorname{Br}(F)$ has presentation

$$
\boldsymbol{\xi}=\sum_{1 \leq i<j \leq n}\left[\left(x_{i}, x_{j}\right)_{\omega_{d_{i j}}}^{n_{i j}}\right]
$$

Set $a_{i j}=n_{i j} / d_{i j} \in \mathbb{Q} / \mathbb{Z}$, and set $a_{j i}=-a_{i j}$ if $i<j$. Then $\mathcal{A}=\left(a_{i j}\right)$ is an alternating matrix representing $\boldsymbol{\xi}$. This will be proved in Theorem 4.9. The symbols $\left(x_{i}, x_{j}\right)_{\omega_{d}}$ are reduced, and if $d_{i} \mid d$ for $i=1, \ldots,\lfloor n / 2\rfloor$, then the collection $\left\{\left(x_{2 i-1}, x_{2 i}\right)_{\omega_{d_{i}}}\right\}$ is disjoint.

Remark 4.8. A reduced symbol algebra may be trivial, and disjoint reduced symbol algebras may be isomorphic. For example, when $F=\mathbb{C}(t)$, any symbol algebra is trivial, hence any two symbol algebras of the same degree are isomorphic. On the other hand, Corollary 4.11 gives conditions under which a product of disjoint reduced symbol algebras is a division algebra.

The next theorem shows that it is always possible to express a Brauer class as a sum of disjoint reduced symbol classes. Moreover, it shows how to obtain this expression from a given presentation, provided the parameters of the given symbols are part of a homogeneous set, such as a transcendence base for $F$ over some subfield. More generally, it suffices to have an $\mathfrak{m}$-independent set.

Theorem 4.9 "Disjoint Reduced Symbol Presentation I". Suppose $\boldsymbol{\xi} \in$ $\operatorname{Br}(F)$. For some $\underline{y}=\left\{y_{1}, \ldots, y_{2 m}\right\} \subset F^{\times}$and $d_{i} \mid d_{i-1}$, $\boldsymbol{\xi}$ has a disjoint reduced symbol presentation

$$
\boldsymbol{\xi}=\sum_{i=1}^{m}\left[\left(y_{2 i-1}, y_{2 i}\right)_{\omega_{d_{i}}}\right]
$$

where, as in Definition 4.6, the subgroup of $F_{\mathrm{ab}}^{\times} / F^{\times}$generated by the elements $y_{2 i-1}^{1 / d_{i}}, y_{2 i}^{1 / d_{i}}$ has order $\prod_{i=1}^{n} d_{i}^{2}$.

Homogeneous Case. Suppose $\mathfrak{m} \subset \mathcal{P}, \underline{x}=\left\{x_{1}, \ldots, x_{n}\right\} \subset F^{\times}$is an $\mathfrak{m}$-independent set, and

$$
\boldsymbol{\xi}=\sum_{1 \leq i<j \leq n}\left[\left(x_{i}, x_{j}\right)_{\omega_{r_{i j}}}^{s_{i j}}\right]
$$

where $r_{i j} \in \mathbb{N}(\mathfrak{m})$. Set $a_{i j}=-a_{j i}=s_{i j} / r_{i j}$; then $\left(a_{i j}\right) \in \operatorname{Alt}_{n}(\mathbb{Q} / \mathbb{Z})$ is a matrix that represents $\boldsymbol{\xi}$. Let $r=\operatorname{lcm}\left\{r_{i j}\right\}$. There exists a matrix $\mathcal{P}=\left(p_{i j}\right) \in \mathrm{GL}_{n}(\mathbb{Z} / r \mathbb{Z})$ that puts $\left(a_{i j}\right)$ in standard form, so that $\mathcal{P}^{*}\left(a_{i j}\right) \mathcal{P}=\left(b_{i j}\right)$, where $b_{2 i-12 i}=-b_{2 i 2 i-1}=$ $1 / d_{i}\left(1 \leq i \leq 2 m, d_{i}: d_{i}\left|d_{i-1}\right| r\right)$, and $b_{i j}=0$ otherwise. Let $Q^{*}=\left(q_{i j}^{*}\right)$ be the inverse of $\mathcal{P}^{*}$, and set $\underline{y}=\underline{x} Q^{*}$, so that $\left(\bmod F^{\times r}\right)$,

$$
y_{j}=\coprod_{i=1}^{n} x_{i}^{q_{i j}^{*}}, \quad x_{j}=\coprod_{i=1}^{n} y_{i}^{p_{i j}^{*}} .
$$

Then

$$
\boldsymbol{\xi}=\sum_{i=1}^{m}\left[\left(y_{2 i-1}, y_{2 i}\right)_{\omega_{d_{i}}}\right]
$$

is a disjoint reduced symbol presentation.

Proof. Let $G=\mathrm{G}_{F}^{\mathrm{ab}}$. Let $\boldsymbol{a} \in \operatorname{Alt}(G, \mathbb{Q} / \mathbb{Z})$ be any element such that $\kappa^{\wedge 2}(\boldsymbol{a})=\boldsymbol{\xi}$. Set $r=\exp (\boldsymbol{a})$. Since $G$ is torsion-free, $G / G^{r}$ has homogeneous primary components. Since $G / G^{r}$ is bounded, i.e., of finite exponent, it is a direct product of finite cyclic groups. Since $\boldsymbol{a}$ is continuous, it is supported on a finite direct summand $H / H^{r}$ of $G / G^{r}$. By Lemma 3.8, there exists a symplectic basis for 
$H / H^{r}$ with respect to $\boldsymbol{a}$, such that for some subset $\left\{\psi_{1}, \ldots, \psi_{2 m}\right\}$, and numbers $d_{i}: d_{i}\left|d_{i-1}\right| r, \boldsymbol{a}\left(\psi_{2 i-1}, \psi_{2 i}\right)=-\boldsymbol{a}\left(\psi_{2 i}, \psi_{2 i-1}\right)=1 / d_{i}$, and $\boldsymbol{a}\left(\psi_{k}, \psi_{l}\right)=0$ otherwise. Extend this basis to a basis $\underline{\psi}$ for $G / G^{r}$. Define elements $\left\{\psi_{1}^{*}, \ldots, \psi_{2 m}^{*}\right\} \subset G^{*}$ by $\psi_{i}^{*}\left(\psi_{j}\right)=\delta_{i j} /\left|\psi_{i}^{*}\right|$, for all $i$. Set $m_{i}=\left|\psi_{2 i-1}^{*}\right|, n_{i}=\left|\psi_{2 i}^{*}\right|$, and $r_{i}=\operatorname{gcd}\left(m_{i}, n_{i}\right)$. Since $\left|\boldsymbol{a}\left(\psi_{2 i-1}, \psi_{2 i}\right)\right|=d_{i}, d_{i}$ divides $r_{i}$. Now $\left|\frac{m_{i}}{d_{i}} \psi_{2 i-1}^{*} \wedge_{d_{i}} \frac{n_{i}}{d_{i}} \psi_{2 i}^{*}\right|=d_{i}$ and

$$
\boldsymbol{a}=\sum_{i=1}^{m}\left(\frac{m_{i}}{d_{i}} \psi_{2 i-1}^{*} \wedge_{d_{i}} \frac{n_{i}}{d_{i}} \psi_{2 i}^{*}\right)
$$

For each $d \in \mathbb{N}$, let $\kappa_{\omega_{d}}: G^{*} \rightarrow F_{\mathrm{ab}}^{d} \cap F^{\times} / F^{\times} d$ be the Kummer isomorphism, and set $y_{2 i-1}=\kappa_{\omega_{d_{i}}}\left(\frac{m_{i}}{d_{i}} \psi_{2 i-1}^{*}\right)$ and $y_{2 i}=\kappa_{\omega_{d_{i}}}\left(\frac{n_{i}}{d_{i}} \psi_{2 i}^{*}\right)$. For the statement of the theorem, identify these elements with preimages in $F^{\times}$. By Theorem 4.4,

$$
\kappa^{\wedge 2}\left(\frac{m_{i}}{d_{i}} \psi_{2 i-1}^{*} \wedge_{d_{i}} \frac{n_{i}}{d_{i}} \psi_{2 i}^{*}\right)=\left[\left(y_{2 i-1}, y_{2 i}\right)_{\omega_{d_{i}}}\right] .
$$

Thus $\boldsymbol{\xi}$ has the desired form. Since the characters $\psi_{2 i-1}^{*}$ and $\psi_{2 i}^{*}$ are independent in $G^{*}$, this is a disjoint reduced symbol presentation. This proves the first statement.

Now for the homogeneous case. Assume $\left(s_{i j}, r_{i j}\right)=1$. Let $G$ be the Galois group of $F\left(x_{1}^{1 / r}, \ldots, x_{n}^{1 / r}\right) / F$. Since $\underline{x}$ is $\mathfrak{m}$-independent, $G$ is isomorphic to $(\mathbb{Z} / r \mathbb{Z})^{n}$. Let $\phi=\left\{\phi_{1}, \ldots, \phi_{n}\right\}$ be the basis for $G$ defined by $\left\langle\phi_{i}, x_{j}\right\rangle_{r}=\omega_{r}^{\delta_{i j}}$. Define $\boldsymbol{a} \in$ $\overline{\operatorname{Alt}}(G, \mathbb{Q} / \mathbb{Z})$ by $\boldsymbol{a}\left(\phi_{i}, \phi_{j}\right)=a_{i j}=s_{i j} / r_{i j}$. Since $\left(s_{i j}, r_{i j}\right)=1$, then $r=\operatorname{lcm}\left\{r_{i j}\right\}=$ $\exp (\boldsymbol{a})$. Let $\left\{\phi_{1}^{*}, \ldots, \phi_{n}^{*}\right\}$ be dual to $\underline{\phi}$. Then

$$
\boldsymbol{a}=\sum_{1 \leq i<j \leq n} \frac{r s_{i j}}{r_{i j}}\left(\phi_{i}^{*} \wedge_{r} \phi_{j}^{*}\right)
$$

By Theorem 4.4, $\kappa^{\wedge 2}\left(\phi_{i}^{*} \wedge_{r} \phi_{j}^{*}\right)=\left[\left(x_{i}, x_{j}\right)_{\omega_{r}}\right]$, therefore $\kappa^{\wedge 2}(\boldsymbol{a})=\boldsymbol{\xi}$. Thus $\boldsymbol{a}=\left(a_{i j}\right)$ is a matrix that represents $\boldsymbol{\xi}$. By Lemma 3.4, there exists a basis change $\psi=\phi \mathcal{P}$, where $\underline{\psi}$ is a symplectic basis for $G$ with respect to $\boldsymbol{a}$, and $\mathcal{P} \in \mathrm{GL}_{n}(\mathbb{Z} / r \mathbb{Z})$. Thus for some subset $\left\{\psi_{1}, \ldots, \psi_{2 m}\right\} \subset \psi, \boldsymbol{a}\left(\psi_{2 i-1}, \psi_{2 i}\right)=-\boldsymbol{a}\left(\psi_{2 i}, \psi_{2 i-1}\right)=b_{2 i-12 i}=1 / d_{i}$, and $\boldsymbol{a}\left(\psi_{k}, \psi_{l}\right)=b_{k l}=0$ otherwise, as desired. By Lemma $3.4,\left(b_{i j}\right)=\mathcal{P}^{*}\left(a_{i j}\right) \mathcal{P}$ is the resulting alternating 2-block-diagonal matrix for $\boldsymbol{a}$.

Let $\left\{\psi_{1}^{*}, \ldots, \psi_{2 m}^{*}\right\}$ be part of a basis for $G^{*}$ dual to $\underline{\psi}$, defined by $\psi_{i}^{*}\left(\psi_{j}\right)=\delta_{i j} / r$, for all $i$, and set $y_{i}=\kappa_{\omega_{r}}\left(\psi_{i}^{*}\right) \in F^{\times} / F^{\times r}$. Then $\overline{\boldsymbol{a}}=\sum_{i=1}^{m} \frac{r}{d_{i}}\left(\psi_{2 i-1}^{*} \wedge_{r} \psi_{2 i}^{*}\right)$. By Theorem 4.4, $\kappa^{\wedge 2}\left(\frac{r}{d_{i}}\left(\psi_{2 i-1}^{*} \wedge_{r} \psi_{2 i}^{*}\right)\right)=\left[\left(y_{2 i-1}, y_{2 i}\right)_{\omega_{d_{i}}}\right]$. Thus $\boldsymbol{\xi}$ has the desired form. This is a disjoint reduced symbol presentation since the $\psi_{i}^{*}$ are independent. Since $\underline{\psi}=\underline{\phi} \mathcal{P}$, the transpose is $\underline{\psi}^{*} \mathcal{P}^{*}=\underline{\phi}^{*}$, and by Kummer theory, $\underline{y} \mathcal{P}^{*}=\underline{x}$. If $Q^{*}=\left(\overline{\mathcal{P}}^{*}\right)^{-\overline{1}}$, then $\underline{y}=\underline{x} Q^{*}$. This completes the proof.

The next theorem sets an upper bound for the index of a Brauer class $\boldsymbol{\xi}$ in terms of the index of any alternating form $\boldsymbol{a}$ such that $\kappa^{\wedge 2}(\boldsymbol{a})=\boldsymbol{\xi}$. The goal is to compute an index bound directly from an arbitrary symbol presentation of a given Brauer class. A mere bound is the best that can be expected for the general situation. For example, it follows by Remarks 4.5 that the exponent and index of a Brauer class may be trivial, no matter what the exponent and index of the preimage in $\operatorname{Alt}\left(\mathrm{G}_{F}, \mathbb{Q} / \mathbb{Z}\right)$. On the other hand, Corollary 4.11 below shows the bound is realized over certain fields. 
Theorem 4.10 "Index Bound for $\operatorname{Br}(\boldsymbol{F})$ ". Suppose $\boldsymbol{\xi} \in \operatorname{Br}(F)$. Let $\boldsymbol{a} \in$ $\operatorname{Alt}\left(\mathrm{G}_{F}^{\mathrm{ab}}, \mathbb{Q} / \mathbb{Z}\right)$ be such that $\kappa^{\wedge 2}(\boldsymbol{a})=\boldsymbol{\xi}$. Then

$$
\exp (\boldsymbol{\xi})|\exp (\boldsymbol{a}), \quad \operatorname{ind}(\boldsymbol{\xi})| \operatorname{ind}(\boldsymbol{a})=\operatorname{pf}(\boldsymbol{a}) .
$$

The class $\boldsymbol{\xi}$ is represented by a central simple algebra of degree $\operatorname{pf}(\boldsymbol{a})$.

Proof. Since $\kappa^{\wedge 2}$ is a homomorphism, $\exp (\boldsymbol{\xi})$ divides $\exp (\boldsymbol{a})$. Assume the notation of Theorem 4.9 and its proof. By definition, $\operatorname{ind}(\boldsymbol{a})=\prod d_{i}$, the degree of the algebra $\bigotimes_{i=1}^{m}\left(y_{2 i-1}, y_{2 i}\right)_{\omega_{d_{i}}}$, which represents $\boldsymbol{\xi}$. Since the index of $\boldsymbol{\xi}$ divides the degree of any representative central simple algebra, ind $(\boldsymbol{\xi})$ divides ind $(\boldsymbol{a})$. By the index formula Theorem 3.6, $\operatorname{ind}(\boldsymbol{a})=|\operatorname{pf}(\boldsymbol{a})|$. This completes the proof.

Corollary 4.11. Let $k$ be a field containing $\mu$, and suppose $F=k\left(x_{1}, \ldots, x_{n}\right)$, the rational function field, or $F=k\left(\left(x_{1}\right)\right) \cdots\left(\left(x_{n}\right)\right)$, the iterated power series field, in $n$ variables. Let

$$
A=\bigotimes_{1 \leq i<j \leq n}\left(x_{i}, x_{j}\right)_{\omega_{r_{i j}}}^{s_{i j}}
$$

Then in the situation of Theorem 4.9 (homogeneous case), the division algebra underlying $A$ is

$$
D=\bigotimes_{i=1}^{m}\left(y_{2 i-1}, y_{2 i}\right)_{\omega_{d_{i}}}
$$

Moreover, $\operatorname{ind}(A)=\left|\operatorname{pf}\left(\left(a_{i j}\right)\right)\right|$, where $\left(a_{i j}\right) \in \operatorname{Alt}_{n}(\mathbb{Q} / \mathbb{Z})$ is defined by $a_{i j}=$ $s_{i j} / r_{i j} \in \mathbb{Q} / \mathbb{Z}$.

Proof. Since $k\left(x_{1}, \ldots, x_{n}\right) \subset k\left(\left(x_{1}\right)\right) \cdots\left(\left(x_{n}\right)\right)$, it is enough to prove the result over $F=k\left(\left(x_{1}\right)\right) \cdots\left(\left(x_{n}\right)\right)$. The $x_{i}$ form a pure independent subset of $F^{\times} / F^{\times r}$, where $r=\operatorname{lcm}\left\{r_{i j}\right\}$. By Theorem 4.9, $A \sim D=\bigotimes_{i=1}^{m}\left(y_{2 i-1}, y_{2 i}\right)_{\omega_{d_{i}}}$, for some numbers $m \leq\lfloor n / 2\rfloor$ and $d_{i} \mid r$, and some subset $\underline{y}=\left\{y_{1}, \ldots, y_{n}\right\} \subset F^{\times} / F^{\times r}$, defined by $\underline{y}=\underline{x} Q^{*}$, for some $Q^{*} \in \mathrm{GL}_{n}(\mathbb{Z} / r \mathbb{Z})$. Since $Q^{*}$ is invertible, $\underline{y}$ is a pure independent subset of $F^{\times} / F^{\times r}$. Identify $y$ with any preimage in $F^{\times}$. Since the $d_{i}$ divide $r$, by Kummer theory $L=F\left(\left\{y_{2 i-1}^{1 / d_{i}}, y_{2 i}^{1 / d_{i}}: 1 \leq i \leq m\right\}\right)$ has degree $\prod_{i=1}^{m} d_{i}^{2}$.

Let $v$ denote the standard henselian valuation on $F$ defined by the $x_{i}$, with lexicographically ordered value group $\Gamma_{F} \simeq \mathbb{Z}^{n}$. Since $v$ is henselian, it has a unique extension $v$ to $L$. By multiplicativity, $v\left(y_{2 i-1}^{1 / d_{i}}\right)=\frac{1}{d_{i}} v\left(y_{2 i-1}\right)$ and $v\left(y_{2 i}^{1 / d_{i}}\right)=\frac{1}{d_{i}} v\left(y_{2 i}\right)$. Since $v(\underline{x})$ gives a basis for $\Gamma_{F} / r \Gamma_{F}$, so does $v(\underline{y})$, and it follows easily that the $v\left(y_{2 i-1}^{1 / d_{i}}\right), v\left(y_{2 i}^{1 / d_{i}}\right)$ are independent $\left(\bmod r \Gamma_{F}\right)$, so they give a basis for $\Gamma_{L} / r \Gamma_{F}$. Since $d_{i}$ divides $r$ for all $i$, it then follows easily that $\left|\Gamma_{L} / \Gamma_{F}\right|=\prod d_{i}^{2}=[L: F]$. Therefore $L / F$ is totally ramified.

Claim. Each $\left(y_{2 i-1}, y_{2 i}\right)_{\omega_{d_{i}}}$ is a division algebra. By the norm criterion [R, Corollary 30.7], it is enough to show that if $y_{2 i}^{e}$ is a norm from $K=F\left(y_{2 i-1}^{1 / d_{i}}\right)$, then $d_{i}$ divides $e$. Note that since $K / F$ is totally ramified of degree $d_{i}, \Gamma_{K}=\left\langle\frac{1}{d_{i}} v\left(y_{2 i-1}\right)\right\rangle+$ $\Gamma_{F}$. Since $v$ 's extension to $K$ is uniquely defined, $v(y)=v(\sigma(y))$ for all $\sigma \in \mathrm{G}_{K / F}$ and $y \in K$, hence $v(N(y))=d_{i} v(y)$ and $v\left(N\left(K^{\times}\right)\right)=\left\langle v\left(y_{2 i-1}\right)\right\rangle+d_{i} \Gamma_{F}$. Since $\left\langle v\left(y_{2 i-1}\right)\right\rangle \cap\left\langle v\left(y_{2 i}\right)\right\rangle \subset r \Gamma_{F}$, if $y_{2 i}^{e} \in N\left(K^{\times}\right)$, then $v\left(y_{2 i}^{e}\right) \in d_{i} \Gamma_{F}$, so $d_{i} \mid e$. This proves the claim.

Since $v$ is henselian it has a unique extension from $F$ to each $D_{i}=\left(y_{2 i-1}, y_{2 i}\right)_{\omega_{d_{i}}}$; evidently $\Gamma_{D_{i}}=\left\langle\frac{1}{d_{i}} v\left(y_{2 i-1}\right)\right\rangle+\left\langle\frac{1}{d_{i}} v\left(y_{2 i}\right)\right\rangle+\Gamma_{F} \leq \Gamma_{L}$. By [TW, Extension Lemma 
1.6], since $\Gamma_{D_{i}} \cap \Gamma_{D_{j}}=\Gamma_{F}$ for $i \neq j, D_{i} \otimes_{F} D_{j}$ is a division algebra, and $\Gamma_{D_{i} \otimes_{F} D_{j}}=$ $\Gamma_{D_{i}}+\Gamma_{D_{j}}$. By induction, $D$ is a division algebra.

The last statement is immediate by Theorem 4.10.

The induced map $\rho^{* \wedge 2}$ of Theorem 4.4 is computable in the most interesting cases, namely when $\rho$ is a closed embedding, projection, or the transfer. In these cases, the induced maps on Brauer groups are well defined, and by Theorem 4.4, the computation of $\rho^{* \wedge 2}$ reduces to the computation of the Kummer transpose maps $\rho^{*}$, which was accomplished in Lemma 4.2.

Theorem 4.12. Suppose $K / F$ is a Galois extension with an abelian Galois group $\mathrm{G}_{K / F}$ of finite exponent. Let $d \in \mathbb{N}$. Then

$$
\begin{aligned}
& \text { res : } \operatorname{Br}(F) \rightarrow \operatorname{Br}^{\circ}\left(F_{\mathrm{ab}} / K\right), \quad \quad \text { inf }: \mathrm{Br}^{\circ}(K / F) \hookrightarrow \operatorname{Br}(F), \\
& {\left[(x, y)_{\omega_{d}}\right] \longmapsto\left[(x, y)_{\omega_{d}}\right] \quad\left[(\bar{x}, \bar{y})_{\omega_{d}}\right] \longmapsto\left[(\bar{x}, \bar{y})_{\omega_{d}}\right] .}
\end{aligned}
$$

Suppose $\mathrm{G}_{K / F}$ is finite, of order $s$. For all $x, y \in F_{\mathrm{ab}}^{d} \cap K^{\times}$,

$$
\text { cor: } \begin{aligned}
\operatorname{Br}^{\circ}\left(F_{\mathrm{ab}} / K\right) & \longrightarrow \operatorname{Br}(F) \\
{\left[(x, y)_{\omega_{d}}\right] } & \longmapsto\left[\left(\mathrm{N}_{K / F}(x), \mathrm{N}_{K / F}(y)\right)_{\omega_{d s}}\right] .
\end{aligned}
$$

Proof. The explicit descriptions of first two maps are well known. They follow immediately from the remarks preceding Theorem 2.8, which describe the inflation and restriction on alternating forms, and then apply Theorem 4.4 and Lemma 4.2 to the case $\Omega=F_{\mathrm{ab}}$. Since inflation and restriction are morphisms of cohomological functors, the induced maps on $\mathrm{Br}^{\circ}(K / F)$ are indeed inflation and restriction. The latter is injective on Brauer groups. By Theorem 2.8, $\iota^{* \wedge 2}: \operatorname{Alt}\left(\mathrm{G}_{F_{\mathrm{ab}} / F}, \mathbb{Q} / \mathbb{Z}\right) \rightarrow$ $\operatorname{Alt}\left(\mathrm{G}_{F_{\mathrm{ab}} / K}, \mathbb{Q} / \mathbb{Z}\right)$ is surjective, since $\mathrm{G}_{F_{\mathrm{ab}} / F}$ is torsion-free. Therefore the restriction $\operatorname{Br}^{\circ}\left(F_{\mathrm{ab}} / F\right)=\operatorname{Br}(F) \rightarrow \operatorname{Br}^{\circ}\left(F_{\mathrm{ab}} / K\right)$ is surjective.

Suppose $x, y \in F_{\mathrm{ab}}^{d} \cap K^{\times}$, so $\left[(x, y)_{\omega_{d}}\right] \in \mathrm{Br}^{\circ}\left(F_{\mathrm{ab}} / K\right)$ is a general element, by Theorem 4.4. By Theorem 2.10, the corestriction on $\operatorname{Alt}\left(\mathrm{G}_{F_{\mathrm{ab}} / K}, \mathbb{Q} / \mathbb{Z}\right)$ is obtained by applying $\jmath^{* \wedge 2}$ to a choice of $s$-th root. Since the transfer $\jmath$ induces a welldefined map on Brauer groups, its effect on symbols can be computed directly using Theorem 4.4. By definition, the corestriction on alternating forms corresponds to corestriction on cohomology with coefficients in $\mathbb{Q} / \mathbb{Z}$, under the natural map. Since cor is a morphism of cohomological functors, the map $\varepsilon_{*} \cdot \omega_{*}: \mathbb{Q} / \mathbb{Z} \rightarrow F_{\mathrm{ab}}^{\times}$induces a commutative diagram

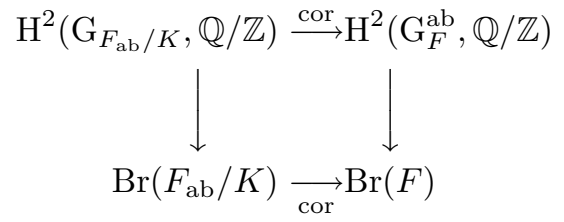

Therefore, to compute the corestriction on $\mathrm{Br}^{\circ}\left(F_{\mathrm{ab}} / K\right)$, it suffices to find a preimage of $\left[(x, y)_{\omega_{d}}\right] \in \operatorname{Br}\left(F_{\mathrm{ab}} / K\right)$ in $\operatorname{Alt}\left(\mathrm{G}_{F_{\mathrm{ab}} / K}, \mathbb{Q} / \mathbb{Z}\right)$, take an $s$-th root, apply $\kappa^{\wedge 2}$, and use Lemma 4.2 to apply $\jmath^{*}$ to each parameter of the resulting symbol, as in Theorem 4.4.

Let $\phi^{*}, \psi^{*} \in{ }_{d} \mathrm{G}_{F_{\mathrm{ab}} / K}^{*}$ be preimages of $x, y \in\left(\Omega^{d} \cap K^{\times}\right) / K^{\times d}$ under the Kummer isomorphism $\kappa_{\omega_{d}}$. By Theorem 4.4, $\kappa^{\wedge 2}\left(\phi^{*} \wedge_{d} \psi^{*}\right)=\left[(x, y)_{\omega_{d}}\right]$. An easy computation (again) using (2.3.1) shows $\frac{1}{s} \phi^{*} \wedge_{d s} \frac{1}{s} \psi^{*}$ is an $s$-th root of $\phi^{*} \wedge_{d} \psi^{*}$. By 
(4.1.1), the multiplication by $s$ map ${ }_{d s} \mathrm{G}_{F_{\mathrm{ab}} / K}^{*} \rightarrow{ }_{d} \mathrm{G}_{F_{\mathrm{ab}} / K}$ induces the natural map $\left(F_{\mathrm{ab}}^{d s} \cap K^{\times}\right) / K^{\times} d s \rightarrow\left(F_{\mathrm{ab}}^{d} \cap K^{\times}\right) / K^{\times d}$. Therefore let $x$ and $y$ also denote the images $\kappa_{\omega_{d s}}\left(\frac{1}{s} \phi^{*}\right)$ and $\kappa_{\omega_{d s}}\left(\frac{1}{s} \psi^{*}\right)$, respectively. By Theorem 4.4,

$$
\kappa^{\wedge 2}\left(\frac{1}{s} \phi^{*} \wedge_{d s} \frac{1}{s} \psi^{*}\right)=\left[(x, y)_{\omega_{d s}}\right] .
$$

By functoriality, $\jmath^{* \wedge 2}\left(\frac{1}{s} \phi^{*} \wedge_{d s} \frac{1}{s} \psi^{*}\right)=\jmath^{*}\left(\frac{1}{s} \phi^{*}\right) \wedge_{d s} \jmath^{*}\left(\frac{1}{s} \psi^{*}\right)$. Therefore by definition of the Kummer transpose,

$$
\kappa^{\wedge 2}\left(\jmath^{* \wedge 2}\left(\frac{1}{s} \phi^{*} \wedge_{d s} \frac{1}{s} \psi^{*}\right)\right)=\left[\left(\jmath^{*}(x), \jmath^{*}(y)\right)_{\omega_{d s}}\right] .
$$

Since by Lemma $4.2, \jmath^{*}(x)=\mathrm{N}_{K / F}(x)$ and $\jmath^{*}(y)=\mathrm{N}_{K / F}(y)$, this completes the proof.

Remarks 4.13. a. In the situation of Theorem 4.12, suppose $y \in F^{\times}$and $x \in$ $F_{\mathrm{ab}}^{d} \cap K^{\times}$. Then $\left[(x, y)_{\omega_{d}}\right] \in \operatorname{Br}^{\circ}\left(F_{\mathrm{ab}} / K\right)$, and

$$
\operatorname{cor}\left(\left[(x, y)_{\omega_{d}}\right]\right)=\left[\left(\mathrm{N}_{K / F}(x), y^{s}\right)_{\omega_{d s}}\right]=s\left[\left(\mathrm{~N}_{K / F}(x), y\right)_{\omega_{d s}}\right]=\left[\left(\mathrm{N}_{K / F}(x), y\right)_{\omega_{d}}\right],
$$

which agrees with the well-known theorem [D, Section 11, Theorem 7] (which applies whether or not $K / F$ is abelian).

b. If $\mathrm{G}_{F}$ is abelian, then $F_{\mathrm{ab}}=K_{\mathrm{ab}}$, hence $\mathrm{Br}^{\circ}\left(F_{\mathrm{ab}} / K\right)=\mathrm{Br}(K)$. This situation occurs, for example, when $F=\mathbb{C}\left(\left(x_{1}\right)\right) \cdots\left(\left(x_{n}\right)\right)$, the field of iterated power series. Then the restriction is surjective, and the corestriction formula applies to all of $\operatorname{Br}(K)$.

\section{BASES}

Let $G$ be a proabelian group. One of the main goals of this paper is to effectively compute the theory of the $\operatorname{group} \operatorname{Alt}(G, \mathbb{Q} / \mathbb{Z})$ of continuous alternating forms on $G$, and then, when $G$ is the Galois group over a field $F$ containing $\mu$, to effectively compute the homomorphism $\operatorname{Alt}(G, \mathbb{Q} / \mathbb{Z}) \rightarrow \operatorname{Br}(F)$ resulting from the natural isomorphism $\operatorname{Alt}(G, \mathbb{Q} / \mathbb{Z}) \simeq \mathrm{H}^{2}(G, \mathbb{Q} / \mathbb{Z})$.

The next two sections show how to use bases to achieve this goal when $G$ has homogeneous primary components, in particular when $G$ is torsion-free or torsion. Bases are first defined, then dualized, and then extended to the groups $\operatorname{Bil}(G, \mathbb{Q} / \mathbb{Z})$ and $\operatorname{Alt}(G, \mathbb{Q} / \mathbb{Z})$. Compatible bases are then defined in connection with the ground field $F$, where they produce a set of generators for $\operatorname{Br}(F)$.

All of this would be somewhat simplified if it could be assumed that $G$ was a finite product of procyclic groups of some fixed type, like $\mathbb{Z}_{p}, \widehat{\mathbb{Z}}$, or $\mathbb{Z} / d \mathbb{Z}$. However, in order for the map to $\operatorname{Br}(F)$ to be surjective, it must be assumed that $G$ is the Galois group of the maximal abelian extension of $F$, a generally inhomogeneous, generally infinite product of various $\mathbb{Z}_{p}$ 's. For this reason, a possibly inhomogeneous proabelian $G$ will be treated. It will be assumed, however, that in addition to having homogeneous primary components, $G$ is either all torsion or all torsion-free.

\section{Bases for $G$ and $G^{*}$}

Suppose $G$ is a proabelian group that is either torsion or torsion-free. By the structure theory ([RZ, Theorem 4.3.3, Theorem 4.3.9]), there is a topological isomorphism $G \simeq \prod_{I} \mathrm{G}_{i}$, where $I$ is a set, $\mathrm{G}_{i}$ is procyclic, and $\prod_{I} \mathrm{G}_{i}$ has the product topology. In the torsion-free case, $\mathrm{G}_{i}=\mathbb{Z}_{\mathfrak{m}_{i}}$, for some $\mathfrak{m}_{i} \subset \mathcal{P}$, and in the torsion case $\mathrm{G}_{i}=\mathbb{Z} / d_{i} \mathbb{Z}$, for some $d_{i} \in \mathbb{N}$ that divides the exponent. By [RZ, Lemma 4.3.7], a torsion proabelian group has bounded exponent. If $\mathrm{G}_{i} \simeq \mathbb{Z}_{\mathfrak{m}_{i}}, \phi \in \mathrm{G}_{i}$, and 
$c \in \mathbb{Z}_{\mathfrak{m}_{i}}$, then $\phi^{c}$ is the element whose image in each finite quotient $\mathrm{G}_{i} / \mathrm{G}_{i}^{d}$ is $\phi_{d}^{c_{d}}$, where $\phi_{d}$ and $c_{d}$ are elements of $\mathrm{G}_{i} / \mathrm{G}_{i}^{d}$ and $\mathbb{Z}_{\mathfrak{m}_{i}} / d \mathbb{Z}_{\mathfrak{m}_{i}}$, respectively.

By Pontrjagin Duality [RZ, Lemma 2.9.4], $G \simeq \prod_{I} \mathrm{G}_{i}$ if and only if $G^{*} \simeq \coprod_{I} \mathrm{G}_{i}^{*}$, where in the torsion-free case $\mathrm{G}_{i}=\mathbb{Z}_{\mathfrak{m}_{i}}$ if and only if $\mathrm{G}_{i}^{*}=\mathbb{Z}\left[1 / \mathfrak{m}_{i}\right] / \mathbb{Z}$, and in the torsion-case, $\mathrm{G}_{i}=\mathbb{Z} / d_{i} \mathbb{Z}$ if and only if $\mathrm{G}_{i}^{*}=\left(1 / d_{i}\right) \mathbb{Z} / \mathbb{Z}$. Thus a proabelian group is torsion-free if and only if its dual is divisible, and it is torsion if and only if its dual has bounded exponent.

The guiding example for the definition below arises when $G$ is isomorphic to a finite homogeneous product of torsion-free procyclic groups, all of the same type, such as $\widehat{\mathbb{Z}}^{n}$. By a basis for such a group is meant a topological basis, given by the images of the canonical generators under a topological isomorphism $\widehat{\mathbb{Z}}^{n} \stackrel{\sim}{\longrightarrow} G$. It is then easy to define $d u a l$ bases for the $d$-torsion subgroups ${ }_{d} G^{*}$, using the transpose ${ }_{d} G^{*} \stackrel{\sim}{\longrightarrow}\left(\frac{1}{d} \mathbb{Z} / \mathbb{Z}\right)^{n}$. In fact, for practical purposes this is the only case that will be needed. For if $\boldsymbol{a}$ is any alternating form on an arbitrary torsion-free proabelian $G$, then by continuity $\boldsymbol{a}$ 's radical contains all but finitely many factors in any product representation of $G$. Moreover, $\boldsymbol{a}$ 's $p$-primary components are all supported on the $p$-primary part of $G$, which is a product of $\mathbb{Z}_{p}$ 's. Thus the computation of matrix forms for any particular $\boldsymbol{a}$ can always be reduced to the case where $G$ is a finite homogeneous product of procyclic groups of the same type. Here is the general definition.

Definitions 5.1. a. Let $G$ be a torsion or torsion-free proabelian group. A topological basis for $G$ is the image of the standard generators $\left\{\mathbf{e}_{i}\right\}$ under a topological isomorphism

$$
\Phi: \prod_{I} \mathrm{G}_{i} \stackrel{\sim}{\longrightarrow} G
$$

where each $\mathrm{G}_{i}$ is procyclic, with canonical generator $\mathbf{e}_{i}$. Let $\phi_{i}=\Phi\left(\mathbf{e}_{i}\right)$, and write $\underline{\phi}=\left\{\phi_{i}\right\}_{I}$. Partition $I$ into a disjoint union $\bigcup_{J} I_{j}$, defined for each $j \in J$ by $\bar{I}_{j}=\left\{i \in I: \mathrm{G}_{i} \simeq \mathrm{G}_{j}\right\}$. Then

$$
\prod_{I} \mathrm{G}_{i}=\prod_{J} \prod_{I_{j}} \mathrm{G}_{i}=\prod_{J} \mathrm{G}_{j}^{\left|I_{j}\right|} .
$$

Call $\mathrm{G}_{j}^{\left|I_{j}\right|}$ a homogeneous component. Call a decomposition $\Phi$ and the corresponding basis $\underline{\phi}$ homogeneous if the $\mathrm{G}_{i}$ are all isomorphic. Call $\Phi$ and $\underline{\phi}$ primary if the $\mathrm{G}_{i}$ are all $p$-groups, for various $p$.

A topological basis change is given by a commutative diagram

$$
\begin{aligned}
G & =G \\
\Phi^{\prime} \uparrow & \uparrow \Phi \\
\prod_{I} \mathrm{G}_{i} & \longrightarrow \prod_{I} \mathrm{G}_{i}
\end{aligned}
$$

where $\Phi, \Phi^{\prime}$, and $\iota$ are topological isomorphisms, and $\iota$ 's restriction to each homogeneous component $\mathrm{G}_{j}^{\left|I_{j}\right|}$ is an isomorphism onto $\mathrm{G}_{j}^{\left|I_{j}\right|}$. A basis change on $G$ is given explicitly by $\phi_{j}^{\prime}=\prod_{i} \phi_{i}^{p_{i j}}$, where in the torsion-free case, $p_{i j} \in \mathbb{Z}_{\mathfrak{m}_{i}}$, and in the torsion case, $p_{i j} \in \mathbb{Z} / d_{i} \mathbb{Z}$, where $d_{i}=\left|\mathrm{G}_{i}\right|$. If $\theta \in G$, let $[\theta]_{\underline{\phi}}$ denote $\theta$ 's coordinate 
expression with respect to $\underline{\phi}$, and let $\mathcal{P}=[\iota]^{\frac{\phi}{\phi^{\prime}}}$ be $\iota$ 's coordinate expression with respect to $\underline{\phi}^{\prime}$ and $\underline{\phi}$. Then write $\mathcal{P}:[\theta]_{\underline{\phi}^{\prime}} \mapsto[\theta]_{\underline{\phi}}$ on $G$, and

$$
\underline{\phi}^{\prime}=\underline{\phi} \mathcal{P} \text {. }
$$

b. Let $G^{*}$ be a discrete torsion abelian group of bounded exponent dividing $d$. A basis for $G^{*}$ is the preimage of the set of canonical generators $\mathbf{e}_{i}^{*} \in \mathrm{G}_{i}^{*}=\frac{1}{d_{i}} \mathbb{Z} / \mathbb{Z}$, $d_{i} \mid d$, under an isomorphism

$$
\Phi^{*}: G^{*} \stackrel{\sim}{\longrightarrow} \coprod_{I} \mathrm{G}_{i}^{*} .
$$

Let $\phi_{i}^{*}=\left(\Phi^{*}\right)^{-1}\left(\mathbf{e}_{i}^{*}\right)$, and write $\phi^{*}=\left\{\phi_{i}^{*}\right\}$. Decompose $I$ into a disjoint union $I=\bigcup_{J} I_{j}$ as in (a), with $I_{j}=\left\{i \in I: \mathrm{G}_{i}^{*} \simeq \mathrm{G}_{j}^{*}\right\}$. Then $\coprod_{I} \mathrm{G}_{i}^{*}=\coprod_{J}\left(\coprod_{I_{j}} \mathrm{G}_{i}^{*}\right)$. Say $G^{*}$ is homogeneous if the $\mathrm{G}_{i}^{*}$ are isomorphic for all $i$. A basis change from $\underline{\phi}^{* \prime}$ to $\underline{\phi}^{*}$ is given by a commutative diagram

$$
\begin{gathered}
G^{*}=G^{*} \\
\Phi^{\prime *} \downarrow \\
\coprod_{I} \mathrm{G}_{i}^{*} \longleftarrow \coprod_{\iota^{*}} \coprod_{I} \mathrm{G}_{i}^{*}
\end{gathered}
$$

where $\Phi^{*}, \Phi^{* *}$, and $\iota^{*}$ are isomorphisms, and $\iota^{*}$ maps each $\coprod_{I_{j}} \mathrm{G}_{i}^{*}$ onto $\coprod_{I_{j}} \mathrm{G}_{i}^{*}$.

A basis change is given explicitly by $\phi_{j}^{*}=\sum_{i} p_{i j}^{*} \phi_{i}^{* \prime}$ for elements $p_{i j}^{*} \in \mathbb{Z} / d_{i} \mathbb{Z}$, finitely many of which are nonzero. If $d_{i} \in \mathbb{N}\left(\mathfrak{m}_{i}\right)$, then $\mathbb{Z} / d_{i} \mathbb{Z}$ is a quotient of $\mathbb{Z}_{\mathfrak{m}_{i}}$, and the $p_{i j}^{*}$ may be represented in $\mathbb{Z}_{\mathfrak{m}_{i}}$. Let $\mathcal{P}^{*}=\left[\iota^{*}\right] \frac{\phi^{* \prime}}{\phi^{*}}$ be $\iota^{*}$ 's coordinate expression with respect to $\underline{\phi}^{*}$ and $\underline{\phi}^{* \prime}$. Then for $\theta^{*} \in G^{*}, \mathcal{P}^{*}:\left[\theta^{*}{\underline{\phi^{*}}} \mapsto\left[\theta^{*}\right]_{\underline{\phi}^{* \prime}}\right.$, and

$$
\underline{\phi}^{* \prime} \mathcal{P}^{*}=\underline{\phi}^{*} .
$$

c. Let $G$ be a torsion or torsion-free proabelian group. Suppose $d \in \mathbb{N}$ divides

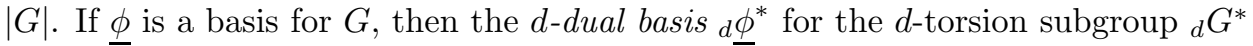
is defined by

$$
{ }_{d} \phi_{i}^{*}\left(\phi_{j}\right)=\delta_{i j} / d_{i} \in \mathbb{Q} / \mathbb{Z}
$$

where $d_{i}=\left(d,\left|\phi_{i}\right|\right)=\left|{ }_{d} \phi_{i}^{*}\right|$. A basis change ${ }_{d} \phi^{*} \mathcal{P}^{*}={ }_{d} \phi^{*}$ on ${ }_{d} G^{*}$ is the transpose of a topological basis change $\underline{\phi}^{\prime}=\phi \mathcal{P}$ on $G$ if ${ }_{d} \underline{\phi}^{* *}$ and ${ }_{d} \underline{\phi}^{*}$ are dual to $\underline{\phi}^{\prime}$ and $\underline{\phi}$, respectively. Then ${ }_{d} \phi_{j}^{*}=\sum_{i} \bar{p}_{i j}^{*}\left({ }_{d} \phi_{i}^{\prime *}\right)$ and $p_{i j}^{*} \equiv \bar{p}_{j i}\left(\bmod \bar{d}_{i}\right)$. For compute

$$
p_{j i} / d_{i}={ }_{d} \phi_{j}^{*}\left(\prod_{k \in I} \phi_{k}^{p_{k i}}\right)=\left(\sum_{k \in I} p_{k j}^{*}\left({ }_{d} \phi_{k}^{\prime *}\right)\right)\left(\phi_{i}^{\prime}\right)=p_{i j}^{*} / d_{i} .
$$

Since this holds for all $d$ dividing $|G|$, in the torsion-free case the coefficients $p_{i j}^{*}$ can be taken to lie in $\mathbb{Z}_{\mathfrak{m}_{i}}$, and then $p_{i j}^{*}=p_{j i} \in \mathbb{Z}_{\mathfrak{m}_{i}}$. In this case, write

$$
\underline{\phi}^{* *} \mathcal{P}^{*}=\underline{\phi}^{*}
$$

as shorthand for the family of basis changes $\left\{{ }_{d} \underline{\phi}^{* *} \mathcal{P}^{*}={ }_{d} \underline{\phi}^{*}\right\}$.

Example 5.2. If $G=\widehat{\mathbb{Z}}^{n}$, the free proabelian group of rank $n$, then the standard basis element is $\phi=\left\{1_{i}\right\}_{i=1}^{n}$, where $1_{i}$ is the $i$-th summand's canonical generator. A basis change is represented by a matrix $\mathcal{P}=\left(p_{i j}\right) \in \mathrm{GL}_{n}(\widehat{\mathbb{Z}})$. The new basis $\underline{\phi}^{\prime}=\underline{\phi} \mathcal{P}$ is given by $\phi_{j}^{\prime}=\prod_{i=1}^{n} \phi_{i}^{p_{i j}}$. The dual of $G$ is $G^{*}=(\mathbb{Q} / \mathbb{Z})^{n}$. The basis for ${ }_{d} G^{*}$ dual to $\underline{\phi}$ is ${ }_{d} \underline{\phi}^{*}=\left\{(1 / d)_{i}\right\}_{i=1}^{n}$, where $(1 / d)_{i}$ is the $i$-th summand's canonical 
generator. Set $\underline{\phi}^{*}=\left\{{ }_{d} \underline{\phi}^{*}\right\}$. If $\mathcal{P} \in \mathrm{GL}_{n}(\widehat{\mathbb{Z}})$ is a basis change for $G, \mathcal{P}^{*} \in \mathrm{GL}_{n}(\widehat{\mathbb{Z}})$ is the matrix transpose of $\mathcal{P}$, and the transpose basis change is $\underline{\phi}^{*} \mathcal{P}^{*}=\underline{\phi}^{*}$, so ${ }_{d} \phi_{j}^{*}=\sum_{i=1}^{n} p_{i j d}^{*} \phi_{i}^{* \prime}$.

By the above definitions, a given topological basis $\phi$ for a torsion-free proabelian group $G$ determines a system $\phi^{*}=\left\{{ }_{d} \phi^{*}\right\}$ of dual bases for the various torsion groups ${ }_{d} G^{*}$. The dual bases for each homogeneous component are coherent in the following sense.

Lemma 5.3. Suppose $G=\prod_{I} \mathrm{G}_{i}$ is homogeneous, with each $\mathrm{G}_{i} \simeq \mathbb{Z}_{\mathfrak{m}}$, for some $\mathfrak{m} \subset \mathcal{P}$. Let $\phi$ be a homogeneous topological basis. Then for all $d, e \in \mathbb{N}$ such that $d \mid e$ and $e / d \in \mathbb{N}(\mathfrak{m}),{ }_{d} \underline{\phi}^{*}=(e / d)_{e} \phi^{*}$. That is, ${ }_{d} \phi_{i}^{*}=(e / d)_{e} \phi_{i}^{*}$ for all $i \in I$. Conversely, suppose $G^{*} \equiv \coprod_{I} \mathrm{G}_{i}^{*}$ is homogeneous, with each $\mathrm{G}_{i}^{*} \simeq \mathbb{Z}[1 / \mathfrak{m}] / \mathbb{Z}$. Let $\underline{\phi}^{*}=\left\{{ }_{d} \underline{\phi}^{*}: d \in \mathbb{N}(\mathfrak{m})\right\}$ be a family of d-bases for $G^{*}$ such that ${ }_{d} \underline{\phi}^{*}=(e / d)_{e} \underline{\phi}^{*}$ $\overline{\text { whenever }} d \mid e$ and $e \in \mathbb{N}(\mathfrak{m})$. Then there exists a topological basis $\underline{\phi}$ for $G=\prod_{I} \overline{\mathrm{G}}_{i}$ that is dual to $\left\{{ }_{d} \underline{\phi}^{*}\right\}$, meaning each ${ }_{d} \underline{\phi}^{*}$ is dual to $\underline{\phi}$.

Proof. For the first statement, let $e_{i}=\left(e,\left|\phi_{i}\right|\right)$ and $d_{i}=\left(d,\left|\phi_{i}\right|\right)$; then $e / d=e_{i} / d_{i}$ since $e / d \in \mathbb{N}(\mathfrak{m})$, so ${ }_{d} \phi_{i}^{*}\left(\phi_{j}\right)=\delta_{i j} / d_{i}=(e / d)\left(\delta_{i j} / e_{i}\right)=(e / d)_{e} \phi_{i}^{*}\left(\phi_{j}\right)$.

For the converse, let $G$ be the (proabelian) dual of $G^{*}$; then $G=\prod_{I} \mathrm{G}_{i}$, where $\mathrm{G}_{i}$ is the procyclic dual of $\mathrm{G}_{i}^{*}$. Let ${ }_{d} \underline{\phi}$ be the dual of ${ }_{d} \underline{\phi}^{*}$ in $G / G^{d}$, for all $d$. If $d \mid e$ and $e \in \mathbb{N}(\mathfrak{m})$, the dual of ${ }_{e} \underline{\phi}$ in ${ }_{d} G^{*}$ is ${ }_{d} \underline{\phi}^{*}$, since

$$
{ }_{d} \phi_{i}^{*}\left({ }_{e} \phi_{j}\right)=\left((e / d)_{e} \phi_{i}^{*}\right)\left({ }_{e} \phi_{j}\right)=(e / d)\left(\delta_{i j} / e_{i}\right)=\delta_{i j} / d_{i} .
$$

Therefore the image of $e_{e} \underline{\phi}$ in $G / G^{d}$ is ${ }_{d} \underline{\phi}$, and $\left\{{ }_{d} \underline{\phi}: d \in \mathbb{N}(\mathfrak{m})\right\}$ is a projective system of sets. Since $G$ is $\mathfrak{m}$-homogeneous, $G \simeq \lim _{d} \bar{G} / G^{d}$, where the limit is taken over $d \in \mathbb{N}(\mathfrak{m})$. Let

$$
\underline{\phi}=\lim _{d \in \mathbb{N}(\mathfrak{m})}(d \underline{\phi}) \subset G,
$$

so that $\phi_{i}=\lim _{d}\left({ }_{d} \phi_{i}\right)$. Each $\phi_{i} \in \underline{\phi}$ generates $\mathrm{G}_{i}$ topologically, so it is clear that $\underline{\phi}$ is a topological basis for $G$. Moreover, ${ }_{d} \phi_{i}^{*}\left(\phi_{j}\right)=\delta_{i j} / d_{i}$, so $\underline{\phi}$ is dual to $d \underline{\phi}^{*}$.

Bases for $\operatorname{Bil}(G, \mathbb{Q} / \mathbb{Z}), \mathrm{H}^{2}(G, \mathbb{Q} / \mathbb{Z})$, and $\operatorname{Alt}(G, \mathbb{Q} / \mathbb{Z})$

Suppose $G$ is a proabelian group that is either torsion-free or torsion. A (topological) basis $\underline{\phi}=\left\{\phi_{i}\right\}_{I}$ for $G$ determines compatible dual $d$-bases $\left\{{ }_{d} \phi_{i}^{*}\right\}$. Let $d_{i}=\left|{ }_{d} \phi_{i}^{*}\right|$, and let $d_{i j}=\operatorname{gcd}\left(d_{i}, d_{j}\right)$. Consider the elements $\left({ }_{d} \phi_{i}^{*}\right) \otimes_{\left(d_{i}, d_{j}\right)}\left({ }_{d} \phi_{j}^{*}\right)$ and $\left({ }_{d} \phi_{i}^{*}\right) \wedge_{\left(d_{i}, d_{j}\right)}\left({ }_{d} \phi_{j}^{*}\right)$, in $\operatorname{Bil}(G, \mathbb{Q} / \mathbb{Z})$ and $\operatorname{Alt}(G, \mathbb{Q} / \mathbb{Z})$, respectively. Their action is described explicitly by

$$
\left({ }_{d} \phi_{i}^{*}\right) \otimes_{\left(d_{i}, d_{j}\right)}\left({ }_{d} \phi_{j}^{*}\right)\left(\prod_{k \in I} \phi_{k}^{a_{k}}, \prod_{k \in I} \phi_{k}^{b_{k}}\right)=\frac{a_{i} b_{j}}{d_{i j}} \quad \in \mathbb{Q} / \mathbb{Z}
$$

and

$$
\left({ }_{d} \phi_{i}^{*}\right) \wedge_{\left(d_{i}, d_{j}\right)}\left({ }_{d} \phi_{j}^{*}\right)\left(\prod_{k \in I} \phi_{k}^{a_{k}}, \prod_{k \in I} \phi_{k}^{b_{k}}\right)=\frac{a_{i} b_{j}-a_{j} b_{i}}{d_{i j}} \in \mathbb{Q} / \mathbb{Z} .
$$

To control notation, set

$$
\begin{aligned}
\left(\phi_{i}^{*} \otimes \phi_{j}^{*}\right)_{d} & \left.:={ }_{d} \phi_{i}^{*}\right) \otimes_{\left(d_{i}, d_{j}\right)}\left({ }_{d} \phi_{j}^{*}\right), \\
\left(\phi_{i}^{*} \wedge \phi_{j}^{*}\right)_{d} & \left.:={ }_{d} \phi_{i}^{*}\right) \wedge_{\left(d_{i}, d_{j}\right)}\left({ }_{d} \phi_{j}^{*}\right) .
\end{aligned}
$$

Thus $\left|\left(\phi_{i}^{*} \wedge \phi_{j}^{*}\right)_{d}\right|=d_{i j}$. 
Proposition 5.4. Suppose $G$ is a torsion-free proabelian group, with topological basis $\underline{\phi}=\left\{\phi_{i}\right\}_{i \in I}$, indexed by a linearly ordered set I. Then

$$
\begin{aligned}
{ }_{d} \underline{\phi}^{* \otimes 2} & :=\left\{\left(\phi_{i}^{*} \otimes \phi_{j}^{*}\right)_{d}: i, j \in I \text { and }\left(\phi_{i}^{*} \otimes \phi_{j}^{*}\right)_{d} \neq 0\right\}, \\
{\left[{ }_{d} \underline{\phi}^{* \otimes 2}\right]: } & :=\left\{\left[\left(\phi_{i}^{*} \otimes \phi_{j}^{*}\right)_{d}\right]: i<j \in I \text { and }\left[\left(\phi_{i}^{*} \otimes \phi_{j}^{*}\right)_{d}\right] \neq 0\right\}, \\
{ }_{d} \underline{\phi}^{* \wedge 2}: & =\left\{\left(\phi_{i}^{*} \wedge \phi_{j}^{*}\right)_{d}: i<j \in I \text { and }\left(\phi_{i}^{*} \wedge \phi_{j}^{*}\right)_{d} \neq 0\right\}
\end{aligned}
$$

are bases for ${ }_{d} \operatorname{Bil}(G, \mathbb{Q} / \mathbb{Z}),{ }_{d} \mathrm{H}^{2}(G, \mathbb{Q} / \mathbb{Z})$ and ${ }_{d} \operatorname{Alt}(G, \mathbb{Q} / \mathbb{Z})$, respectively.

Proof. Since $G$ is torsion-free, $\otimes_{d}^{2} G^{*}$ generates ${ }_{d} \operatorname{Bil}(G, \mathbb{Q} / \mathbb{Z})$ by Theorem 2.4. By the bilinearity of the tensor product, $\otimes_{d}^{2} G^{*}$ is spanned by the elements $\left({ }_{d} \phi_{i}^{*}\right) \otimes_{d}$ $\left({ }_{d} \phi_{j}^{*}\right)$. Let $d_{i}=\left|{ }_{d} \phi_{i}^{*}\right|$, and let $d_{i j}=\operatorname{gcd}\left(d_{i}, d_{j}\right)$ for each $i, j \in I$. Let $u=$ $d / \operatorname{lcm}\left[d_{i}, d_{j}\right]$. Since $G$ has homogeneous primary components, $\operatorname{gcd}\left(u, \operatorname{lcm}\left[d_{i}, d_{j}\right]\right)=$ 1 , hence $u$ is a $d_{i j}$-unit. By $(2.3 .2), u \cdot \nu_{d_{i}, d_{j}}=\nu_{d}$, and it follows that

$$
\left\langle\left({ }_{d} \phi_{i}^{*}\right) \otimes_{d}\left({ }_{d} \phi_{j}^{*}\right)\right\rangle=\left\langle\left(\phi_{i}^{*} \otimes_{d} \phi_{j}^{*}\right)_{d}\right\rangle \text {. }
$$

Thus $\otimes^{2}{ }_{d} G^{*}$ is spanned by ${ }_{d} \underline{\phi}^{* \otimes 2}$. Since $\left(\phi_{i}^{*} \wedge \phi_{j}^{*}\right)_{d}=-\left(\phi_{j}^{*} \wedge \phi_{i}^{*}\right)_{d},{ }_{d} \underline{\phi}^{* \wedge 2}$ generates ${ }_{d} \operatorname{Alt}(G, \mathbb{Q} / \mathbb{Z})$. It follows that $\left[{ }_{d} \phi^{* \otimes 2}\right]$ generates ${ }_{d} \mathrm{H}^{2}(G, \mathbb{Q} / \mathbb{Z})$ by Theorem 2.4. In fact, check directly that $\left(\phi_{i}^{*} \otimes \phi_{j}^{*}\right)_{d}+\left(\phi_{j}^{*} \otimes \phi_{i}^{*}\right)_{d}$ equals the coboundary $\partial h$ of the 1cochain $h \in C^{1}(G, \mathbb{Q} / \mathbb{Z})$ given by $h\left(\prod_{k} \phi_{k}^{a_{k}}\right)=-a_{i} a_{j} / d_{i j}$. Therefore $\left[\left(\phi_{i}^{*} \otimes \phi_{j}^{*}\right)_{d}\right]=$ $-\left[\left(\phi_{j}^{*} \otimes \phi_{i}^{*}\right)_{d}\right]$ in $\mathrm{H}^{2}(G, \mathbb{Q} / \mathbb{Z})$.

If $\boldsymbol{a}=\sum n_{i j}\left(\phi_{i}^{*} \otimes \phi_{j}^{*}\right)_{d}=0$, then $\boldsymbol{a}\left(\phi_{i}, \phi_{j}\right)=n_{i j} / d_{i j}=0$, where for all $i, j \in I$, $d_{i j}=\operatorname{gcd}\left(d,\left|\phi_{i}\right|,\left|\phi_{j}\right|\right)$. Therefore ${ }_{d} \phi^{* \otimes 2}$ is an independent set, hence a basis for ${ }_{d} \operatorname{Bil}(G, \mathbb{Q} / \mathbb{Z})$. Similarly, ${ }_{d} \underline{\phi}^{* \wedge 2}$ is a basis for ${ }_{d} \operatorname{Alt}(G, \mathbb{Q} / \mathbb{Z})$, and since the isomorphism of Theorem 2.4 sends ${ }_{d} \underline{\phi}^{* \wedge 2}$ to $\left[{ }_{d} \underline{\phi}^{* \otimes 2}\right],\left[{ }_{d} \underline{\phi}^{* \otimes 2}\right]$ is a basis for ${ }_{d} \mathrm{H}^{2}(G, \mathbb{Q} / \mathbb{Z})$.

Definition 5.5. Suppose $\underline{\phi}=\left\{\phi_{i}\right\}_{I}$ is a basis for $G$, indexed by a linearly ordered set $I, \boldsymbol{b} \in \operatorname{Bil}(G, \mathbb{Q} / \mathbb{Z})$, and $\boldsymbol{a} \in \operatorname{Alt}(G, \mathbb{Q} / \mathbb{Z})$. Set $b_{i j}=\boldsymbol{b}\left(\phi_{i}, \phi_{j}\right)$ and $a_{i j}=$ $\boldsymbol{a}\left(\phi_{i}, \phi_{j}\right)$. The coordinate (or matrix) expressions $[\boldsymbol{b}]_{\underline{\phi}^{* \otimes 2}}$ and $[\boldsymbol{a}]_{\underline{\phi}^{* \wedge 2}}$, or just $[\boldsymbol{b}]_{\underline{\phi}}$ and $[\boldsymbol{a}]_{\underline{\underline{\phi}}}$ if the meaning is clear, are the $I \times I$ matrices $\left(b_{i j}\right)$ and $\left(a_{i j}\right)$. Note that almost all of the $b_{i j}$ and $a_{i j}$ are zero, and if $b_{i j}=n_{i j} / d_{i j}$ and $a_{i j}=m_{i j} / d_{i j}$ in $\mathbb{Q} / \mathbb{Z}$, then $\boldsymbol{b}=\sum_{i, j} n_{i j}\left(\phi_{i}^{*} \otimes \phi_{j}^{*}\right)_{d}$ and $\boldsymbol{a}=\sum_{i<j} m_{i j}\left(\phi_{i}^{*} \wedge \phi_{j}^{*}\right)_{d}$, where $d_{i j}=$ $\operatorname{gcd}\left(d,\left|\phi_{i}\right|,\left|\phi_{j}\right|\right)$. Let

$$
\mathrm{M}_{|\underline{\phi}|}(\mathbb{Q} / \mathbb{Z}), \quad \operatorname{Alt}_{|\underline{\phi}|}(\mathbb{Q} / \mathbb{Z})
$$

denote the group of all such matrices, with pointwise addition.

Remark 5.6. The coordinate (matrix) expression is a matrix representative, in the sense of Definition 3.2. Moreover, every matrix representative is a matrix expression, for some basis. For suppose $\left(b_{i j}\right)$ is the matrix for $\boldsymbol{b}$, determined by a basis $\bar{\phi}$ for $G / \operatorname{rad}_{b}(G)$. It will be shown in Lemma 5.11, below, that $\underline{\phi}$ extends to a basis $\underline{\phi}$ for $G$, hence $\left(b_{i j}\right)=[\boldsymbol{b}]_{\underline{\phi}}$.

Proposition 5.7 "Basis Change on Bilinear and Alternating Forms". Suppose $G$ is a torsion-free proabelian group with topological basis $\phi$. Suppose $\phi^{\prime}=\phi \mathcal{P}$ is a topological basis change on $G$, defined explicitly by $\phi_{j}^{\prime}=\bar{\prod}_{I} \phi_{i}^{p_{i j}}, p_{i j} \in \mathbb{Z}_{\mathfrak{m}_{i}}^{-}$. Then the transpose basis changes

$$
\left({ }_{d} \underline{\phi}^{* \otimes 2}\right) \mathcal{P}^{* \otimes 2}={ }_{d} \underline{\phi}^{* \otimes 2}, \quad\left({ }_{d} \underline{\phi}^{* \wedge \wedge 2}\right) \mathcal{P}^{* \wedge 2}=\underline{\phi}^{* \wedge 2}
$$


on $\operatorname{Bil}(G, \mathbb{Q} / \mathbb{Z})$ and $\operatorname{Alt}(G, \mathbb{Q} / \mathbb{Z})$ are defined explicitly by the finite sums

$$
\left(\phi_{i}^{*} \otimes \phi_{j}^{*}\right)_{d}=\sum_{k, l \in I} p_{k i}^{*} p_{l j}^{*}\left(\phi_{k}^{\prime *} \otimes \phi_{l}^{\prime *}\right)_{d}, \quad\left(\phi_{i}^{*} \wedge \phi_{j}^{*}\right)_{d}=\sum_{k<l} p_{k l, i j}^{*}\left(\phi_{k}^{\prime *} \wedge \phi_{l}^{* *}\right)_{d},
$$

where $p_{k l, i j}^{*}=p_{k i}^{*} p_{l j}^{*}-p_{k j}^{*} p_{l i}^{*}$, and $p_{i j}^{*}=p_{j i} \in \mathbb{Z}_{\mathfrak{m}_{i}}$. Thus if $\boldsymbol{b} \in \operatorname{Bil}(G, \mathbb{Q} / \mathbb{Z})$ and $\boldsymbol{a} \in \operatorname{Alt}(G, \mathbb{Q} / \mathbb{Z})$ have matrix expressions $[\boldsymbol{b}]_{\underline{\phi}}=\left(b_{i j}\right)$ and $[\boldsymbol{a}]_{\underline{\phi}}=\left(a_{i j}\right)$ with respect to $\underline{\phi}$ and $[\boldsymbol{b}]_{\underline{\phi}^{\prime}}=\left(b_{i j}^{\prime}\right)$ and $[\boldsymbol{a}]_{\underline{\phi}^{\prime}}=\left(a_{i j}^{\prime}\right)$ with respect to $\underline{\phi}^{\prime}$, then $[\boldsymbol{b}]_{\underline{\phi}^{\prime}}=\mathcal{P}^{* \otimes 2}\left([\boldsymbol{b}]_{\underline{\phi}}\right)$ and $[\boldsymbol{a}]_{\underline{\phi}^{\prime}}=\overline{\mathcal{P}}^{* \wedge 2}\left([\boldsymbol{a}]_{\underline{\phi}}\right)$, and $b_{i j}^{\prime}=\sum_{k, l} p_{i k}^{*} p_{j l}^{*} b_{k l}$ and $a_{i j}^{\prime}=\sum_{k<l} p_{i j, k l}^{*} a_{k l}$.

Proof. Compute $\left(\phi_{i}^{*} \otimes \phi_{j}^{*}\right)_{d}\left(\phi_{k}^{\prime}, \phi_{l}^{\prime}\right)=\left(\phi_{i}^{*} \otimes \phi_{j}^{*}\right)_{d}\left(\prod_{i} \phi_{i}^{p_{i k}}, \prod_{j} \phi_{j}^{p_{j l}}\right)=p_{i k} p_{j l} / d_{i j}$. Since a basis change stabilizes $G$ 's homogeneous components, $d_{i j}=d_{k l}$ whenever $p_{i k}$ and $p_{j l}$ are nonzero, and then $\left(\phi_{i}^{*} \otimes \phi_{j}^{*}\right)_{d}\left(\phi_{k}^{\prime}, \phi_{l}^{\prime}\right)=p_{k i}^{*} p_{l j}^{*}\left(\phi_{k}^{\prime *} \otimes \phi_{l}^{\prime *}\right)_{d}\left(\phi_{k}^{\prime}, \phi_{l}^{\prime}\right)$. It follows that

$$
\left(\phi_{i}^{*} \otimes \phi_{j}^{*}\right)_{d}=\sum_{k, l} p_{k i}^{*} p_{l j}^{*}\left(\phi_{k}^{* *} \otimes \phi_{l}^{* *}\right)_{d}
$$

as desired. Similarly, in $\operatorname{Alt}(G, \mathbb{Q} / \mathbb{Z})$,

$$
\left(\phi_{i}^{*} \wedge \phi_{j}^{*}\right)_{d}\left(\phi_{k}^{\prime}, \phi_{l}^{\prime}\right)=\left(p_{i k} p_{j l}-p_{j k} p_{i l}\right)\left(\phi_{k}^{* *} \wedge \phi_{l}^{\prime *}\right)_{d}\left(\phi_{k}^{\prime}, \phi_{l}^{\prime}\right)
$$

The explicit computation of matrix expressions on $\operatorname{Bil}(G, \mathbb{Q} / \mathbb{Z})$ is immediately obtained as usual by setting $b_{i j}=n_{i j} / d_{i j}$ for all $i, j$, substituting the basis change formula into the expression $\boldsymbol{b}=\sum_{k, l} n_{k l}\left(\phi_{k}^{*} \otimes \phi_{l}^{*}\right)_{d}$, and then retrieving the coefficients of each $\left(\phi_{i}^{\prime *} \otimes \phi_{j}^{\prime *}\right)_{d}$. A similar computation yields the result for $\operatorname{Alt}(G, \mathbb{Q} / \mathbb{Z})$.

Example 5.8. Suppose $G=\widehat{\mathbb{Z}}^{n}$, with the standard basis $\phi=\left\{\phi_{1}, \ldots, \phi_{n}\right\}$. The coordinate expressions of $\boldsymbol{b} \in \operatorname{Bil}(G, \mathbb{Q} / \mathbb{Z})$ and $\boldsymbol{a} \in \operatorname{Alt}(G, \mathbb{Q} / \mathbb{Z})$ are matrices $\left(b_{i j}\right) \in$ $\mathrm{M}_{n}(\mathbb{Q} / \mathbb{Z})$ and $\left(a_{i j}\right) \in \operatorname{Alt}_{n}(\mathbb{Q} / \mathbb{Z})$, where $b_{i j}=\boldsymbol{b}\left(\phi_{i}, \phi_{j}\right)$ and $a_{i j}=\boldsymbol{a}\left(\phi_{i}, \phi_{j}\right)$. These matrices behave as bilinear forms on the coordinate expressions of elements of $G$ in the usual way. By Example 5.2, a basis change $\phi^{\prime}=\phi \mathcal{P}$ on $G$ is represented by a matrix $\mathcal{P}=\left(p_{i j}\right) \in \mathrm{GL}_{n}(\widehat{\mathbb{Z}})$, so that $\phi_{j}^{\prime}=\prod_{i} \phi_{i}^{p_{i j}}$. Let $\mathcal{P}^{*}=\left(p_{i j}^{*}\right)$ be the transpose of $\mathcal{P}$, so $p_{i j}^{*}=p_{j i}$. By Proposition 5.7, $\left(b_{i j}^{\prime}\right)=[\boldsymbol{b}]_{\underline{\phi}^{\prime}}=\mathcal{P} * \otimes 2\left([\boldsymbol{b}]_{\underline{\phi}}\right)$, and $\left(a_{i j}^{\prime}\right)=[\boldsymbol{a}]_{\underline{\phi^{\prime}}}=\mathcal{P}^{* \wedge 2}\left([\boldsymbol{a}]_{\underline{\phi}}\right)$, with

$$
b_{i j}^{\prime}=\sum_{1 \leq k, l \leq n} p_{k i}^{*} p_{l j}^{*} b_{k l}, \quad a_{i j}^{\prime}=\sum_{1 \leq k<l \leq n} p_{i j, k l}^{*} a_{k l}
$$

where $p_{i j, k l}^{*}=p_{i k}^{*} p_{j l}^{*}-p_{i l}^{*} p_{j k}^{*}$. Thus the matrix for $\boldsymbol{b}$ and for $\boldsymbol{a}$ in the new basis are computed by congruence transformation:

$$
[\boldsymbol{b}]_{\underline{\phi}^{\prime}}=\mathcal{P}^{*}\left([\boldsymbol{b}]_{\underline{\phi}}\right) \mathcal{P}, \quad[\boldsymbol{a}]_{\underline{\phi}^{\prime}}=\mathcal{P}^{*}\left([\boldsymbol{a}]_{\underline{\phi}}\right) \mathcal{P} .
$$

Alternatively, represent the matrix $\left(b_{i j}\right)=[\boldsymbol{b}]_{\underline{\phi}}$ by the $n^{2}$-tuple $\left(b_{11}, b_{12}, \ldots, b_{n n}\right) \in$ $(\mathbb{Q} / \mathbb{Z})^{n^{2}}$, ordered lexicographically. Then $\mathcal{P}^{* \otimes 2} \in \mathrm{GL}_{n^{2}}(\widehat{\mathbb{Z}})$ is the matrix whose $(i j, k l)$-entry is $p_{i k}^{*} p_{j l}^{*}$, and whose action on $n^{2}$-tuples is the usual one. Similarly, represent the matrix for $\boldsymbol{a} \in \operatorname{Alt}(G, \mathbb{Q} / \mathbb{Z})$ by the $\left(\begin{array}{l}n \\ 2\end{array}\right)$-tuple $\left(a_{12}, a_{13}, \ldots, a_{n-1 n}\right) \in$ $(\mathbb{Q} / \mathbb{Z})^{\left(\begin{array}{c}n \\ 2\end{array}\right)}$, and $\mathcal{P}^{* \wedge 2} \in \mathrm{GL}_{\left(\begin{array}{c}n \\ 2\end{array}\right)}(\widehat{\mathbb{Z}})$ is then the matrix whose $(i j, k l)$-entry is $p_{i j, k l}^{*}$, and whose action on $\left(\begin{array}{l}n \\ 2\end{array}\right)$-tuples is the usual one. 
Proposition 5.9. Suppose $G$ is a torsion-free proabelian group, with topological basis $\underline{\phi}=\left\{\phi_{i}\right\}_{i \in I}$, indexed by a linearly ordered set I. Then

$$
\operatorname{Bil}(G, \mathbb{Q} / \mathbb{Z}) \simeq \coprod_{i, j \in I} \mathrm{G}_{i j}^{*}, \quad \mathrm{H}^{2}(G, \mathbb{Q} / \mathbb{Z}) \simeq \operatorname{Alt}(G, \mathbb{Q} / \mathbb{Z}) \simeq \coprod_{i<j} \mathrm{G}_{i j}^{*}
$$

where, if $\mathfrak{m}_{k}=\operatorname{supp}\left(\left|\phi_{k}\right|\right)$ for $k \in I, \mathrm{G}_{i j}^{*}$ is the divisible group $\mathbb{Z}\left[\frac{1}{\mathfrak{m}_{i} \cap \mathfrak{m}_{j}}\right] / \mathbb{Z}$.

Proof. By Theorem $2.4, \mathrm{H}^{2}(G, \mathbb{Q} / \mathbb{Z}) \simeq \operatorname{Alt}(G, \mathbb{Q} / \mathbb{Z})$. Since $\underline{\phi}$ is a basis, $d \underline{\phi}^{* \otimes 2}$ and ${ }_{d} \phi^{* \wedge 2}$ are bases for ${ }_{d} \operatorname{Bil}(G, \mathbb{Q} / \mathbb{Z})$ and ${ }_{d} \operatorname{Alt}(G, \mathbb{Q} / \mathbb{Z})$ by Proposition 5.4 , and $\left|\left(\phi_{i}^{*} \otimes \phi_{j}^{*}\right)_{d}\right|=\left|\left(\phi_{i}^{*} \wedge \phi_{j}^{*}\right)_{d}\right|=d_{i j}$, where $d_{i j}=\operatorname{gcd}\left(d,\left|\phi_{i}\right|,\left|\phi_{j}\right|\right)$. Thus if

$$
{ }_{d} \mathrm{G}_{i j}^{*}=\frac{1}{d_{i j}} \mathbb{Z} / \mathbb{Z}={ }_{d}\left(\mathbb{Z}\left[\frac{1}{\mathfrak{m}_{i} \cap \mathfrak{m}_{j}}\right] / \mathbb{Z}\right),
$$

then

$$
{ }_{d} \operatorname{Bil}(G, \mathbb{Q} / \mathbb{Z}) \simeq \coprod_{i, j}{ }_{d} \mathrm{G}_{i j}^{*}
$$

and

$$
{ }_{d} \operatorname{Alt}(G, \mathbb{Q} / \mathbb{Z}) \simeq \coprod_{i<j} \mathrm{G}_{i j}^{*} .
$$

Since $\mathrm{G}_{i j}^{*}, \operatorname{Bil}(G, \mathbb{Q} / \mathbb{Z})$, and $\operatorname{Alt}(G, \mathbb{Q} / \mathbb{Z})$ are the union of their torsion subgroups, the result follows.

Example 5.10. If $G=\widehat{\mathbb{Z}}^{n}$, then $\mathrm{G}_{i j}^{*} \simeq \mathbb{Q} / \mathbb{Z}$, so

$$
\operatorname{Bil}(G, \mathbb{Q} / \mathbb{Z}) \simeq(\mathbb{Q} / \mathbb{Z})^{n^{2}} \simeq \mathrm{M}_{n}(\mathbb{Q} / \mathbb{Z}), \quad \operatorname{Alt}(G, \mathbb{Q} / \mathbb{Z}) \simeq(\mathbb{Q} / \mathbb{Z})^{\left(\begin{array}{l}
n \\
2
\end{array}\right)} \simeq \operatorname{Alt}_{n}(\mathbb{Q} / \mathbb{Z}) .
$$

Lemma 5.11. Suppose $G$ is a torsion-free proabelian group, and $\pi: G \rightarrow \bar{G}$ is a projection onto a torsion proabelian group $\bar{G}$. Then a topological basis $\underline{\phi}$ for $\bar{G}$ lifts to a topological basis $\underline{\phi}$ for $G$.

Proof. Since the proabelian group $\bar{G}$ is torsion, it is a product of finite cyclic groups, and $\exp (\bar{G})=r$ is finite. Let $\bar{\phi}=\left\{\bar{\phi}_{i}\right\}_{I}$ be a topological basis, and let $\bar{\phi}^{*}$ be its dual, a basis for $\bar{G}^{*}$. View $\bar{G}^{*}$ as a subgroup of $G^{*}$ via inflation. Let $H^{*}$ be a divisible hull of $\bar{G}^{*}$ in $G^{*}$. This exists by [F, Theorem 24.4]. Claim: there exists a system of bases $\underline{\phi}^{*}=\left\{{ }_{d} \underline{\phi}^{*}\right\}$ for $H^{*}$ extending $\underline{\phi}^{*}$, that is coherent in the sense of Lemma 5.3. Since $H^{*}$ is a direct sum of its homogeneous components, it suffices to assume $H^{*}$ is homogeneous. If $d$ is such that $r \mid d$ and $\operatorname{supp}(d)=\operatorname{supp}(r)$, then since $H^{*}$ is divisible there exist elements ${ }_{d} \phi_{i}^{*}$, each of order $d$, extending the basis elements $\bar{\phi}_{i}^{*}$, for each $i \in I$. These elements are easily seen to constitute a basis for ${ }_{d} H^{*}$, and the claim follows by induction on $d$. Since $H^{*}$ is divisible, it is a summand of $G^{*}$ by [K, Theorem 2]; let $\phi^{*}$ be an extension to all of $G^{*}$. Its dual $\phi$ is a topological basis for $G$ extending $\underline{\bar{\phi}}$. This completes the proof.

The next theorem shows that there is a standard form for any alternating form $\boldsymbol{a}$ on a torsion-free proabelian group $G$. This standard form is obtained by constructing a symplectic basis for $G$, essentially using the standard algorithm. When $G$ is homogeneous, for example when $G \simeq \widehat{\mathbb{Z}}^{n}$ or $G \simeq \mathbb{Z}_{p}^{n}$, this symplectic basis may be obtained from any given basis via basis change. The resulting 2-block-diagonal matrix representing $\boldsymbol{a}$ is then obtained from the original alternating matrix by congruence transformation. This is the crucial case since, as mentioned at the beginning of Section 5, any alternating form $\boldsymbol{a}$ over any torsion-free proabelian $G$ 
may be decomposed into a sum of its $p$-primary components, and each $p$-primary component is supported on the $p$-primary part of $G$, a (homogeneous) product of $\mathbb{Z}_{p}$ 's. Moreover, since $\boldsymbol{a}$ is continuous, its $p$-primary part is supported on a finite product of $\mathbb{Z}_{p}$ 's.

Theorem 5.12 "Symplectic Basis III". Suppose $G$ is a torsion-free proabelian group, and $\boldsymbol{a} \in \operatorname{Alt}(G, \mathbb{Q} / \mathbb{Z})$. Then $G$ has a basis $\underline{\psi}=\left\{\psi_{i}\right\}_{I}$ that is symplectic with respect to $\boldsymbol{a}$, so that for some subset $\left\{\psi_{1}, \ldots, \overline{\psi_{2 m}}\right\} \subset \underline{\psi}$, along with uniquely determined numbers $d_{i}: d_{i} \mid d_{i-1}$,

$$
\boldsymbol{a}\left(\psi_{2 i-1}, \psi_{2 i}\right)=-\boldsymbol{a}\left(\psi_{2 i}, \psi_{2 i-1}\right)=1 / d_{i}, \quad 1 \leq i \leq m,
$$

and $\boldsymbol{a}\left(\psi_{k}, \psi_{l}\right)=0$ otherwise. Thus $[\boldsymbol{a}]_{\underline{\psi}}$ is an alternating 2-block-diagonal matrix. In basis notation,

$$
\boldsymbol{a}=\sum_{i=1}^{m}\left(\psi_{2 i-1}^{*} \wedge \psi_{2 i}^{*}\right)_{d_{i}}
$$

If $G$ has a homogeneous basis $\underline{\phi}$, then $\underline{\psi}$ is obtainable in the standard way by a basis change $\underline{\psi}=\underline{\phi} \mathcal{P}$, and $[\boldsymbol{a}]_{\underline{\psi}}=\overline{\mathcal{P}}^{* \wedge 2}[\boldsymbol{a}]_{\underline{\phi}}$ is the resulting alternating 2-block-diagonal matrix for $\boldsymbol{a}$ with respect to $\underline{\psi}$.

Proof. Let $r=\exp (\boldsymbol{a})$; then $\boldsymbol{a} \in \operatorname{Alt}\left(G / G^{r}, \mathbb{Q} / \mathbb{Z}\right)$. Since $G$ is torsion-free, $G / G^{r}$ has homogeneous primary components. By continuity, $\boldsymbol{a}$ is supported on a finite summand of $G / G^{r}$. Therefore it may be assumed that $G / G^{r}$ is finite, and Lemma 3.8 applies. By Lemma 5.11, the resulting symplectic basis on $G / G^{r}$ lifts to $G$. This proves the first part.

Suppose $G$ is homogeneous, with $G \simeq \mathbb{Z}_{\mathfrak{m}}^{c}$ for some $\mathfrak{m} \subset \mathcal{P}$ and cardinal number $c$. Since $\boldsymbol{a}$ is continuous, it may be assumed that $c$ is finite. Set $r=\exp (\boldsymbol{a})$. Since $r \in \mathbb{N}(\mathfrak{m}), G / G^{r} \simeq(\mathbb{Z} / r \mathbb{Z})^{c}$, and Lemma 3.4 applies. Let $\bar{\phi}$ be the image of $\underline{\phi}$ in $G / G^{r}$, and let $\bar{\psi}=\bar{\phi} \mathcal{P}_{r}$, where $\mathcal{P}_{r} \in \mathrm{GL}_{c}(\mathbb{Z} / r \mathbb{Z})$, be a symplectic basis for $\bar{G} / G^{r}$ with respect to $\overline{\boldsymbol{a}}$. Extend $\bar{\psi}$ to the $\operatorname{supp}(r)$-primary part of $G$ using Lemma 5.11. Let $\mathfrak{m}^{\prime}=\operatorname{supp}(r)$. By the proof of Lemma 5.11, the extended set is a homogeneous basis for the $\mathfrak{m}^{\prime}$-primary summand of $G$. Let $\psi$ be the homogeneous basis for $G$ obtained by multiplying this basis "diagonally" with a homogeneous basis for the $\mathfrak{m} \backslash \mathfrak{m}^{\prime}$-primary part of $G$. That is, if $\mathrm{G}_{i}$ is a procyclic component of the homogeneous decomposition of $G, \phi_{i}$ is a basis element for the $\mathrm{G}_{i}\left(\mathfrak{m}^{\prime}\right)$, and $\phi_{i}^{\prime}$ is a basis element for $\mathrm{G}_{i}\left(\mathfrak{m} \backslash \mathfrak{m}^{\prime}\right)$, then let $\psi_{i}=\phi_{i} \phi_{i}^{\prime}$. Then $\underline{\psi}=\phi \mathcal{P}$ for some basis change $\mathcal{P}$, extending the basis change $\overline{\bar{\psi}}=\bar{\phi} \mathcal{P}_{r}$ in $G / G^{r}$, and $\overline{\underline{\psi}}$ is symplectic with respect to $\boldsymbol{a}$. The last statement follows by Proposition 5.7.

\section{Bases in $F^{\times}$}

To transport the basis computations from $\operatorname{Alt}(G, \mathbb{Q} / \mathbb{Z})$ to the Brauer group $\operatorname{Br}(F)$, some notion of basis must be defined for $F^{\times}$which is dual to the notion of basis for the torsion-free group $G$. These "bases" for $F^{\times}$will be constructed from the Kummer map $\kappa_{\omega_{d}}$ of (4.1), between $d$-torsion subgroups of $G^{*}$ and $d$-torsion quotients $F^{\times} / F^{\times d}$.

As usual, assume $F$ is a field of characteristic 0 that contains the roots of unity $\mu$. Let $\omega: \mu \rightarrow \mathbb{Q} / \mathbb{Z}$ be a fixed identification. Let $\underline{\phi}=\left\{\phi_{i}\right\}_{I}$ be a (topological) basis for a torsion-free abelian Galois group $\mathrm{G}_{\Omega / F}$, and let $\underline{\phi}^{*}$ stand for the family 
of dual bases ${ }_{d} \phi^{*}=\left\{{ }_{d} \phi_{i}^{*}\right\}_{I}$ for ${ }_{d} \mathrm{G}_{\Omega / F}^{*}$, for each $d \in \mathbb{N}$. The choice of $\omega$ determines a corresponding family of Kummer isomorphisms $\kappa_{\omega_{d}}:{ }_{d} \mathrm{G}_{\Omega / F}^{*} \rightarrow \Omega^{d} \cap F^{\times} / F^{\times d}$.

Definition 5.13. For all $d \in \mathbb{N}$, define the Kummer dual of the basis $\phi$ to be the basis $d \underline{x}=\kappa_{\omega_{d}}\left(d \underline{\phi}^{*}\right) \subset\left(\Omega^{d} \cap F^{\times}\right) / F^{\times d}$ dual to $\underline{\phi}$ via Kummer theory and the choice $\omega$. By Definition 5.1c, $\left\langle\phi_{i},{ }_{d} x_{j}\right\rangle_{d}=\omega_{d_{j}}^{\delta_{i j}}$, for all $\bar{i}, j \in I$, where $d_{j}=\left(d,\left|\phi_{j}\right|\right)=\left|{ }_{d} \phi_{j}^{*}\right|$.

By Definition 5.1c, the Kummer transpose of a topological basis change $\underline{\phi}^{\prime}=\phi \mathcal{P}$, where $\mathcal{P}=\left(p_{i j}\right)$, is given by $d \underline{x}^{\prime} \mathcal{P}^{*}=d \underline{x}$. Explicitly,

$$
{ }_{d} x_{j}=\prod_{i}\left({ }_{d} x_{i}^{\prime}\right)^{p_{i j}^{*}},
$$

where $p_{i j}^{*}=p_{j i}$ is interpreted $\left(\bmod \left|{ }_{d} x_{i}\right|\right)$. Note that for fixed $j$, almost all of the $p_{i j}^{*}$ are zero, so that the product is finite. Moreover, if $p_{i j}^{*} \neq 0$, then in the notation of Definition 5.1a, $i$ and $j$ are in the same homogeneous component $\mathrm{G}_{j}^{\left|I_{j}\right|}$ of $G$, so that by Definition $5.1 \mathrm{c}, p_{i j}^{*}$ may be taken to lie in $\mathbb{Z}_{\mathfrak{m}_{i}}$.

The following lemma is a Kummer theory version of Lemma 5.3.

Lemma 5.14. Suppose $\mathrm{G}_{\Omega / F}$ is homogeneous, with $\mathrm{G}_{\Omega / F} \simeq \mathbb{Z}_{\mathfrak{m}}^{c}$ for some $\mathfrak{m} \subset \mathcal{P}$ and some cardinal number $c$. Let $\phi$ be a homogeneous basis with Kummer dual bases $d \underline{x}$, for all $d \in \mathbb{N}$. Then for all $d, e \in \mathbb{N}: d \mid e$ and $e / d \in \mathbb{N}(\mathfrak{m}), d \underline{x}$ is the image of $e \underline{x}$ under the natural projection map $F^{\times} / F^{\times} e \rightarrow F^{\times} / F^{\times} d$. Conversely, suppose $\mathfrak{m} \subset \mathcal{P}$, and for all $d \in \mathbb{N}(\mathfrak{m})$, $d \underline{x}$ is a homogeneous pure independent set of exponent $d$ in $F^{\times} / F^{\times} d$, such that the family of bases $\{d \underline{x}: d \in \mathbb{N}(\mathfrak{m})\}$ is coherent under the natural projections. Then there exists a Galois extension $\Omega / F$ with torsion-free homogeneous abelian group $\mathrm{G}_{\Omega / F} \simeq \mathbb{Z}_{\mathfrak{m}}^{c}$ and topological basis $\phi$, such that $d \underline{x}$ is the Kummer dual of $\phi$, for all $d \in \mathbb{N}(\mathfrak{m})$. Explicitly, $\Omega$ is the composite of the Kummer extensions defined by the subgroups $\left\langle{ }_{d} \underline{x}\right\rangle \leq F^{\times} / F^{\times d}$.

Proof. Let $d^{\prime}, e^{\prime}$ be the parts of $d$ and $e$ supported on $\mathfrak{m}$; then since $e / d \in \mathbb{N}(\mathfrak{m})$, $e / d=e^{\prime} / d^{\prime}$. By Lemma $5.3,{ }_{d} \underline{\phi}^{*}=(e / d)_{e} \underline{\phi}^{*}$, therefore by the definition of $\kappa_{\omega_{d}}$ and $\kappa_{\omega_{e}},\left\langle-{ }_{e} x_{j}\right\rangle_{e}^{e / d}=\left\langle-,{ }_{d} x_{j}\right\rangle_{d}$. On the other hand, since $e / d=e^{\prime} / d^{\prime},\left\langle-,{ }_{e} x_{j}\right\rangle_{e}^{e / d}=$ $\left\langle-,{ }_{e} x_{j}\right\rangle_{d}$. Therefore ${ }_{e} x_{j} \equiv{ }_{d} x_{j}\left(\bmod F^{\times d}\right)$. This proves the first statement.

Conversely, suppose the elements in the set $\left\{d \underline{x} \subset F^{\times} / F^{\times} d: d \in \mathbb{N}(\mathfrak{m})\right\}$ are coherent under the natural projections. Let ${ }_{d} \underline{\phi}^{*}$ be the corresponding characters of $\mathrm{G}_{F}$. Since $d \underline{x}$ is a homogeneous pure independent set of exponent $d$, so is ${ }_{d} \underline{\phi}^{*}$, hence each ${ }_{d} \phi^{*}$ is a basis for a summand of ${ }_{d} \mathrm{G}_{F}^{*}$. Since $e / d \in \mathbb{N}(\mathfrak{m}),\left\langle-{ }_{e} x_{j}\right\rangle_{d}^{-}=$ $\left\langle-,{ }_{e} x_{j}\right\rangle_{e}^{e / d}$, and so ${ }_{d} \phi^{*}=(e / d)_{e} \underline{\phi}^{*}$. That is, the ${ }_{d} \phi^{*}$ form a coherent set. By Lemma 5.3, there exists a Galois group $\mathrm{G}_{\Omega / F} \simeq \mathbb{Z}_{\mathfrak{m}}^{c}$ with a topological basis $\underline{\phi}=$ $\left\{\phi_{i}\right\}_{I}$ whose dual basis is the ${ }_{d} \phi^{*}$. Thus $\left\langle\phi_{i},{ }_{d} x_{j}\right\rangle_{d}=\omega_{d}^{\delta_{i j}}$, for all $i, j \in I$, and $d \in \mathbb{N}(\mathfrak{m})$. Clearly $\Omega$ is the composite of the Kummer extensions defined by the $d \underline{x}$, so the last statement follows.

Definition 5.15. In the situation of Lemma 5.14, let $x_{i}=\varliminf_{\lim _{d}}\left({ }_{d} x_{i}\right)$ for each $i$, where $d \in \mathbb{N}(\mathfrak{m})$, and set $\underline{x}=\left\{x_{i}\right\}_{I}$. This is a subset of the closed subgroup $\lim _{d}\left(\Omega^{d} \cap F^{\times} / F^{\times} d\right)$ of the $\mathfrak{m}$-adic completion $F_{\mathfrak{m}}^{\times}$of $F^{\times}$. Write

$$
F\left(\underline{x}^{1 / \mathfrak{m}^{\infty}}\right)=\underset{d \in \underset{\mathbb{N}(\mathfrak{m})}{\lim }}{\longrightarrow} F\left(\left\{x_{i}^{1 / d}\right\}_{I}\right)
$$


for this field extension. If $\underline{\psi}=\phi \mathcal{P}$ is a topological basis change, and $\underline{\psi}$ and $\underline{\phi}$ correspond to $\underline{y}$ and $\underline{x}$, respectively, write

$$
\underline{y}^{*}=\underline{x}
$$

for the induced basis change.

Without the machinery of inverse limits, the basis change expression can be viewed as shorthand for the family of basis changes $\left\{{ }_{d} \underline{\mathcal{P}}^{*}={ }_{d} \underline{x}\right\}$.

Remark 5.16. The following special case is particularly interesting. Suppose $\underline{x} \subset$ $F^{\times}$is an $\mathfrak{m}$-independent subset of $F^{\times}$. For example, $\underline{x}$ could be part of a transcendence basis for $F$ over some subfield of $F$, or a set of nonassociate prime elements in a ring with quotient field $F$. Then $\Omega=F\left(\underline{x}^{1 / \mathfrak{m}^{\infty}}\right)$ is the composite of all radical extensions of degree $d \in \mathbb{N}(\mathfrak{m})$ defined by $\underline{x}$. By the above, the Galois group $\mathrm{G}_{\Omega / F} \simeq \mathbb{Z}_{\mathfrak{m}}^{c}$ has a homogeneous basis $\underline{\phi}$, such that $\left\langle\phi_{i}, x_{j}\right\rangle_{d}=\omega_{d}^{\delta_{i j}}$.

Generators in $\operatorname{Br}(F)$

The basis elements $d \underline{x}=\left\{{ }_{d} x_{i}\right\}$ for the various $F^{\times} / F^{\times} d$ suggest an obvious generating set for part of $\operatorname{Br}(F)$, namely the set of symbol classes that are expressible in terms of the ${ }_{d} x_{i}$. These elements will be used to import the explicit basis calculations on $\operatorname{Alt}(G, \mathbb{Q} / \mathbb{Z})$ into $\operatorname{Br}(F)$.

Assume $F$ contains $\mu$, and $\mathrm{G}_{\Omega / F}$ is a torsion-free abelian Galois group, with topological basis $\underline{\phi}=\left\{\phi_{i}\right\}_{I}$, for some linearly ordered set $I$. By Proposition 5.4, the set ${ }_{d} \underline{\phi}^{* \wedge 2}=\left\{\left(\phi_{i}^{*} \wedge \phi_{j}^{*}\right)_{d}: i<j \in I\right\}$ is a basis for $\operatorname{Alt}\left(\mathrm{G}_{\Omega / F}, \mathbb{Q} / \mathbb{Z}\right)$. Let $d \underline{x}=$ $\kappa_{\omega_{d}}\left({ }_{d} \phi^{*}\right)$ be the Kummer dual, as in Definition 5.13. Assume for simplicity that $\mathrm{G}_{\Omega / F}$ is homogeneous, so $\mathrm{G}_{\Omega / F} \simeq \mathbb{Z}_{\mathfrak{m}}^{c}$ for some $\mathfrak{m} \subset \mathcal{P}$ and some cardinal number $c$. The general case can be reduced to this situation using the primary decomposition. By Theorem 4.4, the elements $\left[\left(x_{i}, x_{j}\right)_{\omega_{d}}\right]$, where $d \in \mathbb{N}(\mathfrak{m})$, generate ${ }_{d} \operatorname{Br}^{\circ}(\Omega / F)$. If $\kappa^{\wedge 2}$ is injective, then they form a basis for ${ }_{d} \mathrm{Br}^{\circ}(\Omega / F)$.

Theorem 5.17 "Disjoint Reduced Symbol Presentation II". Assume $\mu \subset F$. Suppose $\Omega / F$ is a Galois extension with torsion-free abelian group $\mathrm{G}_{\Omega / F}$, and $\boldsymbol{\xi} \in$ $\operatorname{Br}^{\circ}(\Omega / F)$. Then $\mathrm{G}_{\Omega / F}$ has a basis $\underline{\psi}$ such that for some subset $\left\{\psi_{1}, \ldots, \psi_{2 m}\right\} \subset \underline{\psi}$, along with numbers $d_{i}: d_{i} \mid d_{i-1}, \boldsymbol{\xi} \bar{h}$ as a disjoint reduced symbol presentation

$$
\boldsymbol{\xi}=\sum_{i=1}^{m}\left[\left(y_{2 i-1}, y_{2 i}\right)_{\omega_{d_{i}}}\right]
$$

where $y_{2 i-1}=\kappa_{\omega_{d_{i}}}\left(d_{i} \psi_{2 i-1}^{*}\right)$ and $y_{2 i}=\kappa_{\omega_{d_{i}}}\left(d_{i} \psi_{2 i}^{*}\right)$. If $\kappa^{\wedge 2}$ is injective, the $d_{i}$ are uniquely determined.

If $\mathrm{G}_{\Omega / F}$ is homogeneous, with homogeneous topological basis $\underline{\phi}=\left\{\phi_{i}\right\}_{I}$, then the basis $\underline{\psi}$ is obtainable through a basis change $\underline{\psi}=\underline{\phi} \mathcal{P}$. More precisely, suppose the Kummer dual of $\underline{\phi}$ is $\underline{x}=\left\{x_{i}\right\}_{I}$, and

$$
\boldsymbol{\xi}=\sum_{1 \leq i<j \leq n}\left[\left(x_{i}, x_{j}\right)_{\omega_{r_{i j}}}^{s_{i j}}\right]
$$

Set $a_{i j}=-a_{j i}=s_{i j} / r_{i j} ;$ then $[\boldsymbol{a}]_{\phi}=\left(a_{i j}\right) \in \operatorname{Alt}_{n}(\mathbb{Q} / \mathbb{Z})$ represents $\boldsymbol{\xi}$. Let $\underline{\psi}=\phi \mathcal{P}$ be a symplectic basis for $\mathrm{G}_{\Omega / F}$ with respect to the form $\boldsymbol{a}$, and let $\underline{y}=\left\{y_{i}\right\}_{I}$ be the 
Kummer dual of $\underline{\psi}$, so that $\underline{y}=\underline{x} Q^{*}$, where $Q=\mathcal{P}^{-1}$. Then

$$
\boldsymbol{\xi}=\sum_{i=1}^{m}\left[\left(y_{2 i-1}, y_{2 i}\right)_{\omega_{d_{i}}}\right]
$$

is a disjoint reduced symbol presentation.

Proof. The proof follows immediately by Theorem 4.9, Theorem 5.12, and Lemma 5.14 .

Corollary 5.18. Let $F=\mathbb{C}\left(\left(x_{1}\right)\right) \cdots\left(\left(x_{n}\right)\right)$. Then for each $d \in \mathbb{N},\left\{\left[\left(x_{i}, x_{j}\right)_{\omega_{d}}\right]\right.$ :

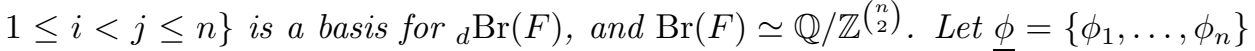
be the topological basis for $\mathrm{G}_{F}^{\mathrm{ab}}$ Kummer dual to $\underline{x}=\left\{x_{1}, \ldots, x_{n}\right\}$ via $\omega$. Then the map

$$
\begin{aligned}
\kappa^{\wedge 2}: \operatorname{Alt}\left(\mathrm{G}_{F}, \mu\right) & \stackrel{\sim}{\longrightarrow} \operatorname{Br}(F) \\
\omega_{*}\left(\phi_{i}^{*} \wedge \phi_{j}^{*}\right)_{d} & \longmapsto\left[\left(x_{i}, x_{j}\right)_{\omega_{d}}\right]
\end{aligned}
$$

is a natural isomorphism.

Proof. The first statement is immediate from the third by Proposition 5.4. The iterated henselian valuation on $F$ defines a decomposition $F^{\times} \simeq \mathbb{Z}^{n} \times U$, where $U$ is divisible. Clearly the image of $\underline{x}$ in $F^{\times} / F^{\times d}$ is a basis for all $d, \underline{\phi}$ is a basis for $G_{F}^{\mathrm{ab}}$, and $\mathrm{G}_{F}^{\mathrm{ab}} \simeq \widehat{\mathbb{Z}}^{n}$. Therefore the second statement follows from the third by Example 5.10.

It can be shown with a little valuation theory that every finite extension of $F$ is radical, hence $\mathrm{G}_{F}$ is abelian. Therefore $\operatorname{Alt}\left(\mathrm{G}_{F}^{\mathrm{ab}}, \mu\right) \simeq \mathrm{H}^{2}\left(\mathrm{G}_{F}^{\mathrm{ab}}, \mu\right)=\mathrm{H}^{2}\left(\mathrm{G}_{F}, \mu\right)$. By Hilbert's Theorem 90 and the long exact cohomology sequence associated with the Kummer sequence

$$
1 \rightarrow \mu_{d} \rightarrow F_{\text {alg }}^{\times} \rightarrow F_{\text {alg }}^{\times} \rightarrow 1,
$$

$\mathrm{H}^{2}\left(\mathrm{G}_{F}, \mu_{d}\right) \simeq{ }_{d} \operatorname{Br}(F)$. It follows that $\mathrm{H}^{2}\left(\mathrm{G}_{F}, \mu\right)=\lim _{d} \mathrm{H}^{2}\left(\mathrm{G}_{F}, \mu_{d}\right) \simeq \lim _{d}\left({ }_{d} \operatorname{Br}(F)\right)$ $=\operatorname{Br}(F)$. By Theorem 4.4, the composite of these maps yields the natural map $\operatorname{Alt}\left(\mathrm{G}_{F}^{\mathrm{ab}}, \mu\right) \rightarrow \operatorname{Br}(F)$ given by $\kappa^{\wedge 2} \cdot \omega_{*}^{-1}$, an isomorphism that sends $\omega_{*}\left(\phi_{i}^{*} \wedge \phi_{j}^{*}\right)_{d}$ to $\left[\left(x_{i}, x_{j}\right)_{\omega_{d}}\right]$. This completes the proof.

Example 5.19. The inverse of $\kappa^{\wedge 2}$ in Corollary 5.18 is analogous to the Hasse invariant in local class field theory. Suppose $F$ is a local field, which is by definition a complete discretely valued field with finite residue field. The Hasse invariant map in local class field theory is an isomorphism

$$
\operatorname{inv}_{F}: \operatorname{Br}(F) \rightarrow \mathbb{Q} / \mathbb{Z} .
$$

Explicitly, every Brauer class $\boldsymbol{\xi}$ is represented by a cyclic algebra $\left[\left({ }_{d} \phi^{*}, \pi^{c}\right)\right]$, where ${ }_{d} \phi^{*}$ is the Frobenius character of order $d$, defined on a Frobenius generator $\phi$ in

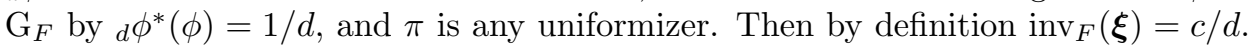
The map's naturality relies on the naturality of both the Frobenius map and the valuation defined by $\pi$.

Let $F=\mathbb{C}\left(\left(x_{1}\right)\right) \cdots\left(\left(x_{n}\right)\right)$. By Corollary 5.18, a choice of $\omega$, together with the choice of the ordered set $\{\underline{x}\}$, determines an isomorphism

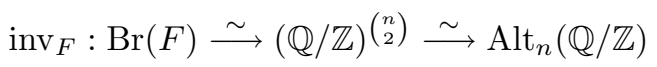


sending $\left[\left(x_{i}, x_{j}\right)_{\omega_{d}}^{c}\right]$ to the $i j$-th standard generator $(0, \ldots, 0, c / d, 0, \ldots, 0)$ corresponding to the element $c\left(\phi_{i}^{*} \wedge \phi_{j}^{*}\right)_{d}$. In particular, when $n=2$ there is an isomorphism $\operatorname{Br}(F) \simeq \mathbb{Q} / \mathbb{Z}$, analogous to the Hasse invariant map. In Example 6.5 below it is shown how $\operatorname{Alt}_{n}(\mathbb{Q} / \mathbb{Z})$, hence $(\mathbb{Q} / \mathbb{Z})\left(\begin{array}{l}n \\ 2\end{array}\right)$, transforms under restriction and corestriction. In the $n=2$ case, the usual formulas of local class field theory are obtained.

\section{Computing Functorial maps}

This section contains computations of inflation, restriction, and corestriction on bilinear forms, cohomology, and alternating forms with coefficients in $\mathbb{Q} / \mathbb{Z}$, and finally on the Brauer group of a field that contains $\mu$.

Setup 6.1. Suppose there is an exact sequence of proabelian groups

$$
1 \longrightarrow H \stackrel{\iota}{\longrightarrow} G \stackrel{\pi}{\longrightarrow} \bar{G} \longrightarrow 1
$$

where $G$ is torsion-free and $\bar{G}$ has finite exponent $r$. For each $d$ divisible by $r$, let $\jmath_{d}$ denote the composite of exponentiation by $d$ and $\iota^{-1}$,

$$
\jmath_{d}: G \longrightarrow H \text {. }
$$

Recall $\iota^{*}$ is restriction, $\pi^{*}$ is inflation, and the composition $\jmath_{d}^{*} \cdot \iota^{*}: G^{*} \rightarrow G^{*}$ is multiplication by $d$. If $\iota$ is open and $s=|\bar{G}|$, then $\jmath=\jmath_{s}$ is the transfer, and $\jmath^{*}$ is corestriction.

Definitions 6.2. Assume Setup 6.1.

(a) Decompositions $\Psi: \prod_{J} \mathbb{Z}_{\mathfrak{m}_{j}}^{b_{j}} \stackrel{\sim}{\longrightarrow} H$ and $\Phi: \prod_{J} \mathbb{Z}_{\mathfrak{m}_{j}}^{c_{j}} \stackrel{\sim}{\longrightarrow} G$ and the corresponding bases $\underline{\psi}$ and $\underline{\phi}$ are compatible if $\iota\left(\Psi\left(\mathbb{Z}_{\mathfrak{m}_{j}}^{b_{j}}\right)\right) \leq \Phi\left(\mathbb{Z}_{\mathfrak{m}_{j}}^{c_{j}}\right)$, for all $j \in J$.

(b) If $\underline{\psi}$ and $\underline{\phi}$ are compatible, write $\mathcal{P}=\left(p_{i j}\right)=[\iota]_{\underline{\underline{\psi}}}$, meaning that for each $\psi_{j} \in$ $\Psi\left(\mathbb{Z}_{\mathfrak{m}_{k}}^{b_{k}}\right), \psi_{j}=\prod_{i} \phi_{i}^{p_{i j}}$, where $\phi_{i} \in \Phi\left(\mathbb{Z}_{\mathfrak{m}_{k}}^{c_{k}}\right)$ and $p_{i j} \in \mathbb{Z}_{\mathfrak{m}_{k}}$. Let $\mathcal{P}^{*}=\left[\iota^{*}\right]_{\underline{\phi}^{*}}$ stand for the family of coordinate representations $\left[\iota^{*}\right]_{d}^{d} \underline{\psi}^{*} \underline{\phi}^{*}:{ }_{d} G^{*} \rightarrow{ }_{d} H^{*}$. Thus $\iota^{*}\left({ }_{d} \phi_{j}^{*}\right)=$ $\sum_{i \in I} p_{i j}^{*}\left({ }_{d} \psi_{i}^{*}\right)$. If $\underline{y}$ and $\underline{x}$ are the respective Kummer bases as in Definition 5.15, let $\mathcal{P}^{*}=\left[\iota^{*}\right] \underline{\underline{x}}$ denote the corresponding family of coordinate representations of the Kummer transpose of $\iota^{*}$. Apply similar notation to maps on bilinear forms and alternating forms.

Compatible decompositions always exist. For example, the primary decompositions for $H$ and $G$ are obviously compatible.

Lemma 6.3. Assume Setup 6.1.

(a) $H \simeq G$. In particular, if $G$ has a homogeneous decomposition, then $H$ has a compatible homogeneous decomposition.

(b) $\iota$ is diagonalizable. Specifically, let $\underline{\psi}=\left\{\psi_{i}\right\}_{I}$ and $\underline{\phi}=\left\{\phi_{i}\right\}_{I}$ be compatible bases. Then there exist basis changes $\underline{\psi}^{\prime}=\underline{\psi} \underline{\mathcal{C}}$ and $\underline{\phi}^{\prime}=\underline{\phi}^{-1}$, such that for some numbers $d_{i} \in \mathbb{N}, \iota\left(\psi_{i}^{\prime}\right)=\phi_{i}^{\prime d_{i}}$ for each $i \in I$. Thus if $[\iota]_{\underline{\underline{\psi}}}^{\phi}=\mathcal{P}$, then $[\iota]_{\underline{\psi^{\prime}}}^{\phi^{\prime}}=\mathcal{B P C}=$ $\mathcal{D}=\operatorname{diag}\left\{d_{i}\right\}_{I}$.

Proof. View $H$ as a closed subgroup of $G$. To prove $H \simeq G$ it suffices to show $H$ and $G$ have isomorphic $p$-primary components for all $p$. Therefore assume $H \simeq \mathbb{Z}_{p}^{b_{p}}$ 
and $G \simeq \mathbb{Z}_{p}^{c_{p}}$. Consider the commutative diagram

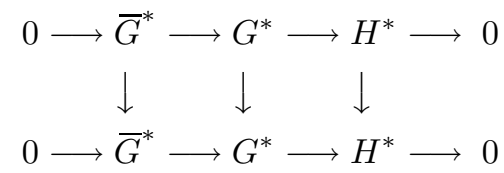

where the vertical arrows are multiplication by $p$. By the Snake Lemma, the sequence of $\mathbb{Z} / p \mathbb{Z}$-vector spaces

$$
0 \longrightarrow{ }_{p} \bar{G}^{*} \longrightarrow{ }_{p} G^{*} \stackrel{\pi^{*}}{\longrightarrow}{ }_{p} H^{*} \longrightarrow \bar{G}^{*} / p \bar{G}^{*} \longrightarrow G^{*} / p G^{*} \longrightarrow 0
$$

is exact. Since $G^{*}$ is divisible, $G^{*} / p G^{*}=\{0\}$. Since these are all vector spaces, there are direct sum decompositions

$$
\begin{aligned}
{ }_{p} G^{*} & \simeq{ }_{p} \bar{G}^{*} \oplus \pi^{*}\left({ }_{p} G^{*}\right), \\
{ }_{p} H^{*} & \simeq \bar{G}^{*} / p \bar{G}^{*} \oplus \pi^{*}\left({ }_{p} G^{*}\right) .
\end{aligned}
$$

Now compare $\mathbb{Z} / p \mathbb{Z}$-dimensions. Since $\bar{G}^{*}$ has bounded order, it is a direct sum of (finite) cyclic $p$-groups, and so $\operatorname{dim}\left(\bar{G}^{*} / p \bar{G}^{*}\right) \leq \operatorname{rank}\left(\bar{G}^{*}\right)=\operatorname{dim}\left({ }_{p} \bar{G}^{*}\right)$. Therefore

$$
\begin{aligned}
\operatorname{dim}\left({ }_{p} H^{*}\right) & =\operatorname{dim}\left(\bar{G}^{*} / p \bar{G}^{*}\right)+\operatorname{dim}\left(\pi^{*}\left({ }_{p} G^{*}\right)\right) \\
& \leq \operatorname{dim}\left({ }_{p} \bar{G}^{*}\right)+\operatorname{dim}\left(\pi^{*}\left({ }_{p} G^{*}\right)\right)=\operatorname{dim}\left({ }_{p} G^{*}\right) .
\end{aligned}
$$

Thus $b_{p} \leq c_{p}$. On the other hand, since $\iota$ 's cokernel has exponent $r, G^{r} \leq H$. Since $r \mathbb{Z}_{p} \simeq \mathbb{Z}_{p}$ as $\mathbb{Z}_{p}$-modules, $G^{r} \simeq \mathbb{Z}_{p}^{c_{p}}$, therefore $c_{p} \leq b_{p}$. Thus $b_{p}=c_{p}$. Since a map of proabelian groups takes $p$-primary components into $p$-primary components, the primary decompositions of $H$ and $G$ are compatible. This proves (a).

To prove (b) it may be assumed that $H \simeq G \simeq \mathbb{Z}_{\mathfrak{m}}^{c}$ for some $\mathfrak{m} \subset \mathcal{P}$ and some cardinal number $c=|I|$. Since any two bases of $G$ are then related by a basis change, it suffices to show there exists a topological basis $\phi=\left\{\phi_{i}\right\}_{I}$ for $G$ such that for some numbers $d_{i}, \psi=\left\{\phi_{i}^{d_{i}}\right\}_{I}$ is a topological basis for $H$.

Let $\phi$ be the topological basis for $G$ extending a basis $\bar{\phi}$ for $\bar{G}$, constructed in Lemma 5.11. Set $d_{i}=\left|\bar{\phi}_{i}\right|$. Claim: $\underline{\psi}=\left\{\phi_{i}^{d_{i}}\right\}_{I}$ is a topological basis for H. Since "*" is an exact functor, the sequence

$$
0 \longrightarrow \bar{G}^{*} \longrightarrow G^{*} \longrightarrow H^{*} \longrightarrow 0
$$

is exact. Suppose $\tau \in G$. Since $\underline{\phi}$ is a basis, $\tau=\prod_{I} \phi_{i}^{g_{i}}$, for some $g_{i} \in \mathbb{Z}_{\mathfrak{m}}$. Since $\bar{\phi}$ is a basis for $\bar{G}$, by exactness $\bar{\tau} \in H$ if and only if $\bar{\phi}_{j}^{*}(\tau)=0$ for all $j$, i.e., if and only if $d_{i} \mid g_{i}$ for all $i \in I$. Thus $\psi$ is a topological basis for $H$. This proves the claim, and completes the proof.

Computing Functorial Maps in $\operatorname{Bil}(G, \mathbb{Q} / \mathbb{Z})$ and $\operatorname{Alt}(G, \mathbb{Q} / \mathbb{Z})$

Theorem 6.4. Assume Setup 6.1. Let $\underline{\phi}=\left\{\phi_{i}\right\}_{I}$ and $\underline{\psi}=\left\{\psi_{i}\right\}_{I}$ be compatible bases for $G$ and $H$ with respect to $\iota$, indexed by a linearly ordered set $I$, and let $\mathcal{P}=\left(p_{i j}\right)=[\iota] \frac{\phi}{\underline{\mathcal{L}}}$.

(a) Let $\mathcal{B P \mathcal { C }}=\mathcal{D}=\operatorname{diag}\left\{d_{i}\right\}_{I}$ be a diagonalization of $\mathcal{P}$ as in Lemma 6.3 , and set $\mathcal{B}=\left(b_{i j}\right)$. Then the kernel of the restriction map on $\operatorname{Bil}(G, \mathbb{Q} / \mathbb{Z})$ and $\operatorname{Alt}(G, \mathbb{Q} / \mathbb{Z})$, respectively, have bases

$$
\left\{\sum_{k, l} b_{k i}^{*} b_{l j}^{*}\left(\phi_{k}^{*} \otimes \phi_{l}^{*}\right)_{d_{i j}}: i, j \in I\right\}, \quad\left\{\sum_{k<l} b_{k l, i j}^{*}\left(\phi_{k}^{*} \wedge \phi_{l}^{*}\right)_{d_{i j}}: i<j \in I\right\},
$$


where $d_{i j}=d_{i} d_{j}, \mathcal{B}^{*}=\left(b_{i j}^{*}\right)$ and $b_{k l, i j}^{*}=b_{k i}^{*} b_{l j}^{*}-b_{l i}^{*} b_{k j}^{*}$.

(b) The coordinate expressions of the restriction maps are

$$
\begin{aligned}
\mathcal{P}^{* \otimes 2}: \mathrm{M}_{|\underline{ }|}(\mathbb{Q} / \mathbb{Z}) & \longrightarrow \mathrm{M}_{|\underline{ }|}(\mathbb{Q} / \mathbb{Z}), & \mathcal{P}^{* \wedge 2}: \operatorname{Alt}_{|\underline{ }|}(\mathbb{Q} / \mathbb{Z}) & \longrightarrow \operatorname{Alt}_{|\underline{ }|}(\mathbb{Q} / \mathbb{Z}), \\
\left(b_{i j}\right) & \longmapsto\left(b_{i j}^{\prime}\right), & \left(a_{i j}\right) & \longmapsto\left(a_{i j}^{\prime}\right),
\end{aligned}
$$

where $b_{i j}^{\prime}=\sum_{k, l} p_{i k}^{*} p_{j l}^{*} b_{k l}$ and $a_{i j}^{\prime}=\sum_{k<l} p_{i j, k l}^{*} a_{k l}$, where $p_{i j}^{*}=p_{j i}$, and $p_{k l, i j}^{*}=$ $p_{k i}^{*} p_{l j}^{*}-p_{l i}^{*} p_{k j}^{*}$.

(c) Suppose $\iota$ is open. Let $s=[G: H]$ and $\mathcal{N}=\left(n_{i j}\right)=[j] \frac{\psi}{\phi}$. The coordinate expression of the corestriction map is

$$
\begin{aligned}
\mathcal{N}^{* \wedge 2} \cdot \frac{1}{s}: \operatorname{Alt}_{|\underline{\mid}|}(\mathbb{Q} / \mathbb{Z}) & \longrightarrow \operatorname{Alt}_{|\underline{\phi}|}(\mathbb{Q} / \mathbb{Z}) \\
\left(a_{i j}\right) & \longmapsto\left(a_{i j}^{\prime}\right)
\end{aligned}
$$

defined by $a_{i j}^{\prime}=\sum_{k<l} n_{i j, k l}^{*} \frac{1}{s} a_{k l}$, where $n_{i j}^{*}=n_{j i}, n_{i j, k l}^{*}=n_{i k}^{*} n_{j l}^{*}-n_{i l}^{*} n_{j k}^{*}$, and $\frac{1}{s} a_{i j}$ is any element in $\mathbb{Q} / \mathbb{Z}$ such that $s \frac{1}{s} a_{i j}=a_{i j}$.

Proof. Let $\mathcal{B P C}=\mathcal{D}=\operatorname{diag}\left\{d_{i}\right\}_{I}$ be the diagonalization of Lemma 6.3. Then $\mathcal{C}^{*} \mathcal{P}^{*} \mathcal{B}^{*}=\mathcal{D}^{*}=\operatorname{diag}\left\{d_{i}\right\}_{I}$, and both $\underline{\phi}^{* \prime}=\underline{\phi}^{*} \mathcal{B}^{*}$ and $\underline{\psi}^{* \prime}=\underline{\psi}^{*}\left(\mathcal{C}^{*}\right)^{-1}$ are (families of) basis changes for $G^{*}$ and $H^{*}$, respectively, and $\mathcal{D}^{*}=\left[\iota^{*}\right]_{\frac{\phi^{* \prime}}{\psi^{* \prime}}}$. By Proposition 5.4, $\phi^{* \otimes 2}, \psi^{* \prime \otimes 2}, \underline{\phi}^{* \prime \wedge 2}$, and $\psi^{* \prime \wedge 2}$ are (families of) bases for $\operatorname{Bil}(G, \mathbb{Q} / \mathbb{Z}), \operatorname{Bil}(H, \mathbb{Q} / \mathbb{Z})$, $\overline{\operatorname{Alt}}(G, \overline{\mathbb{Q}} / \mathbb{Z})$, and $\operatorname{Alt}(\bar{H}, \mathbb{Q} / \mathbb{Z})$, respectively. Compute by the formulas in Theorem 2.4, $\left[\iota^{* \otimes 2}\right] \frac{\psi^{* \prime}}{\phi^{* \prime}}=\mathcal{D}^{* \otimes 2}=\operatorname{diag}\left\{d_{i j}\right\}_{i, j}$ and $\left[\iota^{* \wedge 2}\right] \frac{\psi^{* \prime}}{\phi^{* \prime}}=\mathcal{D}^{* \wedge 2}=\operatorname{diag}\left\{d_{i j}\right\}_{i<j}$. Thus res $\left(\phi_{i}^{* \prime} \otimes \phi_{j}^{* \prime}\right)_{d}=d_{i j}\left(\psi_{i}^{* \prime} \otimes \psi_{j}^{* \prime}\right)_{d}$ and $\operatorname{res}\left(\phi_{i}^{* \prime} \wedge \bar{\phi}_{j}^{* \prime}\right)_{d}=d_{i j}\left(\psi_{i}^{* \prime} \wedge \psi_{j}^{* \prime}\right)_{d}$. Since $\phi^{* \prime \otimes 2}$ and $\phi^{* \prime \wedge 2}$ are bases, it follows that the kernels are the subgroups with bases

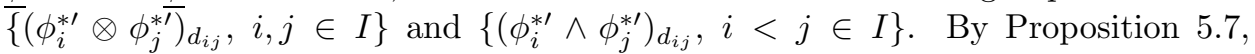
$\left(\phi_{i}^{* \prime} \otimes \phi_{j}^{* \prime}\right)_{d_{i j}}=\sum_{k, l} b_{k i}^{*} b_{l j}^{*}\left(\phi_{k}^{*} \otimes \phi_{l}^{*}\right)_{d_{i j}}$ and $\left(\phi_{i}^{* \prime} \wedge \phi_{j}^{* \prime}\right)_{d_{i j}}=\sum_{k<l} b_{k l, i j}^{*}\left(\phi_{k}^{*} \wedge \phi_{l}^{*}\right)_{d_{i j}}$, so the kernels of the restrictions are as stated. This proves (a).

For (b), view $H$ as a subgroup of $G$, which is allowed since $\iota$ is closed. Since $\mathcal{P}=[\iota]_{\underline{\underline{\psi}}}^{\underline{\phi}}, \mathcal{P}^{*}=\left[\iota^{*}\right]_{\underline{\phi^{*}}}$. A computation exactly as in Proposition 5.7 for basis change gives the desired formulas for the coordinate expressions $\mathcal{P} * \otimes 2=\left[\iota^{* \otimes 2}\right]{\underline{\underline{\phi^{*}}}}^{*}$ and $\mathcal{P}^{* \wedge 2}=\left[\iota^{* \wedge 2}\right] \frac{\psi^{*}}{\underline{\phi}^{*}}$.

Suppose $H \leq G$ is an open subgroup, and $[G: H]=s$. By Theorem 2.10, $\operatorname{cor}(\boldsymbol{a})=\jmath^{* \wedge 2}\left(\frac{1}{s} \boldsymbol{a}\right)$ on $\operatorname{Alt}(H, \mathbb{Q} / \mathbb{Z})$, where $\frac{1}{s} \boldsymbol{a}$ is an $s$-th root of $\boldsymbol{a}$. Clearly if $[\boldsymbol{a}]_{\underline{\psi}}=\left(a_{i j}\right)$, then $\left[\frac{1}{s} \boldsymbol{a}\right]_{\underline{\psi}}=\left(\frac{1}{s} a_{i j}\right)$, where $\frac{1}{s} a_{i j}$ is any $s$-th root of $a_{i j}$ in $\mathbb{Z}[1 / \mathfrak{m}] / \mathbb{Z}$. If $\mathcal{N}=[]]_{\underline{\phi}}^{\frac{\psi}{\phi}}=\left(n_{i j}\right)$, the transpose is $\mathcal{N}^{*}=\left[\jmath^{*}\right] \frac{\phi^{*}}{\underline{\psi}^{*}}=\left(n_{i j}^{*}\right)$, and

$$
\mathcal{N}^{* \wedge 2}=\left[j^{* \wedge 2}\right]{\underline{\phi^{*}}}^{*}:\left[\frac{1}{s} \boldsymbol{a}\right]_{\underline{\underline{ }}} \longmapsto[\operatorname{cor}(\boldsymbol{a})]_{\underline{\phi}} .
$$

If $[\operatorname{cor}(\boldsymbol{a})]_{\underline{\phi}}=\left(a_{i j}^{\prime}\right)$, then a routine computation shows $a_{i j}^{\prime}=\sum_{k<l} n_{i j, k l}^{*} \frac{1}{s} a_{k l}$.

Example 6.5. Suppose $G=\widehat{\mathbb{Z}}^{n}$. Let $H \leq G$ be a subgroup of index $s$. Let $\iota: H \hookrightarrow$ $G$ be the natural inclusion. Suppose $\phi=\left\{\phi_{1}, \ldots, \phi_{n}\right\}$ and $\psi=\left\{\psi_{1}, \ldots, \psi_{n}\right\}$ are bases. The restriction and corestriction are computed on $\operatorname{Alt}_{n}(\mathbb{Q} / \mathbb{Z})$ from Theorem 6.4 as follows. 


\section{Restriction.}

Let $\mathcal{P}^{*}=\left[\iota^{*}\right] \frac{\psi^{*}}{\phi^{*}} ;$ then $\mathcal{P}^{*}=\left(p_{i j}^{*}\right) \in \mathrm{M}_{n}(\widehat{\mathbb{Z}})$ has $\operatorname{rank} n$. If $\boldsymbol{a} \in \operatorname{Alt}(G, \mathbb{Q} / \mathbb{Z})$, then $[\boldsymbol{a}]_{\phi}=\left(a_{i j}\right) \in \operatorname{Alt}_{n}(\mathbb{Q} / \mathbb{Z})$, where $a_{i j}=\boldsymbol{a}\left(\phi_{i}, \phi_{j}\right)$. Set $\operatorname{res}(\boldsymbol{a})=\boldsymbol{a}^{\prime}$ and $\left[\boldsymbol{a}^{\prime}\right]_{\underline{\psi}}=\left(a_{i j}^{\prime}\right) \in \operatorname{Alt}_{n}(\mathbb{Q} / \mathbb{Z})$. As in Example 5.8, order the pairs $i j$ lexicographically, and view $\left(a_{i j}\right)$ as the $\left(\begin{array}{c}n \\ 2\end{array}\right)$-tuple $\left(a_{i j}\right)=\left\{a_{12}, a_{13}, \ldots, a_{n-1 n}\right\}$. By Theorem 6.4, $[\operatorname{res}]]_{\underline{\phi}^{*}}=\mathcal{P}^{* \wedge}=\left(p_{k l, i j}^{*}\right) \in \mathrm{M}_{\left(\begin{array}{c}n \\ 2\end{array}\right)}(\widehat{\mathbb{Z}})$, where $p_{k l, i j}^{*}=p_{k i}^{*} p_{l j}^{*}-p_{l i}^{*} p_{k j}^{*}$ is the entry in the $k l$-th row and $i j$-th column. Compute $\mathcal{P}^{* \wedge 2}\left(a_{i j}\right)=\left(a_{i j}^{\prime}\right)$, where $a_{i j}^{\prime}=\sum_{k<l} p_{i j, k l}^{*} a_{k l}$.

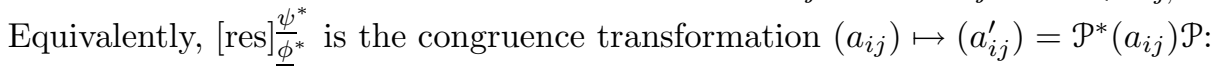

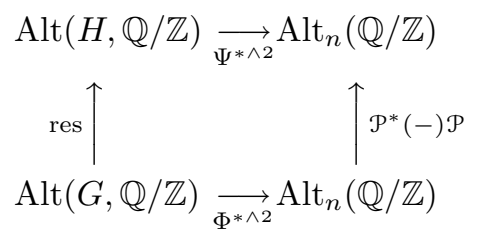

Here $\Psi^{* \wedge 2}$ and $\Phi^{* \wedge 2}$ are the coordinate representation maps with respect to $\underline{\psi}$ and $\phi$, respectively. Let $\mathcal{D}^{*}=\operatorname{diag}\left\{d_{1}, \ldots, d_{n}\right\}$ be a diagonalization of $\mathcal{P}^{*}$, as in Lemma $\overline{6} .3$, with $d_{i} \in \mathbb{N}$. Clearly $\prod d_{i}=[G: H]$, consequently $\operatorname{det}\left(\mathcal{P}^{*}\right)=u[G: H]$, where $u \in \widehat{\mathbb{Z}}^{\times}$and $\operatorname{det}\left(\mathcal{P}^{*}\right)$ is computed in the ring $\widehat{\mathbb{Z}}$. Thus

$$
\left|\operatorname{det}\left(\mathcal{P}^{*}\right)\right|=[G: H]
$$

where $|\cdot|$ is the canonical norm on $\widehat{\mathbb{Z}}$.

In the special case $n=2, \mathcal{P}^{* \wedge 2}=p_{12,12}^{*}=p_{11}^{*} p_{22}^{*}-p_{12}^{*} p_{21}^{*}=\operatorname{det}\left(\mathcal{P}^{*}\right)$, so restriction is multiplication by $u[G: H]$ :

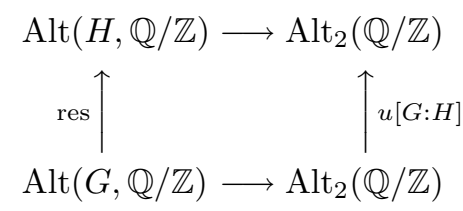

\section{Corestriction.}

Since $\operatorname{rank}\left(\mathcal{P}^{*}\right)=n, \mathcal{P}^{*} \in \mathrm{GL}_{n}(\widehat{\mathbb{Q}})$. Since $\mathcal{P}^{*} \mathcal{N}^{*}$ is multiplication by $s, \mathcal{N}^{*}=$ $s\left(\mathcal{P}^{*}\right)^{-1}$. Let $\mathbb{Q}^{*}=\left(\mathcal{P}^{*}\right)^{-1} \in \mathrm{GL}_{n}(\widehat{\mathbb{Q}})$, and define the action of $\mathbb{Q}^{*}=\frac{1}{s} \mathcal{N}^{*}$ on $\mathbb{Q} / \mathbb{Z}$ by letting $\frac{1}{s}$ produce an $s$-th root of an element of $\mathbb{Q} / \mathbb{Z}$. By Theorem 6.4

$$
[\operatorname{cor}] \underline{\underline{\phi}}^{\underline{\phi}^{*}}=s Q^{* \wedge 2}=\left(s q_{k l, i j}^{*}\right) \quad \in \mathrm{GL}_{n}(\widehat{\mathbb{Q}}),
$$

where $q_{k l, i j}^{*}=q_{k i}^{*} q_{l j}^{*}-q_{l i}^{*} q_{k j}^{*}$. Write $[\boldsymbol{b}]_{\underline{\psi}}=\left(b_{i j}\right)$; then $[\operatorname{cor}(\boldsymbol{b})]_{\underline{\phi}}=s Q^{* \wedge 2}\left(b_{i j}\right)=\left(b_{i j}^{\prime}\right)$, where $b_{i j}^{\prime}=\sum_{k<l} s q_{i j, k l}^{*} b_{k l}=\sum_{k<l} n_{i j, k l}^{*} \frac{1}{s} b_{k l}$. By abuse of notation, [cor $]_{\underline{\psi}^{*}}^{\frac{\phi^{*}}{i j}}$ is a 
congruence transformation:

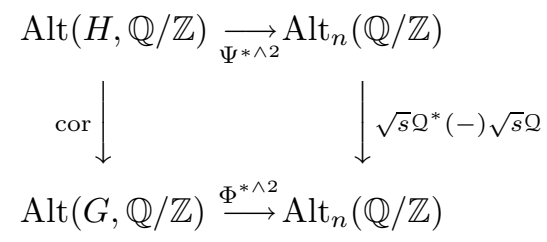

In the special case $n=2$, the corestriction is multiplication by $v$, where $v=$ $u^{-1} \in \widehat{\mathbb{Z}}^{\times}$, as above, which is an isomorphism. Thus

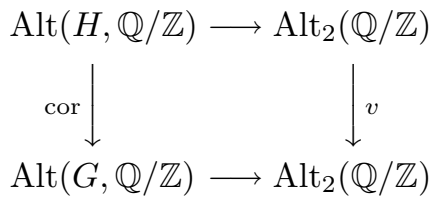

Computing Functorial Maps in $\operatorname{Br}(F)$

Setup 6.6. The following situation holds for the rest of the section. Let $F$ be a field that contains the roots of unity $\mu$. Let $\omega: \mathbb{Q} / \mathbb{Z} \rightarrow \mu$ denote a fixed isomorphism. Let

$$
1 \longrightarrow \mathrm{G}_{\Omega / K} \stackrel{\iota}{\longrightarrow} \mathrm{G}_{\Omega / F} \stackrel{\pi}{\longrightarrow} \mathrm{G}_{K / F} \longrightarrow 1
$$

be a short exact sequence of abelian groups, where $\mathrm{G}_{\Omega / F}$ is torsion-free, and $\exp \left(\mathrm{G}_{K / F}\right)=r \in \mathbb{N}$. Assume $r>1$. For $d: r \mid d$, let

$$
\jmath_{d}: \mathrm{G}_{\Omega / F} \rightarrow \mathrm{G}_{\Omega / K}
$$

denote exponentiation by $d$. If $\iota$ is open, set $s=\left|\mathrm{G}_{K / F}\right|$, and let $\jmath:=\jmath_{s}$ denote the transfer. Let

$$
\kappa_{\omega_{d}}: \mathrm{G}_{\Omega / F}^{*} \stackrel{\sim}{\longrightarrow}\left(\Omega^{d} \cap F^{\times}\right) / F^{\times d}
$$

denote the Kummer isomorphism of Setup 4.1.

Lemma 6.7. Suppose $\underline{\underline{\psi}}$ and $\underline{\phi}$ are compatible topological bases for $\mathrm{G}_{\Omega / K}$ and $\mathrm{G}_{\Omega / F}$, respectively, and $\exp \left(\mathrm{G}_{K / F}\right)=r$. Then for all $d: r \mid d, d \underline{z}:=\kappa_{\omega_{d}} \cdot J_{d}^{*}\left(d \psi^{*}\right)$ generates the Kummer subgroup $K^{d} \cap F^{\times} / F^{\times}$of $F^{\times} / F^{\times} d$ determined by $K / \bar{F}$. If $\underline{z} \subset F^{\times}$ is any preimage of $d \underline{z}$, and $\underline{y}=\underline{z}^{1 / d} \subset K^{\times}$, then $K=F(\underline{y})$.

Proof. Since $\mathrm{G}_{\Omega / F}$ is torsion-free, this is immediate by Lemma 2.7 and the comments preceding Lemma 4.2 .

Suppose $F=\mathbb{C}\left(x_{1}, \ldots, x_{n}\right)$ is the rational function field in $n$-variables over $\mathbb{C}$. Every abelian extension of $F$ is Kummer. A conspicuous class of Kummer extensions are those extensions $K / F$ that can be defined by choosing $n$ radical elements $y_{j}:=\prod x_{i}^{q_{j i}}$, where each $q_{j i}$ is a rational number between 0 and 1 (inclusive), and adjoining these elements to $F$. For this purpose, the $x_{i}$ could easily be replaced by a pure independent subset of $F^{\times}$, such as a set of primes in $\mathbb{C}\left[x_{1}, \ldots, x_{n}\right]$. Let $X$ be the subgroup of $F^{\times}$generated by the $x_{i}$. Each Kummer extension defined in this way may be represented by an $n \times n$ matrix $\mathcal{Q}$ with entries $q_{j i}$ in $\mathbb{Q}$, whose $j$-th 
row consists of the exponents that define $y_{j}$ in terms of the $x_{i}$. For example, the extension $F\left(\left(x_{1} x_{2}\right)^{1 / 4}, x_{2}^{1 / 2}\right)$ of $F=\mathbb{C}\left(x_{1}, x_{2}, x_{3}\right)$ is represented by the matrix

$$
\mathcal{Q}=\left(\begin{array}{ccc}
\frac{1}{4} & \frac{1}{4} & 0 \\
0 & \frac{1}{2} & 0 \\
0 & 0 & 1
\end{array}\right),
$$

so that $y_{1}=\left(x_{1} x_{2}\right)^{1 / 4}, y_{2}=x_{2}^{1 / 2}$, and $y_{3}=x_{3}$. This matrix is invertible, and the rows of the inverse $\mathcal{P}$ express the $x_{i}$ in terms of the $y_{j}$. In this case,

$$
\mathcal{P}=Q^{-1}=\left(\begin{array}{ccc}
4 & -2 & 0 \\
0 & 2 & 0 \\
0 & 0 & 1
\end{array}\right)
$$

so that $x_{1}=y_{1}^{4} y_{2}^{-2}, x_{2}=y_{2}^{2}$, and $x_{3}=y_{3}$. The $y_{j}$ generate a subgroup $Y$ of $K^{\times}$, and the image of $Y$ in $F_{\text {alg }}^{\times} / F^{\times}$is canonically isomorphic to the dual $\mathrm{G}_{K / F}^{*}$ of the Galois group $\mathrm{G}_{K / F}$. The group in the above example has exponent 4 , and so $Y^{4}$ is a subgroup $Z$ of $X$, with generators $z_{i}:=y_{i}^{4}$. This subgroup contains $X^{4}$; more generally $X^{d} \leq Z=Y^{d} \leq X$, where $d=\exp \left(\mathrm{G}_{K / F}\right)$. Obviously $z_{j}=\prod x_{i}^{n_{j i}}$, where $n_{j i}=d q_{j i}=4 q_{j i}$, and the rows of the matrix

$$
\mathcal{N}_{4}=4 Q=\left(\begin{array}{lll}
1 & 1 & 0 \\
0 & 2 & 0 \\
0 & 0 & 4
\end{array}\right)
$$

express the $z_{j}$ in terms of the $x_{i}$. Thus the elements $z_{1}=x_{1} x_{2}, z_{2}=x_{2}^{2}$, and $z_{3}=x_{3}^{4}$ determine the subgroup $Z$. By Kummer theory, the image of $Z$ in $F^{\times} / F^{\times 4}$ is also isomorphic to $\mathrm{G}_{K / F}^{*}$.

Since the machinery developed in Theorem 6.4 to compute restriction and corestriction is phrased in terms of bases of Galois groups and their character groups, it is not obvious how it would apply to such a Kummer extension, given not by a subgroup of the Galois group, but purely in terms of field elements. The next result shows how the subgroups $X, Y$, and $Z$ can be used to reconcile the two points of view. It will allow restriction and corestriction on the Brauer group to be computed (in Theorem 6.11) in the likely case when the starting data is a Kummer extension that is defined explicitly in terms of field elements.

Lemma 6.8. Fix $\mathfrak{m} \subset \mathcal{P}$ and a number $d \in \mathbb{N}(\mathfrak{m})$. Let $\underline{x}=\left\{x_{1}, \ldots, x_{n}\right\}$ be an $\mathfrak{m}$-independent subset of $F^{\times}$, let $\underline{y}=\left\{y_{1}, \ldots, y_{n}\right\}$ be a subset of $F_{\text {alg }}^{\times}$, and let $\underline{z}=\left\{z_{1}, \ldots, z_{n}\right\}$ be defined by $z_{i}=y_{i}^{d}$. Let $X=\langle\underline{x}\rangle, Y=\langle\underline{y}\rangle$, and $Z=\langle\underline{z}\rangle$. Assume $X^{d} \leq Z \leq X$. Set $K=F(Y) \subset \Omega=F\left(X^{1 / \mathfrak{m}^{\infty}}\right)$, so that (6.6.1) and (6.6.2) hold. Then

(a) The subset $\underline{y}$ is $\mathfrak{m}$-independent in $K^{\times}$, and there are isomorphisms ${ }_{d} \mathrm{G}_{\Omega / F}^{*} \simeq$ $X / X^{d},{ }_{d} \mathrm{G}_{\Omega / K}^{*} \simeq Y / Y^{d}$ and ${ }_{d} \mathrm{G}_{K / F}^{*} \simeq Z / X^{d}$, given by $\kappa_{\omega_{d}}$ over the appropriate field.

(b) The natural maps $\pi^{*}: Z \hookrightarrow X, \iota^{*}: X \hookrightarrow Y$, and $\jmath_{d}^{*}: Y \hookrightarrow \pi^{*}(Z)$ induce their counterparts for the groups ${ }_{d} \mathrm{G}_{K / F}^{*},{ }_{d} \mathrm{G}_{\Omega / F}^{*}$, and ${ }_{d} \mathrm{G}_{\Omega / K}^{*}$, and transpose to $\pi$, $\iota$, and $\jmath_{d}$ in (6.6.1) and (6.6.2).

(c) There exist topological bases $\psi=\left\{\psi_{1}, \ldots, \psi_{n}\right\}$ and $\phi=\left\{\phi_{1}, \ldots, \phi_{n}\right\}$ for $\mathrm{G}_{\Omega / K}$ and $\mathrm{G}_{\Omega / F}$, respectively, whose Kummer duals in $K^{\times} / \bar{K}^{\times} e$ and $F^{\times} / F^{\times e}$ are the images of $\underline{x}$ and $\underline{y}$, for all $e \in \mathbb{N}(\mathfrak{m})$. Thus $\left\langle\phi_{i}, x_{j}\right\rangle_{e}=\omega_{e}^{\delta_{i j}}$ and $\left\langle\psi_{i}, y_{j}\right\rangle_{e}=\omega_{e}^{\delta_{i j}}$. 
In particular, the maps $[\iota]_{\frac{\phi}{\psi}}^{\frac{\phi}{\alpha}}=\mathcal{P}=\left(p_{i j}\right)$ and $\left[\jmath_{d}\right]_{\frac{\psi}{\phi}}^{\frac{\psi}{\alpha}}=\mathcal{N}_{d}=\left(n_{i j}\right)$ are the transposes

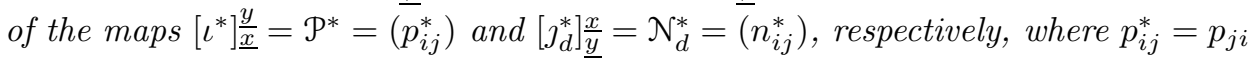
and $n_{i j}^{*}=n_{j i}$ are integers. In other words, if

$$
x_{j}=\prod_{i=1}^{n} y_{i}^{p_{i j}^{*}}, \quad z_{j}=y_{j}^{d}=\prod_{i=1}^{n} x_{i}^{n_{i j}^{*}},
$$

then

$$
\psi_{j}=\prod_{i=1}^{n} \phi_{i}^{p_{i j}}, \quad \phi_{j}^{d}=\prod_{i=1}^{n} \psi_{i}^{n_{i j}} .
$$

(d) $[K: F]=|\operatorname{det}(\mathcal{P})|$, where $\mathcal{P}=[\iota] \frac{\phi}{\underline{\psi}}$.

Proof. Since $\underline{x}$ is m-independent in $F^{\times}, \underline{x}$ is independent in $F^{\times} / F^{\times} d$. By $[\mathrm{F}$, Lemma 32.1], $X$ is m-pure in $F^{\times}$, hence $F^{d} \cap X=X^{d}$. By [F, Proposition 27.1], $X / X^{d} \hookrightarrow$ $F^{\times} / F^{\times d}$ is a summand. Therefore by Definition 5.15 of $\Omega$ as a Kummer extension, $\kappa_{\omega_{d}}:{ }_{d} \mathrm{G}_{\Omega / F}^{*} \stackrel{\sim}{\longrightarrow} X / X^{d}$.

By definition, the extension $K / F$ is determined by the image $Z / Z \cap F^{d}$ of $Z$ in $F^{\times} / F^{\times d}$. Since $X \cap F^{d}=X^{d} \leq Z \leq X, Z / Z \cap F^{d}=Z / X^{d}$. Thus by Kummer theory, $\kappa_{\omega_{d}}: \mathrm{G}_{K / F}^{*} \stackrel{\sim}{\longrightarrow} Z / X^{d}$.

Since $\underline{x}$ is $\mathfrak{m}$-independent in $F^{\times}$and $\mu$ is divisible, $X$ is torsion-free. Since $Z \leq X, Z$ is torsion-free. Since $X^{d} \leq Z, \operatorname{rank}(X)=\operatorname{rank}(Z)$, so $X \simeq Z$, and $\underline{z}$ is a basis for $Z$. The exponentiation-by- $d$ homomorphism $\jmath_{d}^{*}$ on $K^{\times}$maps $Y$ onto $Z$. The kernel $\mu_{d} \cap Y$ is trivial, for if $\prod y_{i}^{u_{i}} \in \mu_{d}$, then $\prod z_{i}^{u_{i}}=1$, which implies $u_{i}=0$ since the $z_{i}$ are independent. Therefore $Y \simeq Z$, and $Y$ is torsion-free, with basis $y$. To show $y$ is $\mathfrak{m}$-independent in $K^{\times}$, it suffices by [F, Lemma 32.1] to show $Y \cap \bar{K}^{p}=Y^{p}$ for all $p \in \mathfrak{m}$. The inclusion $Y \cap K^{p} \geq Y^{p}$ is obvious, so it suffices to show $Y \cap K^{p} \leq Y^{p}$.

Since $Y^{d}=Z \leq X \leq F^{\times}$, by Kummer theory, $\left(K^{\times} / F^{\times}\right)_{\text {tor }}=Y F^{\times} / F^{\times}$. Suppose $p \in \mathfrak{m}$, and $a \in Y \cap K^{p}$. Then $a=y^{p}$ for some $y \in K^{\times}$. Since $a$ is in $Y$, its image in $K^{\times} / F^{\times}$has finite order, and since $a=y^{p}$, the image of $y$ in $K^{\times} / F^{\times}$ also has finite order, so it lies in $\left(K^{\times} / F^{\times}\right)_{\text {tor }}=Y F^{\times} / F^{\times}$. Thus $y=y^{\prime} x$ for some $y^{\prime} \in Y$ and $x \in F^{\times}$, hence $a \in Y^{p} F^{\times} p$. Therefore $Y \cap K^{p} \leq Y^{p} F^{\times p} \cap Y$. Since $Y \cap K^{p} \geq Y^{p} F^{\times p} \cap Y$ is clear, $Y \cap K^{p}=Y^{p} F^{\times p}$. A quick calculation shows $Y^{p} F^{\times}=\left(Y \cap F^{p}\right) Y^{p}$, so $Y \cap K^{p}=\left(Y \cap F^{p}\right) Y^{p}$. It now suffices to show $Y \cap F^{p} \leq Y^{p}$.

Let "-" denote the map $K^{\times} \rightarrow K^{\times} / \mu$. If $\bar{Y} \cap \bar{F}^{p} \leq \bar{Y}^{p}$, then $Y \cap F^{p} \leq Y^{p} \mu \cap Y=$ $Y^{p}(\mu \cap Y)=Y^{p}$, as $Y$ is torsion-free. Therefore it suffices to show $\bar{Y} \cap \bar{F}^{p} \leq \bar{Y}^{p}$. Note that since $\bar{X}^{d} \leq \bar{Y}^{d}$ and $\bar{K}$ is torsion-free, $\bar{X} \leq \bar{Y}$. Also $\bar{X} \cap \bar{F}^{d}=\bar{X}^{d}$, since $X \cap F^{d}=X^{d}$, and there is a commutative diagram

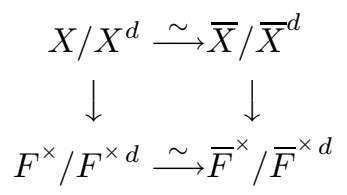

Thus

$$
(\bar{Y} \cap \bar{F})^{d} \leq \bar{Y}^{d} \cap \bar{F}^{d} \leq \bar{X} \cap \bar{F}^{d}=\bar{X}^{d} .
$$

Therefore $\bar{Y} \cap \bar{F} \leq \bar{X}$, as $\bar{K}$ is torsion-free. Therefore $\bar{Y} \cap \bar{F}^{p} \leq \bar{X} \cap \bar{F}^{p}=\bar{X}^{p} \leq \bar{Y}^{p}$, as desired. Thus $\underline{y}$ is $\mathfrak{m}$-independent in $K^{\times}$. 
Since $\underline{y}$ is $\mathfrak{m}$-independent in $K^{\times}$, a proof similar to the one for $\underline{x}$ in $F^{\times}$in the first paragraph shows $\kappa_{\omega_{d}}:{ }_{d} \mathrm{G}_{\Omega / K}^{*} \stackrel{\sim}{\longrightarrow} Y / Y^{d}$. This completes the proof of (a).

The inclusions $\pi^{*}: Z \hookrightarrow X$ and $\iota^{*}: X \hookrightarrow Y$ clearly induce the inflation ${ }_{d} \mathrm{G}_{K / F}^{*} \hookrightarrow{ }_{d} \mathrm{G}_{\Omega / F}^{*}$ and the restriction ${ }_{d} \mathrm{G}_{\Omega / F}^{*} \rightarrow{ }_{d} \mathrm{G}_{\Omega / K}^{*}$. If $\sigma \in \mathrm{G}_{\Omega / F}$ and $y \in$ $Y$, then $\left\langle\sigma, \jmath_{d}^{*}(y)\right\rangle_{d}=\left\langle\sigma, y^{d}\right\rangle_{d}=\left\langle\sigma^{d}, y\right\rangle_{d}=\left\langle\jmath_{d}(\sigma), y\right\rangle_{d}=\jmath_{d}^{*}\left(\langle-, y\rangle_{d}\right)(\sigma)$, using the bilinearity of the Kummer symbol. Therefore the exponentiation-by- $d$ map $\jmath_{d}^{*}: Y \hookrightarrow \pi^{*}(Z)$ induces $\jmath_{d}^{*}:{ }_{d} \mathrm{G}_{\Omega / K}^{*} \rightarrow \mathrm{G}_{\Omega / F}^{*}$. This proves (b).

Suppose $e \in \mathbb{N}(\mathfrak{m})$. The images of $\underline{x}$ and $\underline{y}$ in $X / X^{e}$ and $Y / Y^{e}$ are clearly coherent under the natural projections, since, after all, $\underline{x} \subset F^{\times}$and $y \subset K^{\times}$. Therefore by Lemma 5.14, the desired bases $\phi$ and $\psi$ for $\mathrm{G}_{\Omega / F}$ and $\mathrm{G}_{\Omega / K}$ exist, Kummer dual to the images of $\underline{x}$ and $y$ in $X / \bar{X}^{e}$ and $Y / Y^{e}$, respectively. By (b), the maps on $X / X^{e}$ and $Y / Y^{e}$ induced by $\iota^{*}: X \rightarrow Y$ and $\jmath_{d}^{*}: Y \rightarrow X$ are transpose to $\iota$ and $\jmath_{d}$. Thus if $[\iota]_{\frac{\phi}{\psi}}^{\frac{\phi}{\alpha}}=\mathcal{P}=\left(p_{i j}\right)$ and $\left[\jmath_{d}\right] \frac{\psi}{\phi}=\mathcal{N}_{d}=\left(n_{i j}\right)$, then $\left[\iota^{*}\right] \underline{y} \underline{\underline{y}}=\mathcal{P}^{*}=\left(p_{i j}^{*}\right)$ and $\left[\jmath_{d}^{*}\right] \underline{\underline{x}}=\mathcal{N}_{d}^{*}=\left(n_{i j}^{*}\right)$, where $p_{i j}^{*}=p_{j i}$ and $n_{i j}^{*}=n_{j i}$ are integers. This proves (c).

Finally, the matrix $\left[j_{d}^{*}\right] \underline{\underline{y}}$ expresses each $y_{j}^{d}=z_{j}$ as a product of $x_{i}$ 's. Since $X$ and $Z$ are free abelian groups, it follows by [Ro, Chapter 9, Corollary 9.63] that $\left|\operatorname{det}\left(\left[J_{d}^{*}\right] \underline{\underline{y}}\right)\right|=\left|\operatorname{det}\left(\mathcal{N}_{d}^{*}\right)\right|=[X: Z]$. Similarly, since $\jmath_{d}^{*} \cdot \iota^{*}$ is exponentiation by $d$ on $X$,

$$
\left|\operatorname{det}\left(\left[J_{d}^{*} \cdot \iota^{*}\right] \underline{\underline{x}}\right)\right|=\left|\operatorname{det}\left(\mathcal{N}_{d}^{*} \cdot \mathcal{P}^{*}\right)\right|=[X: Z]\left|\operatorname{det}\left(\mathcal{P}^{*}\right)\right|=\left[X: X^{d}\right]=d^{n} .
$$

Therefore $\left[Z: X^{d}\right]=\left|\operatorname{det}\left(\mathcal{P}^{*}\right)\right|$. Since $K / F$ is defined by the image of $Z$ in $X / X^{d}$, $\left[Z: X^{d}\right]=[K: F]$, hence $[K: F]=\left|\operatorname{det}\left(\mathcal{P}^{*}\right)\right|$. This proves (d).

Remark 6.9. It is easy to construct situations that satisfy the hypotheses of Lemma 6.8. For example, $F$ could be a rational function field in $n$ variables, or the quotient field of a ring containing $n$ nonassociate prime elements. Let $\underline{x}=\left\{x_{1}, \ldots, x_{n}\right\}$ be either of these sets. Let $\mathcal{P}=\left(p_{i j}\right) \in \mathrm{M}_{n}(\mathbb{Z})$ be any matrix with nonzero determinant $d$. Set $\mathcal{Q}=\left(q_{i j}\right)=\mathcal{P}^{-1} \in \mathrm{GL}_{n}(\mathbb{Q})$, and use this to choose $y=\left\{y_{1}, \ldots, y_{n}\right\}$ by the rule $y_{j}=\prod x_{i}^{q_{j i}}$. Since the adjoint of $\mathcal{P}$ is defined over $\mathbb{Z}, d \bar{q}_{i j} \in \mathbb{Z}$, so $z_{j}=y_{j}^{d} \in X$. Since $\mathcal{P}$ is defined over $\mathbb{Z}, X \leq Y$, therefore $X^{d} \leq Z$. Thus all of the hypotheses of Lemma 6.8 are satisfied.

Example 6.10. Revisit the example immediately preceding Lemma 6.8, with $F=$ $\mathbb{C}\left(x_{1}, x_{2}, x_{3}\right)$. Let $\Omega=F\left(\underline{x}^{1 / \infty}\right)$, so that $\mathrm{G}_{\Omega / F} \simeq \widehat{\mathbb{Z}}^{3}$, and let $K$ be the Kummer extension

$$
K=F\left(\left(x_{1} x_{2}\right)^{1 / 4}, x_{2}^{1 / 2}\right) .
$$

The exponent is 4 , which divides $d=8$. In the notation of Lemma $6.8, y_{1}=$ $\left(x_{1} x_{2}\right)^{1 / 4}, y_{2}=x_{2}^{1 / 2}$, and $y_{3}=x_{3}$. The subgroup $Z=\left\langle z_{1}, z_{2}, z_{3}\right\rangle$ is given by $\underline{z}=\underline{y}^{8}$, so that $z_{1}=y_{1}^{8}=\left(x_{1} x_{2}\right)^{2}, z_{2}=y_{2}^{8}=x_{2}^{4}$, and $z_{3}=y_{3}^{8}=x_{3}^{8}$. In matrix notation, $\underline{z}=\underline{x} \mathcal{N}^{*}$, where

$$
\mathcal{N}^{*}=\mathcal{N}_{8}^{*}=\left(\begin{array}{lll}
2 & 0 & 0 \\
2 & 4 & 0 \\
0 & 0 & 8
\end{array}\right) .
$$

Let $Q^{*}:=\frac{1}{8} \mathcal{N}_{8}^{*}$. Since $\mathcal{P}^{*} \mathcal{N}_{8}^{*}=8 \mathcal{J}, \mathcal{P}^{*}=Q^{*-1}$. Compute

$$
Q^{*}=\left(\begin{array}{ccc}
\frac{1}{4} & 0 & 0 \\
\frac{1}{4} & \frac{1}{2} & 0 \\
0 & 0 & 1
\end{array}\right), \quad \mathcal{P}^{*}=\left(\begin{array}{ccc}
4 & 0 & 0 \\
-2 & 2 & 0 \\
0 & 0 & 1
\end{array}\right) .
$$


Then $K=\mathbb{C}\left(y_{1}, y_{2}, y_{3}\right)$, where $\underline{y}=\underline{x} Q^{*}$, i.e., $y_{1}=\left(x_{1} x_{2}\right)^{1 / 4}, y_{2}=x_{2}^{1 / 2}$, and $y_{3}=x_{3}$. Inversely, $\underline{x}=\underline{y} \mathcal{P}^{*}$, i.e., $x_{1}=y_{1}^{4} y_{2}^{-2}, x_{2}=y_{2}^{2}$, and $x_{3}=y_{3}$.

By Lemma 6.8(d), the degree of $K / F$ is

$$
[K: F]=|\operatorname{det}(\mathcal{P})|=8 .
$$

By Lemma $6.8(\mathrm{c}), \mathcal{P}=[\iota]_{\underline{\psi}}$, the transpose of $\mathcal{P}^{*}$, is the coordinate expression for the embedding $\mathrm{G}_{\Omega / K} \hookrightarrow \mathrm{G}_{\Omega / F}$ with respect to bases $\underline{\psi}=\left\{\psi_{1}, \psi_{2}, \psi_{3}\right\}$ and $\underline{\phi}=\left\{\phi_{1}, \phi_{2}, \phi_{3}\right\}$, whose elements are the generators corresponding to the $y_{j}$ and $\bar{x}_{i}$, respectively, as in Lemma 5.14. Thus $\underline{\psi}=\underline{\phi}$ P i.e., $\psi_{1}=\phi_{1}^{4}, \psi_{2}=\phi_{1}^{-2} \phi_{2}^{2}$, and $\psi_{3}=\phi_{3}$.

Theorem 6.11 "Functoriality in $\operatorname{Br}(\boldsymbol{F})$ ". Assume Setup 6.6. Suppose $\mathrm{G}_{\Omega / F} \simeq$ $\mathbb{Z}_{\mathfrak{m}}^{c}$ is homogeneous, for some $\mathfrak{m} \subset \mathcal{P}$ and cardinal number $c$. Let $\psi=\left\{\psi_{i}\right\}_{I}$ and $\phi=\left\{\phi_{i}\right\}_{I}$ be compatible homogeneous topological bases for $\mathrm{G}_{\Omega / F}$ and $\overline{\mathrm{G}}_{\Omega / K}$, indexed $\bar{b} y$ a linearly ordered set $I$. Let $\underline{x}=\left\{x_{i}\right\}_{I}$ and $\underline{y}=\left\{y_{i}\right\}_{I}$ be respective Kummer dual bases, as in Definition 5.15. Set $\mathcal{P}=\left(p_{i j}\right)=[l] \frac{\phi}{\psi}$, and let $\mathcal{B P C}=\mathcal{D}=\operatorname{diag}\left\{d_{i}\right\}_{I}$ be a diagonalization of $\mathcal{P}$ as in Lemma 6.3 , where $\mathcal{B}=\left(b_{i j}\right)$ and $\mathcal{C}$ are basis changes. Then

(a) $\operatorname{Br}^{\circ}(\Omega / F)=\left\langle\left[\left(x_{i}, x_{j}\right)_{\omega_{e}}\right]: i<j, e \in \mathbb{N}(\mathfrak{m})\right\rangle$.

(b) $\operatorname{Br}^{\circ}(K / F)=\left\langle\sum_{k<l} b_{k l, i j}^{*}\left[\left(x_{k}, x_{l}\right)_{\omega_{d_{i} d_{j}}}\right]: i<j\right\rangle$, where $b_{k l, i j}^{*}=b_{i k} b_{j l}-b_{i l} b_{j k}$.

(c) The restriction homomorphism's coordinate expression

$$
\begin{aligned}
& \mathcal{P}^{* \wedge 2}: \operatorname{Br}^{\circ}(\Omega / F) \longrightarrow \operatorname{Br}^{\circ}(\Omega / K), \\
& {[\boldsymbol{\xi}]_{\underline{x}} \longmapsto[\operatorname{res}(\boldsymbol{\xi})]_{\underline{y}} }
\end{aligned}
$$

is defined explicitly by $\operatorname{res}\left(\left[\left(x_{i}, x_{j}\right)_{\omega_{d}}\right]\right)=\sum_{k<l} p_{k l, i j}^{*}\left[\left(y_{k}, y_{l}\right)_{\omega_{d}}\right]$, where $p_{k l, i j}^{*}=$ $p_{i k} p_{j l}-p_{i l} p_{j k}$.

(d) Suppose $\mathrm{G}_{K / F}$ is finite, of order s. Let $\mathcal{N}=\left(n_{i j}\right)=[j] \frac{\psi}{\underline{\phi}}$, the coordinate expression of the transfer. Then the corestriction's coordinate expression

$$
\begin{array}{r}
\mathcal{N}^{* \wedge 2} \cdot \frac{1}{s}: \operatorname{Br}^{\circ}(\Omega / K) \\
{\left[\boldsymbol{\eta}_{\underline{y}} \longmapsto[\operatorname{cor}(\boldsymbol{\eta})]_{\underline{x}}\right.}
\end{array}
$$

is defined explicitly by $\operatorname{cor}\left(\left[\left(y_{i}, y_{j}\right)_{\omega_{d}}\right]\right)=\sum_{k<l} n_{k l, i j}^{*}\left[\left(x_{k}, x_{l}\right)_{\omega_{d s}}\right]$, where $n_{k l, i j}^{*}=$ $n_{i k} n_{j l}-n_{i l} n_{j k}$.

Proof. Suppose $e \in \mathbb{N}(\mathfrak{m})$. By the definition of $\Omega / F, \Omega^{e} \cap F^{\times} / F^{\times e}$ has Kummer basis elements $e \underline{x}$, so (a) follows from Theorem 4.4. Parts (b), (c), and (d) are immediate from Theorem 6.4(a), (b), (c).

Remark 6.12. The hypothesis on $\mathrm{G}_{\Omega / F}$ holds for $\Omega$ equal to the maximal abelian $p$-extension of $F$, for then $\mathrm{G}_{\Omega / F} \simeq \mathbb{Z}_{p}^{c}$. In this case $\operatorname{Br}^{\circ}(\Omega / F) \simeq \operatorname{Br}(\Omega / F)$. For suppose $\boldsymbol{\xi}=\left[(x, y)_{\omega_{d}}\right]$ for some $p$-power $d$ and elements $x, y \in F^{\times}$. Then $F\left(x^{1 / d}\right)$ and $F\left(y^{1 / d}\right)$ are subfields of $\Omega$, so $x, y \in \Omega^{d} \cap F^{\times}$, hence $\boldsymbol{\xi} \in \operatorname{Br}^{\circ}(\Omega / F)$ by Theorem 4.4. Since $\operatorname{Br}(\Omega / F)$ is generated by symbols of $p$-primary order, it follows that $\operatorname{Br}^{\circ}(\Omega / F)=\operatorname{Br}(\Omega / F)$. 
Example 6.13. Continue Example 6.10. It is easy to diagonalize $\mathcal{P}$ :

$$
\mathcal{D}=\mathcal{B P}=\left(\begin{array}{lll}
1 & 1 & 0 \\
0 & 1 & 0 \\
0 & 0 & 1
\end{array}\right)\left(\begin{array}{ccc}
4 & -2 & 0 \\
0 & 2 & 0 \\
0 & 0 & 1
\end{array}\right)=\left(\begin{array}{lll}
4 & 0 & 0 \\
0 & 2 & 0 \\
0 & 0 & 0
\end{array}\right) .
$$

To compute $\operatorname{Br}^{\circ}(K / F)$ and the coordinate expressions of res and cor, compute

$$
\begin{array}{cc}
\mathcal{B}^{* \wedge 2}=\left(\begin{array}{lll}
1 & 0 & 0 \\
0 & 1 & 0 \\
0 & 1 & 1
\end{array}\right), & \mathcal{P}^{* \wedge 2}=\left(\begin{array}{ccc}
8 & 0 & 0 \\
0 & 4 & 0 \\
0 & -2 & 2
\end{array}\right), \\
\frac{1}{8} \mathcal{N}^{* \wedge 2}=\left(\begin{array}{lll}
1 & 0 & 0 \\
0 & 2 & 0 \\
0 & 2 & 4
\end{array}\right), & \mathcal{D}^{* \wedge 2}=\left(\begin{array}{lll}
8 & 0 & 0 \\
0 & 4 & 0 \\
0 & 0 & 2
\end{array}\right) .
\end{array}
$$

By Theorem 6.11(b), $\operatorname{Br}^{\circ}(K / F)$ is generated by the elements $\left[\left(x_{i}, x_{j}\right)_{\omega_{d_{i} d_{j}}}\right] \mathcal{B}^{* \wedge 2}$, so

$$
\operatorname{Br}^{\circ}(K / F)=\left\langle\left[\left(x_{1}, x_{2}\right)_{\omega_{8}}\right], \quad\left[\left(x_{1} x_{2}, x_{3}\right)_{\omega_{4}}\right], \quad\left[\left(x_{2}, x_{3}\right)_{\omega_{2}}\right]\right\rangle .
$$

The restriction and corestriction on basic classes are given by multiplication by $\mathcal{P}^{* \wedge 2}$ and $\frac{1}{8} \mathcal{N}^{* \wedge 2}$,

$$
\begin{aligned}
& \operatorname{res}\left(\left[\left(x_{1}, x_{2}\right)_{\omega_{d}}\right]\right)=8\left[\left(y_{1}, y_{2}\right)_{\omega_{d}}\right], \\
& \operatorname{res}\left(\left[\left(x_{1}, x_{3}\right)_{\omega_{d}}\right]\right)=4\left[\left(y_{1}, y_{3}\right)_{\omega_{d}}\right]-2\left[\left(y_{2}, y_{3}\right)_{\omega_{d}}\right], \\
& \operatorname{res}\left(\left[\left(x_{2}, x_{3}\right)_{\omega_{d}}\right]\right)=2\left[\left(y_{2}, y_{3}\right)_{\omega_{d}}\right], \\
& \operatorname{cor}\left(\left[\left(y_{1}, y_{2}\right)_{\omega_{d}}\right]\right)=\left[\left(x_{1}, x_{2}\right)_{\omega_{d}}\right], \\
& \operatorname{cor}\left(\left[\left(y_{1}, y_{3}\right)_{\omega_{d}}\right]\right)=2\left[\left(x_{1}, x_{3}\right)_{\omega_{d}}\right]+2\left[\left(x_{2}, x_{3}\right)_{\omega_{d}}\right], \\
& \operatorname{cor}\left(\left[\left(y_{2}, y_{3}\right)_{\omega_{d}}\right]\right)=4\left[\left(x_{2}, x_{3}\right)_{\omega_{d}}\right] .
\end{aligned}
$$

Here $d$ is any number. It is easy to check that cor $\cdot$ res is multiplication by $8=$ $[K: F]$. For example,

$$
\begin{aligned}
\operatorname{cor}\left(\operatorname{res}\left(\left[\left(x_{1}, x_{3}\right)_{\omega_{d}}\right]\right)\right) & =\operatorname{cor}\left(4\left[\left(y_{1}, y_{3}\right)_{\omega_{d}}\right]-2\left[\left(y_{2}, y_{3}\right)_{\omega_{d}}\right]\right) \\
& =4\left(2\left[\left(x_{1}, x_{3}\right)_{\omega_{d}}\right]+2\left[\left(x_{2}, x_{3}\right)_{\omega_{d}}\right]\right)-2\left(4\left[\left(x_{2}, x_{3}\right)_{\omega_{d}}\right]\right) \\
& =8\left[\left(x_{1}, x_{3}\right)_{\omega_{d}}\right] .
\end{aligned}
$$

Here, $\left[\left(x_{1}, x_{3}\right)_{\omega_{d}}\right]$ corresponds to the 3 -tuple $(0,1 / d, 0)$. Apply $\mathcal{P}^{* \wedge 2}$ to obtain $(0,4 / d,-2 / d)$, which corresponds to $4\left[\left(y_{1}, y_{3}\right) \omega_{d}\right]-2\left[\left(y_{2}, y_{3}\right) \omega_{d}\right]$. Apply $8 Q^{* \wedge 2}$ to $(0,4 / d,-2 / d)$, to obtain $(0,8 / d, 8 / d-8 / d)=(0,8 / d, 0)$, which corresponds to $8\left[\left(x_{1}, x_{3}\right)_{\omega_{d}}\right]$, as desired.

\section{Acknowledgement}

The author thanks Adrian Wadsworth for invaluable, detailed feedback on preliminary drafts, including the improvement of several proofs. The author thanks David Saltman and Emily Hamilton for valuable comments and suggestions. The author also thanks the UCLA and UCSD Math Departments for extending their warm hospitality during the preparation of this paper. 


\section{REFERENCES}

[AT] S. A. Amitsur and J.-P. Tignol, Kummer subfields of Malcev-Neumann division algebras, Israel J. Math. 50 (1985), 114-144. MR0788071 (86m:16007)

[B] E. S. Brussel, The division algebras and Brauer group of a strictly henselian field, J. Algebra, 239 (2001), 391-411. MR.1827890(2002b:16027)

[D] P. Draxl, Skew Fields, London Math. Soc. Lecture Note Series, no. 81, Cambridge Univ. Press, Cambridge, 1983. MR0696937 (85a:16022)

[F] L. Fuchs, Infinite Abelian Groups, Volume I, Pure and Applied Mathematics, Volume 36, Academic Press, New York, 1970. MR0255673 (41:333)

[K] I. Kaplansky, Infinite Abelian Groups, The University of Michigan Press, Ann Arbor, 1969. MR0233887 (38:2208)

[L] S. Lang, Algebra, Addison-Wesley, Reading, Massachusetts, 1975. MR0197234 (33:5416)

[N] J. Neukirch, A. Schmidt, and K. Wingberg, Cohomology of Number Fields, Springer, New York, 2000. MR $1737196(2000 \mathrm{j}: 11168)$

[R] I. Reiner, Maximal Orders, Academic Press, London, 1975. MR0393100(52:13910)

[RZ] L. Ribes and P. Zalesskii, Profinite Groups, Springer, New York, 2000. MR1775104 (2001k:20060)

[Ro] J. Rotman, Advanced Modern Algebra, Prentice Hall, New Jersey, 2001. MR2043445 (2005b:00002)

[S] J.-P. Serre, Local Fields, Springer, New York, 1979. MR0554237 (82e:12016)

[TW] J.-P. Tignol and A. R. Wadsworth, Totally ramified valuations on finite-dimensional division algebras, Trans. Amer. Math. Soc., 302 (1987), 223-250. MR0887507(88j:16025)

Department of Mathematics and Computer Science, Emory University, Atlanta, Georgia 30322

E-mail address: brussel@mathcs.emory.edu 